\title{
PARADOXICAL DECOMPOSITIONS, 2-GENERATOR KLEINIAN GROUPS, AND VOLUMES OF HYPERBOLIC 3-MANIFOLDS
}

\author{
Marc Culler $^{1}$ and Peter B. Shalen ${ }^{2}$
}

\section{INTRODUCTION}

The $\epsilon$-thin part of a hyperbolic manifold, for an arbitrary positive number $\epsilon$, is defined to consist of all points through which there pass homotopically non-trivial curves of length at most $\epsilon$. For small enough $\epsilon$, the $\epsilon$-thin part is geometrically very simple: it is a disjoint union of standard neighborhoods of closed geodesics and cusps. (Explicit descriptions of these standard neighborhoods are given in Section 1.) If $\epsilon$ is small enough so that the $\epsilon$-thin part of $M$ has this structure then $\epsilon$ is called a Margulis number of $M$. There is a positive number, called a 3-dimensional Margulis constant, which serves as a Margulis number for every hyperbolic 3-manifold.

The results of this paper provide surprisingly large Margulis numbers for a wide class of hyperbolic 3-manifolds. In particular we obtain the following result, which is stated as Theorem 10.3:

Let $M$ be a closed orientable hyperbolic 3-manifold whose first Betti number is at least 3. Then $\log 3=1.09 \ldots$ is a Margulis number for $M$.

Knowing a Margulis number for a given manifold provides important geometric information. For example, one can give an estimate of the volume of a hyperbolic manifold $M$ in terms of a Margulis number $\epsilon$ for $\mathrm{M}$. One first observes that $M$ must contain a hyperbolic ball of radius $\epsilon / 2$; indeed, any point in the thick part of $M$ is the center of such a ball. The volume of this ball is a lower bound for the volume of $M$. This bound can be improved by using an observation of Meyerhoff's [Me]: the ratio of the volume of $M$ to that of the ball is at least $d(\epsilon / 2)$, where $d(r)$ is an explicitly given function which, by a theorem of Böröczky, is a bound for the local density of a radius $r$ sphere-packing in hyperbolic space. This technique gives the following corollary (10.4) to Theorem 10.3:

Let $M$ be a closed orientable hyperbolic 3-manifold whose first Betti number is at least 3. Then $M$ contains a hyperbolic ball of radius $\frac{1}{2} \log 3=.54 \ldots$ and the volume of $M$ is greater than .92 .

1. Partially supported by the National Science Foundation, the Sloan Foundation and the Mathematical Sciences Research Institute

2.Partially supported by the National Science Foundation 
The estimates given by the above results should be compared with other known estimates and with known examples. The greatest known lower bound for the volume of an arbitrary closed orientable hyperbolic 3-manifold, due to F. Gehring and G. Martin [GeM], is .00115, improving an earlier estimate of .00082 by R. Meyerhoff. The smallest known orientable hyperbolic 3-manifold in terms of volume was discovered by J. Weeks; it has Betti number 0 and has a volume of approximately .94. A result of Shalen and Wagreich [ShW] implies, for a 3-manifold $M$ satisfying somewhat weaker hypotheses than those of Theorem 10.3, that $\frac{1}{2} \log 3$ is a Margulis number for $M$ (so that $M$ contains a hyperbolic ball of radius $\frac{1}{4} \log 3$ ).

It can be proved that $\log 3$ is not a Margulis number for the complement of the figure 8 knot, and hence that it is not a Margulis constant. Furthermore, there is overwhelming evidence, based on computer experiments by C. Hodgson and J. Weeks, that the largest hyperbolic ball in the Weeks manifold has radius $.51 \ldots<\frac{1}{2} \log 3$. Thus Theorem 10.3, and - according to the experimental evidence - Corollary 10.4 as well, become false if one drops the topological restrictions on the manifold.

Theorem 10.3 is an application of a theorem on 2-generator Kleinian groups. We may regard the orientable hyperbolic 3-manifold $M$ as the quotient of the hyperbolic 3-space $\mathbf{H}^{3}$ by a discrete group $\Gamma$ of orientation-preserving isometries; from this point of view, $\epsilon$ is a Margulis number for $M$ if and only if, for any two non-commuting elements $\xi$ and $\eta$ of $\Gamma$, every point of $\mathbf{H}^{3}$ is moved a distance at least $\epsilon$ by either $g$ or $h$.

A 3-manifold is said to be topologically tame if it is homeomorphic to the interior of a compact 3-manifold; a torsion-free Kleinian group $\Gamma$ is topologically tame if $\mathbf{H}^{3} / \Gamma$ is topologically tame. We shall prove the following result, which is a stated as Theorem 9.1:

Let $\xi$ and $\eta$ be non-commuting isometries of $\mathbf{H}^{3}$. Suppose that $\xi$ and $\eta$ generate a torsion-free discrete group which is topologically tame, is not co-compact and contains no parabolics. Then every point of hyperbolic space is moved a distance at least $\log 3$ by either $\xi$ or $\eta$; that is, we have

$$
\max (\operatorname{dist}(z, \xi \cdot z), \operatorname{dist}(z, \eta \cdot z)) \geq \log 3
$$

for any $z \in \mathbf{H}^{3}$.

In Section 10 we show (Proposition 10.2) that if $M=\mathbf{H}^{3} / \Gamma$ has first Betti number at least 3 then every two-generator subgroup of $\Gamma$ is non-co-compact and topologically tame. Thus Theorem 9.1 on two-generator groups implies Theorem 10.3 about Margulis numbers.

It is conjectured that any hyperbolic 3-manifold with finitely generated fundamental group is topologically tame. If this were true it would allow one to generalize the above result about Margulis numbers to any hyperbolic 3-manifold whose fundamental group has no 2-generator subgroup of finite index.

We now describe the ingredients of the proof of our main theorem. The proof begins with the observation (Propositon 9.2) that if $\xi$ and $\eta$ are non-commuting isometries of $\mathbf{H}^{3}$ and if the group which they generate is torsion-free, is not co-compact and contains no parabolics then it is free of rank 2 .

One novel feature of the proof is the use of a construction, due to Patterson and studied extensively by Sullivan, of a geometrically natural measure on the limit set of a Kleinian 
group. We use such a measure to relate the combinatorial structure of the free group of rank 2 to its action on its limit set. This part of the argument bears an intriguing resemblance to the construction of a decomposition of the sphere in the Hausdorff-BanachTarski paradox [Wag]. By viewing the free group of rank 2 as a set of reduced words in the alphabet $\left\{x, y, x^{-1}, y^{-1}\right\}$, one obtains a decomposition of the group into four infinite sets and a singleton $\{1\}$, where each of the infinite sets consists of all words beginning with a given letter. This decomposition has the peculiar property that each of the infinite sets is mapped onto the complement of another of the infinite sets under left multiplication by a certain letter. For example, left multiplication by $x^{-1}$ sends a word which begins with $x$ to a word which does not begin with $x^{-1}$.

In the Hausdorff-Banach-Tarski construction one has a rank-2 free group acting by isometries on the sphere. A choice of a point in a given free orbit determines an identification of the group with the orbit and hence induces a decomposition of the orbit. By choosing a point from each orbit one obtains a decomposition of the sphere (ignoring the countable subset of points with non-trivial stabilizer.) Since in this situation the group action preserves the area measure of the sphere, one obtains a paradoxical conclusion.

In our situation we have a free group of rank 2 acting by isometries on hyperbolic space, and hence acting by Möbius transformations on the sphere at infinity. Using a generalization of Patterson's construction we are able to construct a measure on the limit set of our group which decomposes as a sum of four measures, each of which is transformed to the complement of another by a generator or the inverse of a generator. The key to this construction is the fact that the Patterson measures are obtained as limits of measures which are supported on an orbit of the group, and hence reflect the combinatorial structure of the group itself. It is necessary here to identify the group with an orbit by making a choice of a point $z$ in hyperbolic space.

This construction is especially interesting in the case where the Patterson measure that admits the decomposition is equal to the area measure on the sphere at infinity. (Here the sphere is given the round metric obtained by identifying $\mathbf{H}^{3}$ with a ball so that the given point $z$ is the center, and the area measure is normalized so as to have total mass 1.) In this case the "paradoxical" decomposition leads to an elementary proof of the inequality $\max (\operatorname{dist}(z, \xi \cdot z), \operatorname{dist}(z, \eta \cdot z)) \geq \log 3$ By symmetry considerations together with a measure-theoretic lemma one obtains a subset of the sphere which has area at most $1 / 4$ but whose image under one of the generators has area at least $3 / 4$. A direct computation then shows that this element moves the center a distance at least $\log 3$.

Given a Patterson measure associated to a discrete group $\Gamma$, one can construct a positive, $\Gamma$-invariant (generalized) eigenfunction of the hyperbolic Laplacian by integrating the hyperbolic Poisson kernel against the measure. This eigenfunction has a positive eigenvalue and therefore determines a superharmonic function on $\mathbf{H}^{3} / \Gamma$ (which is a hyperbolic manifold if $\Gamma$ is torsion-free). This allows one to prove that if every superharmonic function on $\mathbf{H}^{3} / \Gamma$ is constant then the area measure is the unique Patterson measure associated to $\Gamma$.

An argument due to Thurston shows that certain geometric conditions on the ends of a complete hyperbolic manifold $M=\mathbf{H}^{3} / \Gamma$ imply that all positive superharmonic functions on $M$ are constant. In our setting the appropriate condition is that there exist a sequence 
of singular surfaces having metrics of intrinsic curvature $\leq-1$ and bounded genera, such that every compact subset of $M$ is "enclosed" in a homological sense by a surface in the sequence. By a theorem of Canary's, such a sequence exists if $\Gamma$ is topologically tame, contains no parabolics and has limit set equal to the entire sphere at infinity of hyperbolic space. Thus we need only to establish the inequality in the conclusion of Theorem 9.1 in the case that the limit set of $\Gamma$ is a proper subset of the sphere at infinity, i.e. when $\Gamma$ has non-empty set of discontinuity.

Notice, however, that if the set of discontinuity is non-empty then the area measure cannot be a Patterson measure. Our strategy is to reduce the case where $\Gamma$ has non-empty set of discontinuity to the more exotic case where the area measure is a Patterson measure by considering limits of groups with non-empty set of discontinuity. There is an open subset $\mathfrak{G} \mathfrak{F}$ of the variety $\mathrm{PSL}_{2}(\mathbf{C}) \times \mathrm{PSL}_{2}(\mathbf{C})$ consisting of all pairs of elements $(\xi, \eta)$ such that $\langle\xi, \eta\rangle$ is a free group of rank 2 having a non-empty set of discontinuity and containing no parabolics. If $(\xi, \eta)$ is any point in the closure $\overline{\mathfrak{G} \mathfrak{F}}$ (in the complex topology) of $\mathfrak{G} \mathfrak{F}$, then $\langle\xi, \eta\rangle$ is still free and discrete. It is easy to show that for any point $z \in \mathbf{H}^{3}$ the function $(\xi, \eta) \mapsto \max (\operatorname{dist}(z, \xi \cdot z), \operatorname{dist}(z, \eta \cdot z))$ is a proper continuous function on $\overline{\mathfrak{G} \mathfrak{F}}$ with no local minimum on $\mathfrak{G} \mathfrak{F}$. Hence it takes a minimum value at some point $(\xi, \eta)$ in the frontier $\mathfrak{B}$ of $\mathfrak{G} \mathfrak{F}$. This reduces the proof of the inequality $\max (\operatorname{dist}(z, \xi \cdot z), \operatorname{dist}(z, \eta \cdot z)) \geq \log 3$ for the case $(\xi, \eta) \in \mathfrak{G} \mathfrak{F}$ to the proof in the case $(\xi, \eta) \in \mathfrak{B}$. We then complete the proof by showing that there is a dense $G_{\delta}$ in $\mathfrak{B}$ consisting of points for which the area measure is indeed a Patterson measure. We use Thurston's criterion here as well; we show that, for $(\xi, \eta)$ in a dense $G_{\delta}$ subset of $\mathfrak{B}$, there exists a sequence of negatively curved singular surfaces of bounded genera enclosing every compact set in $\mathbf{H}^{3} /\langle\xi, \eta\rangle$. The proof of the existence of this dense $G_{\delta}$ contains much of the technical work in the paper.

Each of the singular surfaces that we construct is a map of a closed surface into $M=$ $\mathbf{H}^{3} /\langle\xi, \eta\rangle$. The domain surface is divided into "pairs of pants" by a family of "waist" curves which are mapped to closed geodesics in $M$. Furthermore, the surfaces in the sequence are girded in the sense that the lengths of the waist geodesics tend to 0 through the sequence. The existence of a sequence of singular surfaces of this type is proved by means of a recent result of Curt McMullen's asserting the density of maximal cusps in $\mathfrak{B}$. The singular surfaces are chosen to be invariant with respect to a canonical involution of $M$; such an involution exists because $\pi_{1}(M)$ is a 2-generator group. By combining this invariance property with the fact that the waist lengths tend to 0 , we show that any compact subset of $M$ is enclosed by some surface in the family.

Our construction of girded surfaces works only in the 2-generator case because it uses the involution. However, we believe that a similar picture holds more generally. Let $\Gamma$ be any geometrically finite Kleinian group without parabolics, and let $M$ be the manifold defined by a generic point in the frontier of the set of quasi-conformal deformations of $\Gamma$. We conjecture that $M$ is girded, meaning that there exists a sequence of girded surfaces in $M$ such that every compact subset of $M$ is enclosed by a surface in the sequence. For a precise definition of a girded manifold, see 7.4 .

It is worth noting that the singular surfaces which appear in the proof of Canary's theorem are not required to contain short geodesics. In general a topologically tame manifold with geometrically infinite ends need not contain arbitrarily short geodesics, and 
thus need not be girded. Conversely, it is not clear in general whether a girded manifold with finitely generated fundamental group is topologically tame.

The paper is organized as follows. In the first section we establish notation and collect the basic facts about hyperbolic manifolds which will be needed in the paper. The next two sections discuss the correspondence, via the Poisson kernel, between measures on the sphere at infinity and eigenfunctions of the hyperbolic Laplacian in a $\Gamma$-equivariant setting. In essence this material is classical potential theory, with the Poincaré ball replacing the Euclidean ball.

In Section 4 we present Patterson's ideas in a generalized setting to give a construction of a $\Gamma$-invariant conformal density which respects a given decomposition of a discrete group $\Gamma$. Section 5 contains the argument which is used in proving the main estimate in the case where the area measure is the unique Patterson measure. In Section 6 we describe Thurston's geometric criterion which implies that every positive superharmonic function is constant. We introduce the notion of a Bonahon surface and formulate the criterion in terms of these surfaces.

In Section 7 we develop the notions that we use in constructing our girded surfaces. Section 8 contains the proof of the existence of a dense $G_{\delta}$ in $\mathfrak{B}$ consisting of girded groups. In Section 9 we prove the main theorem and in Section 10 we give the applications to closed manifolds.

The preprint of this paper contains a more self-contained and detailed discussion of the topics in Sections 4 and 6 of the current version. In addition the preprint contains a full development of the geometry of ultra-hyperbolic surfaces. The point of view taken in the preprint is somewhat different from the one taken in [Bo], being based on "packing" arguments similar to some that have been used by Thurston and Gromov.

During the course of this research we have benefited greatly from conversations with Robert Brooks, Richard Canary, Craig Hodgson, Howard Masur, Curt McMullen, Robert Meyerhoff, John Smillie and Jeff Weeks. We are indebted to Mohan Ramachandran for guiding us through the maze of potential theory, and to Francis Bonahon for his patient explanation of his ideas.

\section{$\S 1$ Hyperbolic SPACE AND DisCRETE GROUPS}

We establish some notation and conventions that will be used throughout the paper.

1.1. Hyperbolic space. Whenever we consider a metric space, we shall write dist $(x, y)$ to denote the distance function, provided that no confusion can result. In a metric space we also write $\operatorname{dist}(x, S)$ to denote the distance from a point $x$ to a closed set $S$. If $S$ is a subset of a metric space and $r$ is a positive number, we shall denote the closed $r$-neighborhood of $S$ by $\operatorname{nbhd}_{r}(S)$.

By a model of hyperbolic $n$-space, for $n \geq 2$, we shall mean a complete, 1-connected Riemannian $n$-manifold of constant sectional curvature -1 . Throughout this paper we shall fix, abstractly, a model $\mathbf{H}^{n}$ of hyperbolic space. Any two models of hyperbolic $n$-space are isometric, and the isometry group of any model acts transitively on its orthonormal frame bundle. It will frequently be useful to identify $\mathbf{H}^{n}$, via a suitable isometry, with one 
of the standard concrete models. The concrete models which will be used most often are the upper half-space model, which has underlying space $\mathbf{R}^{n-1} \times[0, \infty)$, and the Poincaré model, for which has underlying space is the open unit ball $B^{n} \in \mathbf{R}^{n}$. The geodesics in these models are intersections with lines or circles in the ambient euclidean space which are orthogonal, respectively, to the plane $x_{n+1}=0$ or the unit sphere. In studying convex sets in $\mathbf{H}^{n}$ it is useful to consider the projective model, which also has underlying space $B^{n}$, but with a metric for which the geodesics are intersections of euclidean geodesics with $B^{n}$. For detailed descriptions of these spaces, and for general information on hyperbolic geometry, the reader is referred to $[\mathrm{F}]$.

The sphere at infinity of $\mathbf{H}^{n}$ will be denoted by $S_{\infty}^{n-1}$ or simply $S_{\infty}$. The canonical compactification of $\mathbf{H}^{n}$, of which $S_{\infty}$ is the boundary, will be denoted $\overline{\mathbf{H}}^{n}$. Any selfisometry $\gamma$ of $\mathbf{H}^{n}$ extends to a conformal automorphism of $\overline{\mathbf{H}}^{n}$, which we denote $\bar{\gamma}$. We denote by $\gamma_{\infty}$ the conformal automorphism of $S_{\infty}$ obtained by restricting $\bar{\gamma}$.

Given any point $z \in \mathbf{H}^{n}$, there exists an isometry $h$ from $\mathbf{H}^{n}$ onto the Poincaré model $B^{n}$ which maps $z$ to 0 . Furthermore, $h$ is unique modulo composition with orthogonal linear transformations of $\mathbf{R}^{n}$. It follows that if we pull back the metric of $S^{n-1}=\partial B^{n}$ via $\bar{h}$, we obtain a metric on $S_{\infty}$ which is uniquely determined by the point $z$; we call it the round metric centered at $z$.

Any isometry $h$ of the upper half-space model onto $\mathbf{H}^{n}$ extends uniquely to a conformal diffeomorphism of $\mathbf{R}^{n-1} \times[0, \infty)$ onto $\overline{\mathbf{H}}^{n}-\{\zeta\}$, where $\zeta \in S_{\infty}$ is a point determined by $h$. We shall say that $h$ maps $\infty$ to $\zeta$ (or that $h^{-1}$ maps $\zeta$ to $\infty$ ). Given any point $\zeta \in S_{\infty}$, there exists an isometry of $\mathbf{R}^{n-1} \times(0, \infty)$ onto $\mathbf{H}^{n}$ which maps $\infty$ to $\zeta$.

If $A_{1}$ and $A_{2}$ are points of $\overline{\mathbf{H}}^{n}$ and $B$ is a point of $\mathbf{H}^{n}$, we denote by $\angle A_{1} B A_{2}$ the angle between the ray from $B$ to $A_{1}$ and the ray from $B$ to $A_{2}$; here by the ray from $B$ to $A_{i}$ we mean the (hyperbolic) ray starting at $B$ and passing through $A_{i}$ (if $A_{i} \in \mathbf{H}^{n}$ ) or having $A_{i}$ as an endpoint (if $A_{i} \in S_{\infty}$ ).

1.2. Convexity. A subset $K$ of $\overline{\mathbf{H}}^{n}$ is termed convex if $K \neq \emptyset$ and if every line segment in $\mathbf{H}^{n}$ with endpoints in $K$ is itself contained in $K$; here a "line segment" may be an entire line or a half-infinite ray. The convex hull of a non-empty subset $X$ of $\overline{\mathbf{H}}^{n}$ is defined to be the intersection of all convex subsets of $\overline{\mathbf{H}}^{n}$ containing $X$, and will be denoted $\overline{\operatorname{hull}}(X)$. We set $\operatorname{hull}(X)=\overline{\operatorname{hull}}(X) \cap \mathbf{H}^{n}$. Clearly $\overline{\operatorname{hull}}(X)$ and hull $(X)$ are convex.

Any isometry of $\mathbf{H}^{n}$ onto the projective model $B^{n}$ extends to a diffeomorphism of $\overline{\mathbf{H}}^{n}$ onto $\bar{B}^{n}$ which maps convex subsets of $\overline{\mathbf{H}}^{n}$ onto convex subsets (in the usual Euclidean sense) of $\bar{B}^{n}$. Hence any compact convex subset of $\overline{\mathbf{H}}^{n}$ which has non-empty interior is homeomorphic to a closed $n$-ball. It follows that any closed convex subset of $\mathbf{H}^{n}$ is the intersection of $\mathbf{H}^{n}$ with a topological closed $n$-ball in $\overline{\mathbf{H}}^{n}$, and is therefore a contractible $n$-manifold-with-boundary.

Proposition. If $K$ is a convex subset of $\mathbf{H}^{n}$, then for any positive number $R$ the set $\operatorname{nbhd}_{R}(K)$ is convex.

Proof. It suffices to show that if $\alpha \subset \mathbf{H}^{n}$ is a compact line segment then $\operatorname{nbhd}_{R}(\alpha)$ is convex. This is in turn equivalent to showing that for any line $\ell \subset \mathbf{H}^{n}$ the set $\ell \cap n b h d_{R}(\alpha)$ is connected. If $\varphi: \mathbf{H}^{n} \rightarrow \mathbf{R}$ is the continuous function that assigns to each point of $\mathbf{R}^{n}$ its minimum distance from $\alpha$, we have $\ell \cap \operatorname{nbhd}_{R}(\alpha)=(\varphi \mid \ell)^{-1}([0, R))$. It therefore suffices 
to prove that the function $\varphi \mid \ell$ has no local maximum. This is an exercise in hyperbolic geometry which we leave to the reader.

1.3. Let $\gamma$ be any non-trivial orientation-preserving isometry of $\mathbf{H}^{3}$. For any $\epsilon>0$ let us write

$$
C_{\epsilon}(\gamma)=\left\{z \in \mathbf{H}^{3}: \operatorname{dist}(z, \gamma \cdot z) \leq \epsilon\right\}
$$

If $\gamma$ is parabolic, i.e. if $\gamma_{\infty}$ has a unique fixed point $\zeta \in S_{\infty}$, then $C_{\epsilon}(\gamma)$ is a closed horoball based at $\zeta$.

If $\gamma$ is loxodromic then $\gamma_{\infty}$ has exactly two fixed points, say $\zeta_{-}$and $\zeta_{+}$, in $S_{\infty}$. The line $A$ joining $\zeta_{-}$and $\zeta_{+}$is by definition the axis of $\gamma$, and $\gamma \mid A$ is a translation through some distance length $(\gamma)$. The distance from a point to its image under $\gamma$ depends on the twist angle of $\gamma$ as well as on length $(\gamma)$ and the distance of the point from the axis $A$. However, the following result is sufficient to permit us to describe $C_{\epsilon}(\gamma)$ in this case.

Proposition. Let $\gamma$ be a loxodromic isometry of $\mathbf{H}^{3}$ with axis $A$. Then there is a monotonically increasing continuous function $f=f_{\gamma}:[0, \infty) \rightarrow[0, \infty)$ such that $\operatorname{dist}(z, \gamma \cdot z)=$ $f(\operatorname{dist}(z, A))$ for every point $z \in \mathbf{H}^{n}$.

Proof. Left to the reader.

It follows from the above proposition that if $\gamma$ is loxodromic with axis $A$ then we have $C_{\epsilon}(\gamma)=\emptyset$ for any $\epsilon<\operatorname{length}(\gamma)$, and for any $\epsilon \geq \operatorname{length}(\gamma)$ we have $C_{\epsilon}(\gamma)=\operatorname{nbhd}_{\delta}\left(A_{\gamma}\right)$, where $\delta$ is a non-negative number depending on $\gamma$ and $\epsilon$. (In the notation of the proposition we have $\delta=f_{\gamma}(\epsilon)$.)

1.4. By a Kleinian group we shall mean a discrete group of orientation-preserving isometries of $\mathbf{H}^{3}$ which is non-elementary in the sense that it has no abelian subgroup of finite index. If $\Gamma$ is a Kleinian group we shall write $\Lambda_{\Gamma}$ for its limit set.

When $\Gamma$ is a torsion-free Kleinian group we shall write $M(\Gamma)$ for the complete hyperbolic 3-manifold $\mathbf{H}^{3} / \Gamma$. The set hull $\left(\Lambda_{\Gamma}\right)$ is closed since $\Lambda_{\Gamma}$ is compact, and non-empty since $\Gamma$ is non-elementary. Thus the set $\operatorname{nbhd}_{1}\left(\operatorname{hull}\left(\Lambda_{\Gamma}\right)\right) \subset \mathbf{H}^{3}$ is closed and has non-empty interior. It is convex by Proposition 1.2 , and is clearly $\Gamma$-invariant. Hence by $1.2, \operatorname{nbhd}_{1}\left(\operatorname{hull}\left(\Lambda_{\Gamma}\right)\right)$ is a contractible, $\Gamma$-invariant 3 -manifold-with-boundary. We shall write

$$
N(\Gamma)=\left(\operatorname{nbhd}_{1}\left(\operatorname{hull}\left(\Lambda_{\Gamma}\right)\right)\right) / \Gamma \subset M(\Gamma)
$$

By the discussion above, $N(\Gamma)$ is a 3-manifold-with-boundary and a deformation retract of $M(\Gamma)$.

1.5. Margulis numbers. The group of all orientation-preserving isometries of $\mathbf{H}^{3}$ is isomorphic to $\mathrm{PSL}_{2}(\mathbf{C})$. In $\mathrm{PSL}_{2}(\mathbf{C})$, every element of order $>2$ has an abelian centralizer. Hence if $\Gamma$ is a torsion-free Kleinian group, every non-trivial element of $\Gamma$ lies in a unique maximal abelian subgroup. If $H$ is any maximal abelian subgroup of $\Gamma$ then either $H$ is cyclic and $H-\{1\}$ consists of loxodromic elements with a common axis, or $H$ is free abelian of rank 1 or 2 and $H-\{1\}$ consists of parabolic elements with a common fixed point in $S_{\infty}$. In the latter case $H$ will be called a cuspidal subgroup of $\Gamma$. 
For any point $P \in M(\Gamma)$ we shall denote by $\operatorname{short}(P)$ the infimum of the lengths of all homotopically non-trivial loops based at $P$. Thus for any $z \in \mathbf{H}^{3}$ lying in the fiber above $P$ we have

$$
\operatorname{short}(P)=\inf _{1 \neq \gamma \in \Gamma} \operatorname{dist}(z, \gamma \cdot z)
$$

We have $0<\operatorname{short}(P) \leq \infty$, and $\operatorname{short}(P)=\infty$ if and only if $\Gamma=\{1\}$. When $\Gamma \neq\{1\}$, the infimum in the definition of $\operatorname{short}(P)$ is realized. For any interval $I \subset(0, \infty)$ we denote by $M_{I}(\Gamma)$ the set of all points $P \in M(\Gamma)$ such that $\operatorname{short}(P) \in I$. Thus for any $\epsilon>0$, the set $M_{(0, \epsilon]}$ consists of all points of $M$ through which there pass homotopically non-trivial closed curves of length $\leq \epsilon$. We call $M_{(0, \epsilon]}$ the $\epsilon$-thin part of $M$.

Let $\widetilde{M}_{(0, \epsilon]}$ denote the pre-image of $M_{(0, \epsilon]}$ in $\mathbf{H}^{n}$. Then by definition, $\widetilde{M}_{(0, \epsilon]}$ consists of all points $z \in \mathbf{H}^{3}$ such that $\operatorname{dist}(z, \gamma \cdot z) \leq \epsilon$ for some $\gamma \in \Gamma-\{1\}$. Hence we have

$$
\widetilde{M}_{(0, \epsilon]}=\bigcup_{1 \neq \gamma \in \Gamma} C_{\epsilon}(\gamma) .
$$

For any maximal abelian subgroup $H$ of $\Gamma$ and for any fixed $\epsilon>0$, the family of sets $\left\{C_{\epsilon}(\gamma): \gamma \in \Gamma\right\}$ is totally ordered by inclusion. It follows from the discreteness of $\Gamma$ that there is always a maximal set $C_{\epsilon}(H)$ in this family. Thus for any $\epsilon>0$ we may write

$$
\widetilde{M}_{(0, \epsilon]}=\bigcup_{H} C_{\epsilon}(H)
$$

where $H$ ranges over all maximal abelian subgroups of $\Gamma$. The discreteness of $\Gamma$ also implies that the sets $C_{\epsilon}(H)$ form a locally finite family. A positive number $\epsilon$ will be called a Margulis number for $M(\Gamma)$ if the sets $C_{\epsilon}(H)$, where $\epsilon$ ranges over the distinct maximal abelian subgroups of $\Gamma$, are pairwise disjoint. Equivalently, $\epsilon$ is a Margulis number for $M$ if for every point $z \in \mathbf{H}^{3}$ and every pair of non-commuting elements $\xi, \eta$ of $\Gamma$, we have $\max (\operatorname{dist}(z, \xi \cdot z), \operatorname{dist}(z, \eta \cdot z)) \geq \epsilon$. There exists a positive number which is a 3 dimensional Margulis constant in the sense that it is a Margulis number for every complete hyperbolic 3-manifold. The existence of such a constant follows, for example, from [Be, Theorem 5.4.5].

If $\epsilon$ is a Margulis number for $M$ then each component of $M_{(0, \epsilon]}$ has the form $C_{\epsilon}(H) / H$ for some maximal abelian subgroup $H$ of $\Gamma$. When $H$ is cuspidal, so that $C_{\epsilon}(H)$ is a horoball, we call $C_{\epsilon}(H) / H$ a standard cusp neighborhood. When $H$ consists of loxodromic elements, it is cyclic. In this case it follows from Proposition 1.3 that $C_{\epsilon}(H)=\emptyset$ when $\epsilon$ is less than the length of a generator of $H$, and that otherwise $C_{\epsilon}(H)=\operatorname{nbhd}_{\delta}(A)$, where $A$ is the axis of a generator of $H$ and $\delta$ is a non-negative number depending on $\epsilon$ and $H$. In particular, $A / H$ is a simple closed geodesic of length $\leq \epsilon$ and $\mathcal{T}=\operatorname{nbhd}_{\delta}(A / H)$. Topologically, $\mathcal{T}$ is a solid torus unless $A / H$ has length $\epsilon$, in which case $\mathcal{T}=A / H$. When $H$ consists of loxodromic elements and $C_{\epsilon}(H) \neq \emptyset$, we call $\mathcal{T}=C_{\epsilon}(H) / H$ a tube.

1.6. If $\Gamma$ is any Kleinian group and $\epsilon$ is any Margulis number for $M(\Gamma)$, we shall denote by $M_{(0, \epsilon]}^{c}(\Gamma)$ the union of all components of $M_{(0, \epsilon]}(\Gamma)$ which are standard cusp neighborhoods. We shall write

$$
M_{\{\epsilon\}}^{c}(\Gamma)=\partial M_{(0, \epsilon]}^{c}(\Gamma) \subset M_{\{\epsilon\}}(\Gamma)
$$


and

$$
M_{[\epsilon, \infty)}^{c}(\Gamma)=M(\Gamma)-\operatorname{int} M_{(0, \epsilon]}^{c}(\Gamma) \supset M_{[\epsilon, \infty)}(\Gamma) .
$$

Furthermore, if $I$ is any of the intervals $(0, \epsilon],\{\epsilon\}$ or $[\epsilon, \infty)$, we set

$$
N_{I}^{c}(\Gamma)=N(\Gamma) \cap M_{I}^{c}(\Gamma) .
$$

The pre-image of $N_{I}^{c}(\Gamma)$ in $\mathbf{H}^{3}$ will be denoted $\tilde{N}_{I}^{c}(\Gamma)$.

For any Margulis number $\epsilon$, the set $N_{(0, \epsilon]}(\Gamma)$ is a 3-manifold-with-boundary and a deformation retract of $M_{(0, \epsilon]}(\Gamma)$. Indeed, each component of $M_{(0, \epsilon]}^{c}(\Gamma)$ has the form $C_{\epsilon}(H) / H$ for some maximal abelian subgroup $H$ of $\Gamma$ consisting of parabolic elements. The fixed point $\zeta \in S_{\infty}$ belongs to $\Lambda_{\Gamma}$, and we have $\Lambda_{\Gamma} \neq\{\zeta\}$ since $\Gamma$ is non-elementary; hence $\operatorname{hull}\left(\Lambda_{\Gamma}\right) \cap C_{\epsilon}(H) \neq \emptyset$. Since $\operatorname{hull}\left(\Lambda_{\Gamma}\right)$ and $C_{\epsilon}(H)$ are compact and convex, it follows that $\operatorname{nbhd}_{1}\left(\operatorname{hull}\left(\Lambda_{\Gamma}\right)\right) \cap C_{\epsilon}(H)$ is a closed convex subset of $\mathbf{H}^{3}$ with non-empty interior, and by 1.2 is therefore a contractible 3-manifold-with-boundary. Hence the quotient of $\operatorname{nbhd}_{1}\left(\operatorname{hull}\left(\Lambda_{\Gamma}\right)\right) \cap C_{\epsilon}(H)$ by the action of $H$ is a 3-manifold-with-boundary and a deformation retract of $C_{\epsilon}(H) / H$; our assertion follows.

1.7. Geometric finiteness. The Kleinian group $\Gamma$ is said to be geometrically finite if $N_{[\epsilon, \infty)}^{c}(\Gamma)$ is compact for some Margulis number $\epsilon$.

If $\Gamma$ is geometrically finite then it has only finitely many conjugacy classes of cuspidal subgroups. Indeed, if $\epsilon$ is a Margulis constant, the sets $C_{\epsilon}(H)$, where $H$ ranges over the cuspidal subgroups of $\Gamma$, form a locally finite family (1.5). Hence the corresponding cusp neighborhoods $C_{\epsilon}(H) / H$ form a locally finite family of subsets of $M(\Gamma)$. For each $H$ the fixed point $\zeta$ of $H$ belongs to $\Lambda_{\Gamma}$, and $\Lambda_{\Gamma} \neq\{\zeta\}$ since $\Gamma$ is non-elementary; hence hull $\left(\Lambda_{\Gamma}\right)$ contains a geodesic with one endpoint at $\zeta$. It follows that $C_{\epsilon}(H) / H \cap N_{[\epsilon, \infty)}^{c}(\Gamma) \neq \emptyset$ for every $H$. Hence if $N_{[\epsilon, \infty)}^{c}(\Gamma)$ is compact then the family of cusp neighborhoods $C_{\epsilon}(H) / H$ is finite, establishing our assertion.

Lemma. Let $H$ be a discrete group of parabolic isometries of the upper half-space model of hyperbolic space, with common fixed point $\infty$. Let $\Lambda$ be a closed non-empty $H$-invariant subset of $\mathbf{R}^{2} \times\{0\}$ such that $\Lambda / H$ is compact.

(i) If $H$ is free abelian of rank 2 then $\mathbf{R}^{2} \times\{h\} \subset \operatorname{hull}(\Lambda \cup\{\infty\})$ for all sufficiently large $h$.

(ii) If $H$ is infinite cyclic then there is an $H$-invariant strip $S$ bounded by two (possibly equal) parallel lines in $\mathbf{R}^{2}$ such that $\Lambda \subset S$ and $\mathbf{R}^{2} \times\{h\} \cap \operatorname{hull}(\Lambda \cup\{\infty\})=S \times\{h\}$ for all sufficiently large $h$.

Proof. By convexity, if a point $P$ of upper-half space is contained in $\operatorname{hull}(\Lambda \cup\{\infty\})$ then so is the entire vertical ray beginning at $P$. Now suppose that $\alpha$ and $\beta$ are points of $\Lambda$ and that the Euclidean distance from $\alpha$ to $\beta$ is $d$. Let $\sigma$ be the Euclidean line segment from $\alpha$ to $\beta$. Since the hyperbolic geodesic from $\alpha$ to $\beta$ is a Euclidean circle of radius $d / 2$, it follows that for all $h>d / 2$ we have $\sigma \times\{h\} \subset \operatorname{hull}(\Lambda \cup\{\infty\})$.

Let $\gamma \in H$ and $\alpha \in \Lambda$ and let $\ell$ be the line in $\mathbf{R}^{2}$ which contains the points $\bar{\gamma}^{n}(\alpha)$ for $n \in \mathbf{Z}$. Let $d$ be the Euclidean distance from $\bar{\gamma}^{n}(\alpha)$ to $\bar{\gamma}^{n+1}(\alpha)$. By the observation above, applied to each of the pairs $\left\{\bar{\gamma}^{n}(\alpha), \bar{\gamma}^{n+1}(\alpha)\right\}$ we have $\ell \times\{h\} \subset \operatorname{hull}(\Lambda \cup\{\infty\})$ for all $h>d / 2$. 
Suppose that $H$ is free abelian of rank 2 with generators $\gamma_{1}$ and $\gamma_{2}$. Let $d_{1}$ and $d_{2}$ be the respective translation lengths of $\bar{\gamma}_{1}$ and $\bar{\gamma}_{2}$ acting on $\mathbf{R}^{2} \times\{0\}$. Let $\alpha$ be a point of $\Lambda$. Let $\ell_{n}$ denote the $\bar{\gamma}_{1}$-invariant line through $\bar{\gamma}_{2}^{n}(\alpha)$. For all $h>d_{1} / 2$ we have that $\ell_{n} \times\{h\} \subset \mathbf{R}^{2} \times\{h\} \cap \operatorname{hull}(\Lambda \cup\{\infty\})$. Every point of $\mathbf{R}^{2}$ lies on a line segment of length $d_{2}$ with endpoints in $\ell_{n}$ and $\ell_{n+1}$ for some $n \in \mathbf{Z}$. It follows that for $h>d_{1} / 2+d_{2} / 2$ we have $\mathbf{R}^{2} \times\{h\} \subset \operatorname{hull}(\Lambda \cup\{\infty\})$.

Suppose that $H$ is infinite cyclic with generator $\gamma$ and that $\bar{\gamma}$ acts on $\mathbf{R}^{2} \times\{0\}$ as a Euclidean translation through a distance $d$. The assumption that $\Lambda / H$ is compact implies that $\Lambda$ is contained in a strip $S$ bounded by $\bar{\gamma}$-invariant lines $\ell_{1}$ and $\ell_{2}$. Since $\Lambda$ is closed, the lines $\ell_{1}$ and $\ell_{2}$ may be taken to contain points of $\Lambda$. If the Euclidean distance from $\ell_{1}$ to $\ell_{2}$ is $w$ then for all $h>d / 2+w / 2$ we have $S \times\{h\} \subset \operatorname{hull}(\Lambda \cup\{\infty\})$. On the other hand, $S \times[0, \infty)$ is a convex set containing $\Lambda \cup\{\infty\}$. Therefore $\mathbf{R}^{2} \times\{h\} \cap \operatorname{hull}(\Lambda \cup\{\infty\})=S \times\{h\}$.

Recall that a topological 3-manifold $N$ is said to be irreducible if every locally flat 2 -sphere in $N$ is the boundary of a 3 -ball in $N$.

Proposition. Let $\Gamma$ be a geometrically finite, torsion-free Kleinian group; set $M=M(\Gamma)$ and $N=N(\Gamma)$. Then $N_{[\epsilon, \infty)}^{c}$ is compact whenever $\epsilon$ is a Margulis number for $M$. Furthermore, for every sufficiently small Margulis number $\epsilon_{0}$ the following conclusions hold:

(i) $N_{\left[\epsilon_{0}, \infty\right)}^{c}$ is an orientable, irreducible 3-manifold-with-boundary and is a deformation retract of $N$ and hence of $M$; and

(ii) $N_{\left\{\epsilon_{0}\right\}}^{c}$ is a compact 2-manifold-with-boundary, properly embedded in $N$, and is a deformation retract of $N_{\left(0, \epsilon_{0}\right]}$ and hence of $M_{\left(0, \epsilon_{0}\right]}$.

Proof. Since $\Gamma$ is geometrically finite, there is a Margulis number $\epsilon_{1}$ for $M$ such that $N_{\left[\epsilon_{1}, \infty\right)}^{c}$ is compact.

Let $\epsilon$ be any Margulis number. In order to show that $N_{[\epsilon, \infty)}^{c}$ is compact, we observe that for any sufficiently small $\epsilon^{\prime}>0$ we have

$$
\widetilde{N}_{[\epsilon, \infty)}^{c} \subset \operatorname{nbhd}_{1}\left(\operatorname{hull}\left(\Lambda_{\Gamma}\right) \cap \widetilde{M}_{\left[\epsilon^{\prime}, \infty\right)}^{c}\right) .
$$

Hence we need only show that $\left(\operatorname{hull}\left(\Lambda_{\Gamma}\right) \cap \widetilde{M}_{\left[\epsilon^{\prime}, \infty\right)}^{c}\right) / \Gamma$ is compact for sufficiently small $\epsilon^{\prime}$. In particular we may suppose that $\epsilon^{\prime}<\epsilon_{1}$. Since $\left(\operatorname{hull}\left(\Lambda_{\Gamma}\right) \cap \widetilde{M}_{\left[\epsilon_{1}, \infty\right)}^{c}\right) / \Gamma$ is compact, and since $\Gamma$ has only finitely many conjugacy classes of cuspidal subgroups by 1.7 , we need only show that for any cuspidal subgroup $H$ of $\Gamma$ the set $\left(\operatorname{hull}\left(\Lambda_{\Gamma}\right) \cap\left(C_{\epsilon_{1}}(H)-C_{\epsilon}(H)\right)\right) / H$ has compact closure in $M$. For this we identify $\mathbf{H}^{3}$ with the upper half-space model by an isometry mapping the fixed point of $H$ to $\infty$ and set $\Lambda=\Lambda_{\Gamma}-\{\infty\}$. Let $h$ and $h_{1}$ be real numbers so that the boundaries of the horoballs $C_{\epsilon}(H)$ and $C_{\epsilon_{1}}(H)$ are identified with the horizontal planes $\mathbf{R}^{2} \times\{h\}$ and $\mathbf{R}^{2} \times\left\{h_{1}\right\}$ respectively. If $H$ has rank 2 then

$$
\left(C_{\epsilon_{1}}(H)-C_{\epsilon}(H)\right) / H \subset\left(\mathbf{R}^{2} \times\left[h_{1}, h\right]\right) / H,
$$

the set on the right being compact. If $H$ has rank 1 then

$$
\left(\operatorname{hull}\left(\Lambda_{\Gamma}\right) \cap\left(C_{\epsilon_{1}}(H)-C_{\epsilon}(H)\right)\right) / H \subset\left(S \times\left[h_{1}, h\right]\right) / H,
$$


where $S$ is the strip provided by the lemma. Again, the set on the right is compact.

Next we prove that for a sufficiently small Margulis number $\epsilon_{0}$ the set $N_{\left\{\epsilon_{0}\right\}}^{c}$ is a compact 2-manifold-with-boundary properly embedded in $N$ (which by 1.4 is a 3-manifold-withboundary), and that $N_{\left\{\epsilon_{0}\right\}}^{c}$ is a deformation retract of $N_{\left(0, \epsilon_{0}\right]}$. This will establish assertion (ii) of the proposition, since $N_{\left(0, \epsilon_{0}\right]}$ is a deformation retract of $M_{\left(0, \epsilon_{0}\right]}$ according to 1.6. Since $\Gamma$ has only finitely many conjugacy classes of cuspidal subgroups, it suffices to show that for any cuspidal subgroup $H$ of $\Gamma$ there is a Margulis number $\epsilon_{H}$ such that for any $\epsilon \leq \epsilon_{H}$ the set $N \cap\left(\partial C_{\epsilon}(H) / H\right)$ is a properly embedded 2-manifold-with-boundary in $N$ and is a deformation retract of $C_{\epsilon}(H) / H$. Equivalently, we must show that for small enough $\epsilon$ the set $\operatorname{nbhd}_{1}\left(\operatorname{hull}\left(\Lambda_{\Gamma}\right)\right) \cap \partial C_{\epsilon}(H)$ is a contractible, properly embedded 2-manifold-withboundary in $\operatorname{nbhd}_{1}\left(\operatorname{hull}\left(\Lambda_{\Gamma}\right)\right)$.

We prove this by applying the lemma as before. The statement follows immediately in the case where $H$ is free abelian of rank 2. In the case that $H$ is isomorphic to $\mathbf{Z}$ let $C$ denote the horoball $\mathbf{R}^{2} \times\left[e^{c}, \infty\right)$, and $D$ denote the region $S \times(0, \infty)$ where $S$ is the strip provided by the conclusion of the lemma. We have $\operatorname{hull}\left(\Lambda_{\Gamma}\right) \cap C=D \cap C$. Hence if $C^{\prime}$ denotes the horoball $\mathbf{R}^{2} \times\left[e^{c+1}, \infty\right) \subset C$, so that the minimum distance between

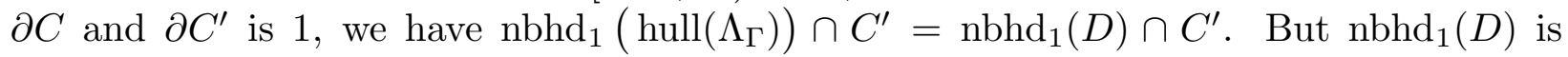
bounded by two equidistant surfaces [F, p. 39] with the two boundary components of $D$ as axial planes; these equidistant surfaces are intersections of the upper half-space with non-horizontal Euclidean planes $E_{1}$ and $E_{2}$ in $\mathbf{R}^{3}$.

We have $C^{\prime}=C_{\epsilon}(H)$ for some Margulis number $\epsilon_{H}$; and for any $\epsilon \leq \epsilon_{H}$, the set $\operatorname{nbhd}_{1}\left(\operatorname{hull}\left(\Lambda_{\Gamma}\right)\right) \cap \partial C_{\epsilon}(H)$ is the closed strip in the horizontal Euclidean plane $\partial C_{\epsilon}(H)$ bounded by the lines $\partial C_{\epsilon}(H) \cap E_{1}$ and $\partial C_{\epsilon}(H) \cap E_{2}$. Hence $\operatorname{nbhd}_{1}\left(\operatorname{hull}\left(\Lambda_{\Gamma}\right)\right) \cap \partial C_{\epsilon}(H)$ is a contractible 2-manifold-with-boundary. By inspection we have that $\operatorname{nbhd}_{1}\left(\operatorname{hull}\left(\Lambda_{\Gamma}\right)\right) \cap$ $\partial C_{\epsilon}(H)$ is properly embedded in $\operatorname{nbhd}_{1}\left(\operatorname{hull}\left(\Lambda_{\Gamma}\right)\right)$. This proves (ii) for small $\epsilon_{0}$.

We shall complete the proof by showing that (ii) implies (i). If (ii) holds for a given $\epsilon_{0}$ then in particular $N_{\left\{\epsilon_{0}\right\}}^{c}$ is a deformation retract of $N_{\left(0, \epsilon_{0}\right]}^{c}$; thus $N_{\left[\epsilon_{0}, \infty\right)}^{c}$ is a deformation retract of $N$, and hence (by 1.4) of $M$. In particular $N_{\left[\epsilon_{0}, \infty\right)}^{c}$ is aspherical. Since $M$ is covered by $\mathbf{H}^{3}$, every locally flat 2 -sphere $S$ in $M(\Gamma)$ bounds a unique ball $B \subset M$; if $S \subset N_{\left[\epsilon_{0}, \infty\right)}^{c}$ then $B \subset N_{\left[\epsilon_{0}, \infty\right)}^{c}$ by asphericity. Hence $N_{\left[\epsilon_{0}, \infty\right)}^{c}$ is irreducible. Since $M$ is orientable, so is $N_{\left[\epsilon_{0}, \infty\right]}^{c}$. This establishes (i).

1.8. The following proposition about 2-generator groups of isometries of $\mathbf{H}^{3}$ follows easily from results which can be found in $[\mathrm{J} \varnothing \mathrm{r}]$ and in $[\mathrm{F}, \mathrm{V} .1]$.

If $\ell$ is any (geodesic) line in $\mathbf{H}^{3}$, we denote by $\tau_{\ell}$ the $180^{\circ}$ rotation about $\ell$. Thus $\tau_{\ell}$ is the unique element of order 2 in the group Isom $\left(\mathbf{H}^{3}\right)$ whose fixed point set is $\ell$.

Proposition. Let $\xi$ and $\eta$ be two orientation-preserving isometries of $\mathbf{H}^{3}$. Suppose that $\xi_{\infty}$ and $\eta_{\infty}$ have no common fixed point in $S_{\infty}$. Then there is a unique line $\ell \subset \mathbf{H}^{3}$ such that $\tau=\tau_{\ell}$ satisfies $\tau \xi \tau=\xi^{-1}$ and $\tau \eta \tau=\eta^{-1}$.

If $\xi$ and $\eta$ satisfy the hypotheses of the above proposition, the line $\ell$ given by the proposition will be denoted $\ell(\xi, \eta)$. This line is the common perpendicular to the axes of $\xi$ and $\eta$, suitably interpreted in the degenerate cases where one or both of these isometries are parabolic [Fe, III.3 and V.1]. 
1.9. The line $\ell(\xi, \eta)$ is easily seen to depend continuously on $\xi$ and $\eta$ in the following sense. Suppose that $\xi_{\infty}$ and $\eta_{\infty}$ satisfy the hypotheses of Proposition 1.11, and let $\left(\xi_{i}\right)$ and $\left(\eta_{i}\right)$ be sequences of isometries of $\mathbf{H}^{3}$ converging to $\xi_{\infty}$ and $\eta_{\infty}$ respectively, so that $\xi_{i}$ and $\eta_{i}$ also satisfy the hypotheses of the proposition for large $i$. Let $\zeta_{\infty}^{+}$and $\zeta_{\infty}^{-}$denote the endpoints of $\ell\left(\xi_{\infty}, \eta_{\infty}\right)$. Then we may label the endpoints of $\ell\left(\xi_{i}, \eta_{i}\right)$ (for large $i$ ) as $\zeta_{i}^{+}$and $\zeta_{i}^{-}$in such a way that $\zeta_{i}^{+} \rightarrow \zeta_{\infty}^{+}$and $\zeta_{i}^{-} \rightarrow \zeta_{\infty}^{-}$as $i \rightarrow \infty$.

\section{$\S 2$. The PoIsson KeRneL}

We shall need some elementary facts about the Poisson kernel for hyperbolic space. In this section and the next we present the relevant material from a geometric point of view which is natural for the applications in this paper.

2.1. We define a continuous function $\Pi: \mathbf{H}^{n} \times \mathbf{H}^{n} \times \mathbf{H}^{n} \rightarrow \mathbf{R}$ by

$$
\Pi\left(z, z^{\prime}, w\right)=\exp \left(\operatorname{dist}(z, w)-\operatorname{dist}\left(z^{\prime}, w\right)\right) .
$$

From the hyperbolic law of cosines [F, p. 91] we have

$$
\frac{\cosh \operatorname{dist}(z, w)}{\cosh \operatorname{dist}\left(z^{\prime}, w\right)}=\left(\cosh \operatorname{dist}\left(z, z^{\prime}\right)-\tanh \operatorname{dist}(z, w) \sinh \operatorname{dist}\left(z, z^{\prime}\right) \cos \angle z^{\prime} z w\right)^{-1} .
$$

It follows that the function $\Pi$ has a unique positive continuous extension $\bar{\Pi}: \mathbf{H}^{n} \times \mathbf{H}^{n} \times$ $\overline{\mathbf{H}}^{n} \rightarrow \mathbf{R}$. The restriction of $\bar{\Pi}$ to a function on $\mathbf{H}^{n} \times \mathbf{H}^{n} \times S_{\infty}$ will be denoted $\mathcal{P}$. This restriction is given explicitly by

$$
\mathcal{P}\left(z, z^{\prime}, \zeta\right)=\left(\cosh \operatorname{dist}\left(z, z^{\prime}\right)-\sinh \operatorname{dist}\left(z, z^{\prime}\right) \cos \angle z^{\prime} z \zeta\right)^{-1}
$$

for any $z \neq z^{\prime} \in \mathbf{H}^{n}$ and any $\zeta \in S_{\infty}^{n-1}$; and by $\mathcal{P}(z, z, \zeta)=1$ for any $z \in \mathbf{H}^{n}$ and any $\zeta \in S_{\infty}^{n-1}$.

2.2. It is immediate from the definition of the function $\Pi$ that for any points $z, z^{\prime}, z^{\prime \prime}, w \in$ $\mathbf{H}^{n}$ we have $\Pi\left(z, z^{\prime \prime}, w\right)=\Pi\left(z, z^{\prime}, w\right) \Pi\left(z^{\prime}, z^{\prime \prime}, w\right)$. Hence in view of the continuity of $\bar{\Pi}$ we have

$$
\mathcal{P}\left(z, z^{\prime \prime}, \zeta\right)=\mathcal{P}\left(z, z^{\prime}, \zeta\right) \mathcal{P}\left(z^{\prime}, z^{\prime \prime}, \zeta\right)
$$

for any $z, z^{\prime}, z^{\prime \prime} \in \mathbf{H}^{n}$ and any $\zeta \in S_{\infty}$.

2.3. There is a simple formula for the function $\mathcal{P}$ in terms of the upper half-space model. Given any point $\zeta \in S_{\infty}^{n-1}$, let us fix an isometry $J$ of $\mathbf{H}^{n}$ onto the upper half-space model $\mathbf{R}^{n-1} \times(0, \infty)$ which maps $\zeta$ to $\infty$ (see 1.1). The $n$-th coordinate function on $\mathbf{R}^{n-1} \times(0, \infty)$ pulls back via $J$ to a function $\operatorname{Im}: \mathbf{H}^{n} \rightarrow(0, \infty)$. The effect of replacing $J$ by another isometry mapping $\zeta$ to $\infty$ would be to multiply the function Im by a constant; this is because a self-isometry of the upper half-space model that fixes $\infty$ is of the form $x \mapsto \alpha \sigma(x)$, where $\alpha$ is a positive constant and $\sigma$ is a Euclidean isometry $\sigma$ of $\mathbf{R}^{n}$ which fixes the $n$-th standard basis vector. In particular, for any two points $z, z^{\prime} \in \mathbf{H}^{n}$, the value of the expression $\operatorname{Im} z / \operatorname{Im} z^{\prime}$ depends only on the point $\zeta$ and not on the choice of $J$. 
Proposition. With the above conventions we have

$$
\mathcal{P}\left(z, z^{\prime}, \zeta\right)=\operatorname{Im} z^{\prime} / \operatorname{Im} z
$$

for any points $z, z^{\prime} \in \mathbf{H}^{n}$.

Proof. We identify $\mathbf{H}^{n}$ with $\mathbf{R}^{n-1} \times(0, \infty)$ via the diffeomorphism $J$, and write $z=(x, t)$ and $z^{\prime}=\left(x^{\prime}, t^{\prime}\right)$, where $x, x^{\prime} \in \mathbf{R}^{n-1}$ and $t, t^{\prime}>0$. We define two sequences $\left(w_{j}\right)_{j \geq 1}$ and $\left(w_{j}^{\prime}\right)_{j \geq 1}$ of points of $\mathbf{H}^{n}$ by $w_{j}=(x, j)$ and $w_{j}^{\prime}=\left(x^{\prime}, j\right)$. For each $j$, the vertical line segments $z w_{j}$ and $z^{\prime} w_{j}^{\prime}$ are hyperbolic geodesic arcs; hence $\operatorname{dist}\left(z, w_{j}\right)=\log \left|j-z_{n}\right|$ and $\operatorname{dist}\left(z^{\prime}, w_{j}^{\prime}\right)=\log \left|j-z_{n}^{\prime}\right|$. On the other hand, the horizontal line segment from $w_{j}$ to $w_{j}^{\prime}$ is an arc of hyperbolic length $1 / j$, and hence $\lim _{j \rightarrow \infty} \operatorname{dist}\left(w_{j}, w_{j}^{\prime}\right)=0$. From the definition of $\mathcal{P}$ we then have

$$
\begin{aligned}
\mathcal{P}\left(z, z^{\prime}, \zeta\right) & =\lim _{j \rightarrow \infty} \exp \left(\operatorname{dist}\left(z, w_{j}\right)-\operatorname{dist}\left(z^{\prime}, w_{j}\right)\right) \\
& =\lim _{j \rightarrow \infty} \exp \left(\operatorname{dist}\left(z, w_{j}\right)-\operatorname{dist}\left(z^{\prime}, w_{j}^{\prime}\right)\right) \\
& =t^{\prime} / t=\operatorname{Im} z^{\prime} / \operatorname{Im} z .
\end{aligned}
$$

The function $\mathcal{P}$ is our version of the Poisson kernel. If $\mathbf{H}^{n}$ is identified with the Poincaré model via an isometry which maps a given point $z_{0}$ to the origin, then the hyperbolic Poisson kernel $P: B^{n} \times S_{\infty} \rightarrow \mathbf{R}$ discussed, for example, in $[\mathrm{N}]$ or $[\mathrm{P}]$ is given by

$$
P(z, \zeta)=\mathcal{P}\left(z_{0}, z, \zeta\right)
$$

This follows from the proposition above together with the calculation [N, Theorem 5.1.3] that if $V$ is the isometry from the Poincaré model to the upper half-space model which sends the origin to the point $(0,1) \in \mathbf{R}^{n} \times(0, \infty)$ and maps $\zeta$ to $\infty$ then $P\left(V^{-1}(z), \zeta\right)=\operatorname{Im} z$.

2.4. Conformal expansion factors. Consider a point $z \in \mathbf{H}^{n}$ and an isometry $\gamma: \mathbf{H}^{n} \rightarrow$ $\mathbf{H}^{n}$. By 1.1, $\gamma$ induces a conformal diffeomorphism $\gamma_{\infty}$ of $S_{\infty}$. If we equip $S_{\infty}$ with the round metric centered at $z$, then at each point $\zeta \in S_{\infty}$, the map $\gamma_{\infty}$ has a well-defined conformal expansion factor $\lambda>0$. (This means that the tangent map $d \gamma_{\infty}: T_{\xi}\left(S_{\infty}\right) \rightarrow$ $T_{\gamma_{\infty}(\xi)}\left(S_{\infty}\right)$ satisfies $\left|d \gamma_{\infty}(v)\right|=\lambda|v|$ for every tangent vector $v$ at $\zeta$, where $|\cdot|$ denotes length in the round metric.) We shall denote the conformal expansion factor of $\gamma_{\infty}$ at $\zeta$ by $\lambda_{\gamma, z}(\zeta)$. Thus $\lambda_{\gamma, z}$ is a smooth positive-valued function on $S_{\infty}$.

Note that if $\zeta$ is a fixed point of $\gamma$ then $d \gamma_{\infty}(\zeta)$ is a linear automorphism of $T_{\xi}\left(S_{\infty}\right)$ and we have $\lambda_{\gamma, z}(\zeta)=\left|\operatorname{det} d \gamma_{\infty}(\zeta)\right|^{1 /(n-1)}$. Thus in this case $\lambda_{\gamma, z}(\zeta)$ may be calculated without reference to the round metric or the point $z$.

Proposition. Let $z$ be any point of $\mathbf{H}^{n}$ and let $\gamma$ be any isometry of $\mathbf{H}^{n}$. Then we have $\lambda_{\gamma, z}(\zeta)=\mathcal{P}\left(z, \gamma^{-1} z, \zeta\right)$ for every point $\zeta \in S_{\infty}$.

Proof. The corresponding statement for the Poincaré model is given in [N, Lemma 3.4.2]. This together with 2.2 gives the proposition. 
2.5. The Laplacian. For any Riemannian $n$-manifold $M$ we have a Laplacian operator

$$
\Delta=* d * d: C^{\infty}(M) \rightarrow C^{\infty}(M)
$$

where $*$ is the Hodge star and $d$ denotes exterior differentiation.

Using Proposition 2.3, one shows the following by a straightforward calculation in the upper half-space model. (See [N, Theorem 5.1.3]).

Proposition. For any $z \in \mathbf{H}^{n}$, and $\zeta \in S_{\infty}$ and any real number $r$, the function $\phi_{\zeta}$ : $\mathbf{H}^{n} \rightarrow \mathbf{R}$ defined by $\phi_{\zeta}(z)=\mathcal{P}\left(z_{0}, z, \zeta\right)^{r}$ satisfies the equation $\Delta \phi_{\zeta}=-r(n-r-1) \phi_{\zeta}$.

Proposition 2.5 is the starting point for the use of the Poisson kernel in constructing solutions to the equation $\Delta \phi_{\zeta}=-r(n-r-1) \phi_{\zeta}$ ("generalized eigenfunctions" for the Laplacian) with prescribed "boundary values." From the point of view of the present paper this construction is most naturally described in terms of the notion, first formalized by Sullivan, of a "conformal density." We shall discuss this in the next section.

\section{§3. Conformal Densities}

In this section we discuss a $\Gamma$-equivariant potential theory in hyperbolic space, where $\Gamma$ is a Kleinian group. Our treatment, which roughly parallels the one given in Nicholls's recent book $[\mathrm{N}]$, is formulated in terms of Sullivan's notion of a conformal density. This makes the equivariant theory work out quite neatly. Our approach to the uniqueness of a $\Gamma$-invariant conformal density when all $\Gamma$-invariant super-harmonic functions are constant is somewhat different from the approach used in $[\mathrm{N}]$ and [Su1]. We show (Proposition 3.7) that an arbitrary $(n-1)$-conformal density on $\mathbf{H}^{n}$, not necessarily $\Gamma$-invariant, is determined by the associated harmonic funtion. This works only when the degree of the conformal density is $n-1$, but is simpler than the arguments in $[\mathrm{N}]$ and [Su1] which depend on the ergodicity of the action of $\Gamma$ on its limit set with respect to the measure class determined by the conformal density.

3.1. By a Borel measure on a locally compact metrizable space $X$ we shall mean a measure on the Borel subsets of $X$ which is finite on the compact subsets of $X$. Measures will always be understood to be non-negative: we shall have no occasion to consider signed measures. If $\mu$ is a finite measure on a space $X$, the number $\mu(X)$ will be called the total mass of $\mu$. If $\mu$ is a measure, and $f$ a measurable function, on a measurable space $(X, \mathcal{B})$, and if $\nu$ is the measure defined by $\nu(E)=\int_{E} f d \mu$ for every $E \in \mathcal{B}$, then we write $d \nu=f d \mu$.

If $x$ is a point of a locally compact metrizable space $X$, we denote by $\delta_{x}$ the Dirac measure supported at $x$ : by definition, for any Borel set $E \subset X$ we have $\delta_{x}(E)=1$ if $x \in E$ and $\delta_{x}(E)=0$ otherwise.

If $X$ and $Y$ are locally compact metrizable spaces and $f: X \rightarrow Y$ is a homeomorphism, then any Borel measure $\mu$ on $Y$ gives rise to a measure on $X$, called the pull-back of $\mu$ and denoted $f^{*} \mu$. It is defined by $\left(f^{*} \mu\right)(E)=\mu(f(E))$ for any Borel set $E \subset X$.

3.2. Conformal densities. Let $n$ be an integer $\geq 2$, and let $D$ be a number in $[0, n-1]$. By a $D$-conformal density for the sphere at infinity $S_{\infty}^{n-1} \subset \overline{\mathbf{H}}^{n}$ we mean a family $\mathcal{M}=\left(\mu_{z}\right)_{z \in \mathbf{H}^{n}}$ 
of finite Borel measures on $S_{\infty}=S_{\infty}^{n-1}$, indexed by the points of $\mathbf{H}^{n}$, such that for any two points $z, z^{\prime} \in \mathbf{H}^{n}$ we have $d \mu_{z^{\prime}}=\mathcal{P}\left(z, z^{\prime}, \cdot\right)^{D} d \mu_{z}$.

A family $\mathcal{M}=\left(\mu_{z}\right)_{z \in \mathbf{H}^{n}}$ of finite Borel measures on $S_{\infty}$ will be called a conformal density if it is a $D$-conformal density for some $D \in[0, n-1]$.

The trivial conformal density is $(0)_{z \in \mathbf{H}^{n}}$; it will be denoted by 0 . Note that 0 is a $D$ conformal density for every $D \in[0, n-1]$. On the other hand, it is clear that a non-trivial conformal density $\mathcal{M}$ can be a $D$-conformal density for only one value of $D$; we call $D$ the degree of $\mathcal{M}$.

If $z_{0}$ is a point of $\mathbf{H}^{n}$ and $\mu$ is a finite Borel measure on $S_{\infty}$, then for any $D \geq 0$ there is a unique $D$-conformal density $\mathcal{M}=\left(\mu_{z}\right)$ such that $\mu_{z_{0}}=\mu$. Indeed, uniqueness is clear since for any $z \in \mathbf{H}^{n}$ we must have $d \mu_{z}=\mathcal{P}\left(z_{0}, z, \cdot\right)^{D} d \mu$. To prove existence, we define $\mu_{z}$, for each $z \in \mathbf{H}^{n}$, to be the unique Borel measure such that $d \mu_{z}=\mathcal{P}\left(z_{0}, z, \cdot\right)^{D} d \mu$; using 2.3 we find that for any points $z, z^{\prime} \in \mathbf{H}^{n}$ we have

$$
\mathcal{P}\left(z_{0}, z^{\prime}, \cdot\right)^{D}=\mathcal{P}\left(z_{0}, z, \cdot\right)^{D} \mathcal{P}\left(z, z^{\prime}, \cdot\right)^{D}
$$

and hence

$$
d \mu_{z^{\prime}}=\mathcal{P}\left(z_{0}, z^{\prime}, \cdot\right)^{D} d \mu=\mathcal{P}\left(z_{0}, z, \cdot\right)^{D} \mathcal{P}\left(z, z^{\prime}, \cdot\right)^{D} d \mu=\mathcal{P}\left(z, z^{\prime}, \cdot\right)^{D} d \mu_{z},
$$

so that $\left(\mu_{z}\right)$ is the required conformal density.

If $\mathcal{M}=\left(\mu_{z}\right)$ is a conformal density then all the measures $\mu_{z}, z \in \mathbf{H}^{n}$, have the same support; this follows from the definition, since the function $\mathcal{P}\left(z, z^{\prime}, \cdot\right)$ is strictly positive on $S_{\infty}$ for all $z, z^{\prime} \in \mathbf{H}^{n}$. The common support of the $\mu_{z}$ will be called the support of $\mathcal{M}$ and will be denoted $\operatorname{supp} \mathcal{M}$.

If $\mathcal{M}=\left(\mu_{z}\right)_{z \in \mathbf{H}^{n}}$ and $\mathcal{M}^{\prime}=\left(\mu_{z}^{\prime}\right)_{z \in \mathbf{H}^{n}}$ are two $D$-conformal densities for a given $D$, it is clear that $\left(\mu_{z}+\mu_{z}^{\prime}\right)_{z \in \mathbf{H}^{n}}$ is also a conformal density; it will be denoted $\mathcal{M}+\mathcal{M}^{\prime}$. More generally we can define the sum $\sum_{i=}^{n} \mathcal{M}_{i}$ of any finite family $\left(\mathcal{M}_{i}\right)_{1 \leq i \leq n}$ of $D$-conformal densities.

Now let $\mathcal{M}=\left(\mu_{z}\right)_{z \in \mathbf{H}^{n}}$ be a $D$-conformal density and $f$ a continuous positive-valued function on $S_{\infty}$. For each $z \in \mathbf{H}^{n}$ let $\mu_{z}^{\prime}$ be the Borel measure defined by $d \mu_{z}^{\prime}=f d \mu_{z}$. It is clear that $\mathcal{N}=\left(\mu_{z}^{\prime}\right)_{z \in \mathbf{H}^{n}}$ is a $D$-conformal density; we shall indicate that $\mathcal{N}$ is defined in this way from $f$ and $\mathcal{M}$ by writing $d \mathcal{N}=f d \mathcal{M}$.

3.3. The area density. For any point $z \in \mathbf{H}^{n}$, the round metric centered at $z$ determines an area measure on $S_{\infty}$. We let $A_{z}$ denote such an area measure normalized so as to have total mass 1. It follows from Proposition 2.4 and the change-of-variable formula for the Lebesgue integral that $\left(A_{z}\right)_{z \in \mathbf{H}^{n}}$ is an $(n-1)$-conformal density. This will be called the area density and will be denoted $\mathcal{A}$.

3.4. Invariant Conformal Densities. Suppose that $\mathcal{M}=\left(\mu_{z}\right)$ is a $D$-conformal density and that $\gamma: \mathbf{H}^{n} \rightarrow \mathbf{H}^{n}$ is an isometry. Then the family of Borel measures $\left(\gamma_{\infty}^{*} \mu_{\gamma z}\right)_{z \in \mathbf{H}^{n}}$ is also a $D$-conformal density. Indeed, for any points $z, z^{\prime} \in \mathbf{H}^{n}$ and any continuous function 
$f$ on $S_{\infty}$, we have

$$
\begin{aligned}
\int f d\left(\gamma_{\infty}^{*} \mu_{\gamma z^{\prime}}\right) & =\int\left(f \circ \gamma_{\infty}^{-1}\right) d \mu_{\gamma z^{\prime}} \\
& =\int f \circ \gamma_{\infty}^{-1}(w) \mathcal{P}\left(\gamma z, \gamma z^{\prime}, w\right)^{D} d \mu_{\gamma z}(w) \\
& =\int f \circ \gamma_{\infty}^{-1}(w) \mathcal{P}\left(z, z^{\prime}, \gamma^{-1} w\right)^{D} d \mu_{\gamma z}(w) \\
& =\int f \mathcal{P}\left(z, z^{\prime}, \cdot\right)^{D} d\left(\gamma_{\infty}^{*} \mu_{\gamma z}\right) .
\end{aligned}
$$

We call $\left(\gamma_{\infty}^{*} \mu_{\gamma z}\right)$ the pull-back of the conformal density $\mathcal{M}$, and we shall denote it by $\gamma^{*} \mathcal{M}$.

Now let $\Gamma$ be any group of isometries of $\mathbf{H}^{n}$. A conformal density $\mathcal{M}$ will be termed $\Gamma$-invariant if we have $\gamma^{*} \mathcal{M}=\mathcal{M}$ for every $\gamma \in \Gamma$. The area density is clearly invariant under the full group of isometries of $\mathbf{H}^{n}$.

If a $D$-conformal density $\mathcal{M}=\left(\mu_{z}\right)$ is $\Gamma$-invariant then for any $z \in \mathbf{H}^{n}$ and any $\gamma \in \Gamma$ we have $d\left(\gamma^{*} \mu_{z}\right)=d \mu_{\gamma^{-1} z}=\mathcal{P}\left(z, \gamma^{-1} z, \cdot\right)^{D} \cdot d \mu_{z}$; hence in view of 2.4 we have

$$
d\left(\gamma^{*} \mu_{z}\right)=\lambda_{\gamma, z}^{D} d \mu_{z}
$$

3.5. Eigenfunctions of the Laplacian. Let $n \geq 2$ be an integer, and let $D$ be a number in the interval $[0, n-1]$. Let $\mathcal{M}=\left(\mu_{z}\right)_{z \in \mathbf{H}^{n}}$ be a $D$-conformal density. Let us consider the non-negative-valued function $u=u_{\mathcal{M}}$ on $\mathbf{H}^{n}$ defined by $u(z)=\mu_{z}\left(S_{\infty}\right)$. If we fix any point $z_{0} \in \mathbf{H}^{n}$, and set $\mu=\mu_{z_{0}}$, then according to the definition of a conformal density, $u$ may be written as

$$
u(z)=\int_{S_{\infty}} \mathcal{P}\left(z_{0}, z, \zeta\right)^{D} d \mu(\zeta)
$$

For the area density $\mathcal{A}$ we clearly have $u_{\mathcal{A}} \equiv 1$.

According to Proposition 2.5, for any $\zeta \in S_{\infty}$ the function $\phi_{\zeta}=\mathcal{P}\left(z_{0}, \cdot, \zeta\right)^{D}$ is $C^{\infty}$ and satisfies $\Delta \phi_{\zeta}=-D(n-D-1) \phi_{\zeta}$. By differentiating under the integral sign in (3.5.1) it follows that for any $D$-conformal density $\mathcal{M}$ the function $u=u_{\mathcal{M}}$ is $C^{\infty}$ and satisfies the equation

$$
\Delta u=-D(n-D-1) u \text {. }
$$

In particular, if $D=n-1$ then (3.5.2) says that $u$ is harmonic.

If $\mathcal{M}$ is invariant (3.4) under a group $\Gamma$ of isometries of $\mathbf{H}^{n}$, then for any $\gamma \in \Gamma$ and any $z \in \mathbf{H}^{n}$ we have

$$
u(\gamma z)=\mu_{\gamma z}\left(S_{\infty}\right)=\left(\gamma^{*} \mu_{z}\right)\left(S_{\infty}\right)=\mu_{z}\left(S_{\infty}\right)=u(z)
$$

Thus $u$ is $\Gamma$-invariant (i.e. constant on the orbits of $\Gamma$.) 
3.6. Proposition. Let $f$ be a continuous function on $S_{\infty}$ and let $\mathcal{N}$ be the conformal density defined by $d \mathcal{N}=f d \mathcal{A}$, where $\mathcal{A}$ is area density. Then the function $\bar{u}: \overline{\mathbf{H}}^{n} \rightarrow \mathbf{R}$, defined by $\bar{u} \mid \mathbf{H}^{n}=u_{\mathcal{N}}$ and $\bar{u} \mid S_{\infty}=f$, is continuous.

Proof. We identify $\mathbf{H}^{n}$ via a conformal diffeomorphism with the Poincaré model. Thus $S_{\infty}$ is identified with the unit sphere $S^{n-1}$. We write $A=A_{0}$. Since $f$ is continuous and $u=u_{\mathcal{N}}$ is $C^{\infty}$, we need only show that if $\left(z_{i}\right)$ is a sequence of points in $\mathbf{H}^{n}$ converging to a point $\zeta_{0} \in S_{\infty}$, then $u\left(z_{i}\right)$ converges to $f\left(\zeta_{0}\right)$. We set $C=f\left(\zeta_{0}\right)$. For each $i$ we have

$$
u\left(z_{i}\right)=\int_{S_{\infty}} \mathcal{P}\left(0, z_{i}, \zeta\right)^{n-1} f(\zeta) d A(\zeta) .
$$

On the other hand, the integral $\int_{S_{\infty}} \mathcal{P}\left(0, z_{i}, \zeta\right)^{n-1} d A(\zeta)$ represents the total mass of $A_{z_{i}}$, which is 1 . Hence

$$
u\left(z_{i}\right)-C=\int_{S_{\infty}} \mathcal{P}\left(0, z_{i}, \zeta\right)^{n-1}(f(\zeta)-C) d A(\zeta) .
$$

To show that $u\left(z_{i}\right) \rightarrow C$, it suffices to show that $\lim \sup \left|u\left(z_{i}\right)-C\right|<\epsilon$ for any prescribed $\epsilon>0$. Given $\epsilon$, let $U$ be a neighborhood of $\zeta_{0}$ in $S_{\infty}$ such that $|f(\zeta)-C|<\epsilon$ for every $\zeta \in U$. We may take $U$ to be the $\delta$-neighborhood for some $\delta>0$ in the standard metric on $S^{n-1}$; thus a point $\zeta$ lies in $U$ if and only if $\angle\left(\zeta_{0}, 0, \zeta\right)<\delta$. We may write $u\left(z_{i}\right)-C$ as

$$
\int_{U} \mathcal{P}\left(0, z_{i}, \zeta\right)^{n-1}(f(\zeta)-C) d A(\zeta)+\int_{S_{\infty}-U} \mathcal{P}\left(0, z_{i}, \zeta\right)^{n-1}(f(\zeta)-C) d A(\zeta)
$$

The first term in the above expression is bounded above in absolute value by $\epsilon$. We shall complete the proof by showing that the second term tends to 0 as $i \rightarrow \infty$.

Since $f$ is continuous on $S_{\infty}$, it is enough to show that $\sup _{\zeta \in S_{\infty}-U} \mathcal{P}\left(0, z_{i}, \zeta\right)$ tends to 0 as $i \rightarrow \infty$. This follows easily from the formula (2.1.1) for $\mathcal{P}$.

3.7. Proposition. If $\mathcal{M}$ and $\mathcal{M}^{\prime}$ are $(n-1)$-conformal densities for $\mathbf{H}^{n}$ such that $u_{\mathcal{M}}=$ $u_{\mathcal{M}^{\prime}}$, then $\mathcal{M}=\mathcal{M}^{\prime}$.

Proof. For the purpose of this argument we identify $\mathbf{H}^{n}$ with the Poincare model $B^{n}$, so that $\overline{\mathbf{H}}^{n}$ is identified with $\bar{B}^{n}$. Then any point in $\mathbf{H}^{n}$ may be written in the form $t \theta$, where $\theta \in S^{n-1}$ and $0 \leq t<1$.

We will use the following fundamental symmetry of the function $\mathcal{P}$ which follows directly from the formula (2.1.1):

$$
\mathcal{P}(0, t \zeta, \theta)=\mathcal{P}(0, t \theta, \zeta)
$$

for any $t \in[0,1)$ and any $\zeta, \theta \in S^{n-1}$.

Now let $\mathcal{M}=\left(\mu_{z}\right)$ be any $(n-1)$-conformal density and let $f$ be any continuous function on $S_{\infty}$. Let $\mathcal{N}$ be the conformal density defined by $d \mathcal{N}=f d \mathcal{A}$, where $\mathcal{A}$ is the area density. By Proposition 3.6 the function $\bar{u}: \overline{\mathbf{H}}^{n} \rightarrow \mathbf{R}$, defined by $\bar{u} \mid \mathbf{H}^{n}=u_{\mathcal{N}}$ and $\bar{u} \mid S_{\infty}=f$, is continuous. Hence

$$
\int f d \mu_{0}=\lim _{t \rightarrow 1^{-}} \int u_{\mathcal{N}}(t \zeta) d \mu_{0}(\zeta)
$$


For any $t<1$ we have, by (3.7.1) and the Fubini Theorem,

$$
\begin{aligned}
\int u_{\mathcal{N}}(t \zeta) d \mu_{0}(\zeta) & =\iint f(\theta) \mathcal{P}(0, t \zeta, \theta)^{n-1} d A(\theta) d \mu_{0}(\zeta) \\
& =\iint \mathcal{P}(0, t \theta, \zeta)^{n-1} d \mu_{0}(\zeta) f(\theta) d A(\theta)=\int u_{\mathcal{M}}(t \theta) f(\theta) d A(\theta)
\end{aligned}
$$

If $\mathcal{M}^{\prime}=\left(\mu_{z}^{\prime}\right)$ is a second conformal density such that $u_{\mathcal{M}}=u_{\mathcal{M}^{\prime}}$, then for any $t<1$ we have

$$
\int u_{\mathcal{N}}(t \zeta) d \mu_{0}^{\prime}(\zeta)=\int u_{\mathcal{M}^{\prime}}(t \theta) f(\theta) d A(\theta)=\int u_{\mathcal{M}}(t \theta) f(\theta) d A(\theta)=\int u_{\mathcal{N}}(t \zeta) d \mu_{0}(\zeta) .
$$

Taking limits as $t \rightarrow 1$, it follows by (3.7.2) that

$$
\int f d \mu_{0}=\int f d \mu_{0}^{\prime}
$$

for every continuous function $f$ on $S_{\infty}$. Hence $\mu_{0}=\mu_{0}^{\prime}$. By 3.2 it follows that $\mathcal{M}=\mathcal{M}^{\prime}$.

3.8. We shall need the following elementary result concerning the degree of a $\Gamma$-invariant conformal density, when $\Gamma$ is a discrete group (cf. [N, Corollary 3.4.5]).

Proposition. Let $\Gamma$ be a non-elementary discrete group of isometries of $\mathbf{H}^{n}$. Then every non-trivial $\Gamma$-invariant conformal density for $\mathbf{H}^{n}$ has strictly positive degree.

Proof. Suppose that there exists a non-trivial $\Gamma$-invariant conformal density of degree 0. By definition, this means that there is a non-zero $\Gamma$-invariant finite Borel measure $\mu$ on $S_{\infty}$.

Since $\Gamma$ is non-elementary, some element $\gamma$ of $\Gamma$ is loxodromic; let $P$ and $Q$ be the fixed points of $\gamma_{\infty}$. Then any point $\zeta \in S_{\infty}-\{P, Q\}$ has a neighborhood $U$ in $S_{\infty}$ such that $\gamma_{\infty}^{k}(U) \cap U=\emptyset$ for all $k>0$. Since $\mu$ is finite and countably additive, it follows that $\mu(U)=0$. This shows that supp $\mu \subset\{P, Q\}$. But supp $\mu$ is non-empty and invariant under $\Gamma$; thus $S_{\infty}$ contains a $\Gamma$-invariant subset of cardinality 1 or 2 . Since $\Gamma$ is discrete, it follows that $\Gamma$ has an abelian subgroup of finite index. This contradicts the hypothesis that $\Gamma$ is non-elementary, and the proposition is proved.

3.9. A $C^{\infty}$ function $f$ on a Riemannian manifold is termed superharmonic if it satisfies the inequality $\Delta f \leq 0$.

Proposition. Let $\Gamma$ be a non-elementary discrete group of isometries of $\mathbf{H}^{n}$. Suppose that every $\Gamma$-invariant positive-valued superharmonic function on $\mathbf{H}^{n}$ is constant. Then any $\Gamma$-invariant conformal density for $\mathbf{H}^{n}$ is a constant multiple of the area density.

Proof. Suppose that $\mathcal{M}=\left(\mu_{z}\right)$ is a $\Gamma$-invariant $D$-conformal density for some $D \in[0, n-1]$. Since $\Gamma$ is non-elementary, it follows from Proposition 3.9 that $D \neq 0$. We may assume that $\mathcal{M} \neq 0$, so that the function $u=u_{\mathcal{M}}$ is positive-valued. By $3.5 u$ is $\Gamma$-invariant and satisfies the equation $\Delta u=-D(n-D-1) u$; in particular $u$ is superharmonic. Hence by the hypothesis $u$ is a constant $C$. 
Thus $0=\Delta u \equiv D(n-D-1) u$. Since $D \neq 0$ it follows that $D=n-1$. Since $u_{\mathcal{M}}=C=u_{C \mathcal{A}}$, Proposition 3.7 then guarantees that $\mathcal{M}=C \mathcal{A}$.

Note that our approach to this uniqueness question is based on Proposition 3.7, which applies to an arbitrary $(n-1)$-conformal density. The support of the conformal density $\mathcal{M}$ plays no role in the argument.

Another, somewhat less elementary, approach is found in the literature. Suppose that $\mathcal{M}=\left(\mu_{z}\right)$ is a $\Gamma$-invariant $D$-conformal density with $\operatorname{supp} \mathcal{M}=\Lambda_{\Gamma}$. (A construction, due to Patterson, of conformal densities with these properties will be discussed in the next section.) Then any $\Gamma$-invariant $D$-conformal density supported on $\Lambda_{\Gamma}$ is a constant multiple of $\mathcal{M}$ provided that $\Gamma$ acts ergodically on $S_{\infty}$ with respect to the measure class determined by $\mathcal{M}\left[\mathrm{N}\right.$, Theorem 4.2.1]. Suppose that $A$ is a $\Gamma$-invariant subset of $S_{\infty}$ such that $A$ and $S_{\infty}-A$ are sets of positive measure with respect to this measure class. Let $\mathcal{M}^{\prime}$ be given by $d \mathcal{M}^{\prime}=\chi_{A} d \mathcal{M}$. Using points of density one can show that the ratio $u_{\mathcal{M}^{\prime}} / u_{\mathcal{M}}$ tends to $\chi_{A}$ along almost all $\left(\mu_{0}\right)$ rays from the origin in the Poincaré model. Given this, the ergodicity follows if one knows that the ratio of any two positive $\Gamma$-invariant eigenfunctions of $\Delta$, with eigenvalue $-D(n-D-1)$, is bounded above and below by positive constants. In [Su3] Sullivan uses this method to give examples of Kleinian groups $\Gamma$, for which there exist unique $\Gamma$-invariant conformal densities of degree 2 supported on $\Lambda_{\Gamma}$, but where these conformal densities are not constant multiples of the area density. (In fact, for these groups $\Lambda_{\Gamma}$ has area 0 , although it follows from the existence of such conformal densities that $\Lambda_{\Gamma}$ has Hausdorff dimension 2.)

\section{$\S 4$. The Patterson Construction}

In this section we describe a construction first introduced by Patterson $[\mathrm{P}]$, and extensively studied by Sullivan ([Su1], [Su2], [Su3]), for associating to a Kleinian group $\Gamma$ a $\Gamma$-invariant conformal density supported on the limit set of $\Gamma$. By taking a slightly more general point of view than Patterson's, we are able to construct decompositions of a Patterson density corresponding to decompositions of the group. In Section 5, by specializing to the case of a free group, we shall obtain the paradoxical decomposition described in the introduction.

4.1. Uniformly discrete sets. A subset $W$ of $\mathbf{H}^{n}$ will be called uniformly discrete if there is a number $\epsilon>0$ such that $\operatorname{dist}(z, w)>\epsilon$ for any two distinct points $z, w \in W$. Such a number $\epsilon$ will be called a modulus of discreteness for $W$.

If $\Gamma$ is any discrete group of isometries of $\mathbf{H}^{n}$, then for any $w \in \mathbf{H}^{n}$, the orbit $W=\Gamma w$ is a uniformly discrete set. (Indeed, since $\Gamma$ is discrete and $w$ has compact stabilizer in the isometry group of $\mathbf{H}^{n}$, the orbit $\Gamma w$ is discrete. Thus there is a number $\epsilon>0$ such that $\operatorname{nbhd}_{\epsilon}(w) \cap \Gamma w=\{w\}$. Since $\Gamma$ consists of isometries it follows that $\epsilon$ is a modulus of discreteness.)

If $W \subset \mathbf{H}^{n}$ is any uniformly discrete set, the closure of $W$ in $\overline{\mathbf{H}}^{n}$ has the form $W \cup \Lambda$, where $\Lambda$ is a closed subset of $S_{\infty}$. We call $\Lambda$ the limit set of $W$. Note that $\Lambda$ is empty if and only if $W$ is finite.

If $\Gamma$ is a discrete group of isometries, the limit set of any orbit of $\Gamma$ is the limit set of $\Gamma$. 
4.2. The main result of this section is:

Proposition. Let $W$ be an infinite, uniformly discrete subset of $\mathbf{H}^{n}$, and let $\mathfrak{V}$ be a countable collection of subsets of $W$. Suppose that $W \in \mathfrak{V}$. Then there exist a number $D \in[0, n-1]$ and a family $\left(\mathcal{M}_{V}\right)_{V \in \mathfrak{V}}$ of $D$-conformal densities for $\mathbf{H}^{n}$, indexed by the collection $\mathfrak{V}$, satisfying the following conditions.

(i) $\mathcal{M}_{W} \neq 0$.

(ii) For any finite family $\left(V_{i}\right)_{1 \leq i \leq m}$ of disjoint sets in $\mathfrak{V}$ such that $V=\coprod_{i=1}^{m} V_{i} \in \mathfrak{V}$, we have $\mathcal{M}_{V}=\sum_{i=1}^{m} \mathcal{M}_{V_{i}}$.

(iii) For any $V \in \mathfrak{V}$ and any isometry $\gamma: \mathbf{H}^{n} \rightarrow \mathbf{H}^{n}$ such that $\gamma V \in \mathfrak{V}$, we have $\gamma_{\infty}^{*}\left(\mathcal{M}_{\gamma V}\right)=\mathcal{M}_{V}$.

(iv) For any $V \in \mathfrak{V}$, the support of $\mathcal{M}_{V}$ is contained in the limit set of $\mathcal{M}_{V}$. In particular, for any finite set $V \in \mathfrak{V}$ we have $\mathcal{M}_{V}=0$.

Before turning to the proof of the Proposition, let us point out one of its consequences. If $\Gamma$ is any non-elementary discrete group of isometries of $\mathbf{H}^{n}$, then any orbit $W$ of $\Gamma$ is uniformly discrete by 4.1 and infinite because $\Gamma$ is non-elementary. Thus we may apply Proposition 4.2 to $W$, taking $\mathfrak{V}$ to be any countable collection of subsets of $W$ with $W \in \mathfrak{V}$ (for example $\mathfrak{V}=\{W\}$ ). This gives a conformal density $\mathcal{M}=\mathcal{M}_{W}$. It follows from 4.2(iii) that $\mathcal{M}$ is $\Gamma$ - invariant. In particular the support of $\mathcal{M}$ is $\Gamma$-invariant. On the other hand, we have $\operatorname{supp} \mathcal{M} \neq \emptyset$ by $4.2(\mathrm{i})$; and by $4.2(\mathrm{iv})$ we have $\operatorname{supp} \mathcal{M} \subset \Lambda_{\Gamma}$. Since the action of $\Gamma$ on $\Lambda_{\Gamma}$ is topologically minimal, it follows that $\operatorname{supp} \mathcal{M}=\Lambda_{\Gamma}$.

In particular, taking $\mathfrak{V}=\{W\}$, we obtain the following.

Corollary. (Patterson $[P]$ ) If $\Gamma$ is any non-elementary discrete subgroup of isometries of $\mathbf{H}^{n}$, there is a $\Gamma$-invariant conformal density for $\mathbf{H}^{n}$ whose support is $\Lambda_{\Gamma}$.

The rest of this section is devoted to the proof of the proposition. We are given an infinite, uniformly discrete set $W \subset \mathbf{H}^{n}$ and a countable collection $\mathfrak{V}$ of subsets of $W$, with $W \in \mathfrak{V}$.

4.3. The Poincaré Series. For any point $z \in \mathbf{H}^{n}$ and any $s \geq 0$ we denote by $\Sigma(z, s)$ the sum of the "Poincaré series" for the uniformly discrete set $W$. That is,

$$
\Sigma(z, s)=\sum_{w \in W} e^{-s \operatorname{dist}(w, z)}
$$

where we interpret the sum as a non-negative number or $+\infty$.

The following properties of $\Sigma(z, s)$ are immediate from the definition.

4.3.1. For any $z \in \mathbf{H}^{n}$ and any $s \geq s^{\prime} \geq 0$ we have $\Sigma\left(z, s^{\prime}\right) \geq \Sigma(z, s)$.

4.3.2. For any $z, z^{\prime} \in \mathbf{H}^{n}$ and any $s \geq 0$ we have

$$
\Sigma\left(z^{\prime}, s\right) \leq e^{s \operatorname{dist}\left(z, z^{\prime}\right)} \Sigma(z, s)
$$


Lemma. There is a unique non-negative number $D \leq n-1$ such that, for every point $z \in \mathbf{H}^{n}$, the sum $\Sigma(z, s)$ is finite for all $s>D$ and infinite whenever $0<s<D$.

Proof. First we note that $\Sigma(z, s)$ is finite for $s>n-1$. This is proved, in the case that $W$ is an orbit of a discrete group, in [N, Theorem 1.6.1]. The proof applies as well to an arbitrary uniformly discrete set. Briefly, the idea is that the Poincaré series converges as long as $s$ is larger than the exponential growth rate of the number $N_{R}$ of points of $W$ in the ball of radius $R$ about $z$. The uniform discreteness of $W$ implies that $N_{R}$ cannot grow faster than the volume $V(R)$ of a ball of radius $R$. However, we have $V(R)=A_{n} \int_{0}^{r} \sinh ^{n-1} x d x$, where $A_{n}$ is a number depending only on the dimension $n$. Hence for all $R$ we have $V(R) \leq B_{n} e^{(n-1) R}$, where $B_{n}$ is again a number depending only on $n$.

It now follows from (4.3.1) and the discussion in 4.3 that for each $z \in \mathbf{H}^{n}$ there is a unique number $D_{z} \leq n-1$ such that $\Sigma(z, s)<\infty$ for all $s>D_{z}$ and $\Sigma(z, s)=\infty$ whenever $0<s<D_{z}$. It follows from 4.3.2 that $D_{z}$ is independent of $z$.

The number $D$ given by the lemma is called the critical exponent of $W$.

4.4. The critically divergent case. The construction of the conformal densities in the statement of Proposition 4.2 is simplest in the case where $\Sigma(z, D)$ is infinite for every $z \in \mathbf{H}^{n}$. We sketch the construction in this case before discussing the general case.

First we define a family of measures $\mu_{V, z, s}$ on $\overline{\mathbf{H}}^{n}$ by

$$
\mu_{V, z, s}=\frac{1}{\Sigma(z, s)} \sum_{w \in V} e^{-s \operatorname{dist}(w, z)} \delta_{w}
$$

for $z \in \mathbf{H}^{n}$ and $s>D$.

Now fix an arbitrary point $z_{0} \in \mathbf{H}^{n}$ and let $\left(s_{j}\right)_{j \geq 0}$ be a decreasing sequence of real numbers converging to $D$ such that the sequence of measures $\left(\mu_{V, s_{j}, z_{0}}\right)$ is a weakly convergent sequence. Let $\mu_{V, z_{0}}$ denote the limit. The condition $\Sigma(z, D)=\infty$ implies that the support of $\mu_{V, z_{0}}$ is contained in $S_{\infty}$.

It is immediate from the definition that for all $s>D$

$$
d \mu_{V, z^{\prime}, s}=\Pi\left(z, z^{\prime}, \cdot\right)^{s} d \mu_{V, z, s} .
$$

This implies first of all that the sequence $\left(\mu_{V, z, s_{j}}\right)$ is weakly convergent for each $z \in \mathbf{H}^{n}$. Furthermore, letting $\mu_{V, z}$ denote the limit of the sequence $\left(\mu_{V, z, s_{j}}\right)$, and viewing these measures as measures on $S_{\infty}$, we obtain the formula

$$
d \mu_{V, z^{\prime}}=\mathcal{P}\left(z, z^{\prime}, \cdot\right)^{D} d \mu_{V, z}
$$

In particular, $\mathcal{M}_{V}=\mu_{V, z}$ is a $D$-conformal density.

4.5. Adjustment functions. The above construction must be modified in the case where $\Sigma(z, D)<\infty$; the idea, invented by Patterson, is to perturb the terms in the sum $\Sigma(z, s)$ to obtain an expression $\Sigma^{\prime}(z, s)$ that is always finite when $s>D$ and infinite when $0 \leq$ $s \leq D$. In our formulation, we show that for a suitably chosen real-valued function $\alpha$ the 
construction sketched above can be carried out with $\operatorname{dist}(z, w)$ replaced by $\alpha(\operatorname{dist}(z, w))$ throughout.

If $\alpha$ is any real-valued function on $[0, \infty)$, then for any point $z \in \mathbf{H}^{n}$ and any $s>0$ we define

$$
\Sigma(\alpha, z, s)=\sum_{w \in W} e^{-s \alpha(\operatorname{dist}(w, z))}
$$

where we again interpret the sum as a non-negative number or $+\infty$. Note that if id denotes the identity function on $[0, \infty)$ then $\Sigma(\mathrm{id}, z, s)=\Sigma(z, s)$ for any $z$ and $s$.

We define an adjustment function to be a $C^{1}$ real-valued function $\alpha$ on $[0, \infty)$ such that (i) $\alpha(0)=0$, (ii) $0 \leq \alpha^{\prime}(t) \leq 1$ for all $t>0$, and (iii) $\lim _{t \rightarrow \infty} \alpha^{\prime}(t)=1$. In particular an adjustment function $\alpha$ must satisfy $0 \leq \alpha(t) \leq t$ for all $t \geq 0$. The identity function on $[0, \infty)$ is obviously an adjustment function.

The following generalizations of 4.3.1 and 4.3.2 hold for any adjustment function $\alpha$.

4.5.1. For any $z \in \mathbf{H}^{n}$ and any $s \geq s^{\prime} \geq 0$ we have $\Sigma\left(\alpha, z, s^{\prime}\right) \geq \Sigma(\alpha, z, s)$.

4.5.2. For any $z, z^{\prime} \in \mathbf{H}^{n}$ and any $s \geq 0$ we have

$$
\Sigma\left(\alpha, z^{\prime}, s\right) \leq e^{s \operatorname{dist}\left(z, z^{\prime}\right)} \Sigma(\alpha, z, s) .
$$

Indeed, 4.5.1 is immediate; and 4.5.2 follows from the observation that, by virtue of condition (ii) in the definition of an adjustment function, we have

$$
\left|\alpha(\operatorname{dist}(w, z))-\alpha\left(\operatorname{dist}\left(w, z^{\prime}\right)\right)\right| \leq\left|\operatorname{dist}(w, z)-\operatorname{dist}\left(w, z^{\prime}\right)\right| \leq \operatorname{dist}\left(z, z^{\prime}\right)
$$

for any $w \in W$.

We also have the following extension of 2.1.

Lemma. For any adjustment function $\alpha$, the function $\bar{\Pi}_{\alpha}: \mathbf{H}^{n} \times \mathbf{H}^{n} \times \overline{\mathbf{H}}^{n} \rightarrow \mathbf{R}$ defined by

$$
\bar{\Pi}_{\alpha}\left(z, z^{\prime}, w\right)=\exp \left(\alpha(\operatorname{dist}(z, w))-\alpha\left(\operatorname{dist}\left(z^{\prime}, w\right)\right)\right)
$$

for $\left(z, z^{\prime}, w\right) \in \mathbf{H}^{n} \times \mathbf{H}^{n} \times \mathbf{H}^{n}$, and

$$
\bar{\Pi}_{\alpha}\left(z, z^{\prime}, \zeta\right)=\mathcal{P}\left(z, z^{\prime}, \zeta\right)
$$

for $\left(z, z^{\prime}, \zeta\right) \in \mathbf{H}^{n} \times \mathbf{H}^{n} \times S_{\infty}$, is continuous.

Proof. It is enough to prove that if $\left(z_{i}\right),\left(z_{i}^{\prime}\right)$ and $\left(w_{i}\right)$ are sequences of points in $\mathbf{H}^{n}$ which converge respectively to points $z \in \mathbf{H}^{n}, z^{\prime} \in \mathbf{H}^{n}$ and $\zeta \in S_{\infty}$, then

$$
\lim _{i \rightarrow \infty} \exp \left(\alpha\left(\operatorname{dist}\left(z_{i}, w_{i}\right)\right)-\alpha\left(\operatorname{dist}\left(z_{i}^{\prime}, w_{i}\right)\right)\right)=\mathcal{P}\left(z, z^{\prime}, \zeta\right) .
$$

By definition, we have

$$
\left.\lim _{i \rightarrow \infty} \exp \left(\operatorname{dist}\left(z_{i}, w_{i}\right)\right)-\operatorname{dist}\left(z_{i}^{\prime}, w_{i}\right)\right)=\mathcal{P}\left(z, z^{\prime}, \zeta\right) .
$$

In particular, $\operatorname{dist}\left(z_{i}, w_{i}\right)-\operatorname{dist}\left(z_{i}^{\prime}, w_{i}\right)$ converges. Since $\alpha^{\prime}(t) \rightarrow 1$ as $t \rightarrow \infty$ we have

$$
\lim _{i \rightarrow \infty} \frac{\alpha\left(\operatorname{dist}\left(z_{i}, w_{i}\right)\right)-\alpha\left(\operatorname{dist}\left(z_{i}^{\prime}, w_{i}\right)\right)}{\operatorname{dist}\left(z_{i}, w_{i}\right)-\operatorname{dist}\left(z_{i}^{\prime}, w_{i}\right)}=1 .
$$

The result follows. 
4.6. Lemma. There exists an adjustment function $\alpha$ such that, for every $z \in \mathbf{H}^{n}$, we have $\Sigma(\alpha, z, s)<\infty$ for all $s>D$, and $\Sigma(\alpha, z, s)=\infty$ whenever $0 \leq s \leq D$.

Proof. First consider an arbitrary adjustment function $\alpha$. Since $\alpha(t) \leq t$ for all $t \geq 0$, we have $\Sigma(\alpha, z, s) \geq \Sigma(z, s)$ for all $s$; hence $\Sigma(\alpha, z, s)=\infty$ when $0 \leq s<D$. Now suppose that $s>D$ and choose a positive number $k<1$ such that $s k<D$. Since $\lim _{t \rightarrow \infty} \alpha^{\prime}(t)=1$, there is a constant $C$ such that $\alpha(t) \geq k t-C$ for all $t \geq 0$. Hence, for $s>D$, we have

$$
\Sigma(\alpha, z, s) \leq e^{C s} \Sigma(z, s k)<\infty .
$$

Thus we need only to construct $\alpha$ so that $\Sigma(\alpha, z, D)=\infty$. We first dispose of the special case $D=0$. Since $W$ is infinite, it is clear that $\Sigma(z, 0)=+\infty$ for every $z$. Hence in the case $D=0$ we may take $\alpha$ to be the identity function.

Now suppose that $D>0$. If $\alpha$ is any adjustment function, it follows from 4.5.2 that $\Sigma(\alpha, z, D)$ is either finite for all $z \in \mathbf{H}^{n}$ or infinite for all $z \in \mathbf{H}^{n}$. Hence we need only show that for a given point $z \in \mathbf{H}^{n}$ there is an adjustment function $\alpha$ such that $\Sigma(\alpha, z, D)=+\infty$.

Let $\left(\theta_{m}\right)_{m \geq 1}$ be a strictly increasing sequence of positive numbers which converge to 1 . For each $m \geq 1$ we set $s_{m}=\theta_{m} D$. Since $0<s_{m}<D$, the sum

$$
\Sigma\left(z, s_{m}\right)=\sum_{w \in W} e^{-s_{m} \operatorname{dist}(w, z)}
$$

is infinite. Hence there is a finite subset $X_{m}$ of $W$ such that

$$
\sum_{w \in X_{m}} e^{-s_{m} \operatorname{dist}(w, z)} \geq m
$$

Let $\left(R_{m}\right)_{m \geq 1}$ be a monotone increasing sequence of positive numbers tending to $+\infty$, such that for each $m \geq 1$ and each $w \in X_{m}$ we have $\operatorname{dist}(z, w) \leq R_{m}$. Let $\beta$ be any continuous, monotone increasing real-valued function on $[0, \infty)$ such that $\beta\left(R_{m}\right)=\theta_{m}$ for all $m>0$ and $\beta(t)<1$ for all $t \geq 0$. Then $\alpha(t)=\int_{0}^{t} \beta(u) d u$ is an adjustment function and $\alpha(t) \leq \theta_{m} t$ whenever $0 \leq t \leq R_{m}$. For each $m>0$ and each $w \in X_{m}$ we have $\operatorname{dist}(w, z)<R_{m}$, and hence

$$
\alpha(\operatorname{dist}(w, z)) D \leq \theta_{m} D \operatorname{dist}(w, z)=s_{m} \operatorname{dist}(w, z) .
$$

Thus

$$
\Sigma(\alpha, z, D) \geq \sum_{w \in X_{m}} e^{-D \alpha(\operatorname{dist}(w, z))} \geq \sum_{w \in X_{m}} e^{-s_{m} \operatorname{dist}(w, z)} \geq m .
$$

Since this holds for all $m \geq 1$, we must have $\Sigma(\alpha, z, D)=+\infty$.

For the rest of this section, we fix an adjustment function $\alpha$ constructed as in the lemma. We shall write $\Sigma^{\prime}(z, s)=\Sigma(\alpha, z, s)$ for all $z$ and $s$. 
4.7. For any $s>D$ and any $V \in \mathfrak{V}$ we have

$$
\sum_{w \in V} e^{-s \alpha(\operatorname{dist}(w, z))} \leq \Sigma^{\prime}(z, s)<\infty
$$

by Lemma 4.6. Hence we may define a Borel measure

$$
\sum_{w \in V} e^{-s \alpha(\operatorname{dist}(w, z))} \delta_{w}
$$

on $\overline{\mathbf{H}}^{n}$. This measure will be denoted $\mu_{V, z, s}$. Its total mass is $\leq \Sigma^{\prime}(z, s)$, with equality when $V=W$.

4.8. For the rest of this section we fix, arbitrarily, a point $z_{0} \in \mathbf{H}^{n}$.

Lemma. There exists a sequence $\left(s_{j}\right)_{j \geq 0}$ of real numbers with the following properties:

(i) $s_{j}>D$ for all $j$, and $\lim _{j \rightarrow \infty} s_{j}=D$;

(ii) for every $V \in \mathfrak{V}$, the sequence $\left(\Sigma^{\prime}\left(z_{0}, s_{j}\right)^{-1} \mu V, z_{0}, s_{j}\right)_{j \geq 0}$ is a weakly convergent sequence of Borel measures on $\overline{\mathbf{H}}^{n}$.

Proof. Let $\left(t_{i}\right)$ be any sequence of numbers $>D$ such that $\lim _{i \rightarrow \infty} t_{i}=D$. For any $V \in \mathfrak{V}$, we have a sequence of measures $\left(\Sigma^{\prime}\left(z_{0}, t_{i}\right)^{-1} \mu_{V, z_{0}, t_{i}}\right)$ which by 4.7 all have total mass $\leq 1$. Given any $V \in \mathfrak{V}$, there is a subsequence $\left(t_{i_{j}}\right)$ such that the sequence $\left(\Sigma^{\prime}\left(z_{0}, t_{i_{j}}\right)^{-1} \mu_{V, z_{0}, t_{i_{j}}}\right)$ is weakly convergent. Since $\mathfrak{V}$ is countable, the conclusion of the lemma follows via diagonalization.

For the rest of the section we fix a sequence $\left(s_{j}\right)$ having the above properties (i) and (ii). For each $j \geq 0$ we set $C_{j}=\Sigma^{\prime}\left(z_{0}, s_{j}\right)$. For each $V \in \mathfrak{V}$ we let $\mu_{V}^{0}$ denote the weak limit of the sequence $\left(\frac{1}{C_{j}} \mu_{V, z_{0}, s_{j}}\right)$; by definition, $\mu_{V}^{0}$ is a Borel measure on $\overline{\mathbf{H}}^{n}$.

4.9. Lemma. We have $C_{j} \rightarrow+\infty$ as $j \rightarrow \infty$.

Proof. By 4.6 we have $\Sigma^{\prime}\left(z_{0}, D\right)=+\infty$. This implies the lemma since the terms of the sum $\Sigma^{\prime}(z, s)=\sum_{i \in I} e^{-s \alpha\left(\operatorname{dist}\left(w_{i}, z\right)\right)}$ are positive-valued continuous functions of $s$.

4.10. Lemma. For each $V \in \mathfrak{V}$, the measure $\mu_{V}^{0}$ has support contained in $S_{\infty}^{n-1}$.

Proof. It is enough to show that $\mu_{V}^{0}(\mathcal{C})=0$ for any compact set $\mathcal{C} \subset \mathbf{H}^{n}$. The set $V \cap \mathcal{C}$ is finite since $V$ is a subset of the uniformly discrete set $W$. We have

$$
\mu_{V, z_{0}, s_{j}}(\mathcal{C})=\sum_{w \in V} \exp \left(-s_{j} \alpha(\operatorname{dist}(w, z))\right) \leq \operatorname{card}(V \cap \mathcal{C})
$$

for each $j \geq 0$. Thus $\frac{1}{C_{j}} \mu_{V}^{0}(\mathcal{C})$ is at most $\frac{1}{C_{j}}$ card $(V \cap \mathcal{C})$, which tends to 0 as $j \rightarrow \infty$ by virtue of Lemma 4.9. Hence $\mu_{V}^{0}(\mathcal{C})=0$. 
4.11. Lemma. For any $V \in \mathfrak{V}$ and any point $z \in \mathbf{H}^{n}$, the sequence $\left(\frac{1}{C_{j}} \mu_{V, z, s_{j}}\right)$ converges weakly to $\mu_{V, z}$.

Proof. It is immediate from the definition that $d \mu_{V, z, s_{j}}=\bar{\Pi}_{\alpha}\left(z_{0}, z, \cdot\right)^{s_{j}} d \mu_{V, z_{0}, s_{j}}$, where $\bar{\Pi}_{\alpha}$ is the function given by Lemma 4.5. Setting $\mu_{j}^{0}=\frac{1}{C_{j}} \mu_{V, z_{0}, s_{j}}$ and $\mu_{j}=\frac{1}{C_{j}} \mu_{V, z, s_{j}}$, we therefore obtain, for any continuaous function $f$ on $\overline{\mathbf{H}}^{n}$ and any $j \geq 0$,

$$
\int f d \mu_{j}=\int \bar{\Pi}_{\alpha}\left(z_{0}, z, \cdot\right)^{s_{j}} f d \mu_{j}^{0}
$$

Now since $\lim _{j \rightarrow \infty} s_{j}=D$, and since $f$ and $\bar{\Pi}$ are continuous on the compact space $\overline{\mathbf{H}}^{n}$, we have $\bar{\Pi}_{\alpha}\left(z_{0}, z, \cdot\right)^{s_{j}} f \rightarrow \bar{\Pi}_{\alpha}\left(z_{0}, z, \cdot\right)^{D} f$ uniformly. Since the $\mu_{j}^{0}$ converge weakly to $\mu_{V}^{0}$, it follows that

$$
\lim _{j \rightarrow \infty} \int \bar{\Pi}_{\alpha}\left(z_{0}, z, \cdot\right)^{s_{j}} f d \mu_{j}^{0}=\int \bar{\Pi}_{\alpha}\left(z_{0}, z, \cdot\right)^{D} f d \mu_{V}^{0}=\int \mathcal{P}^{D} f d \mu_{V}^{0} .
$$

The right-hand side of the last equation is equal to $\int f d \mu_{V, z}$ since $\mathcal{M}_{V}=\left(\mu_{V, z}\right)$ is a $D$-conformal density. So we have

$$
\lim _{j \rightarrow \infty} \int f d \mu_{j}=\int f d \mu_{V, z}
$$

This proves the lemma.

Proof of Proposition 4.2.. In light of Lemma 4.10, for each $V \in \mathfrak{V}$, the measure $\mu_{V}^{0}$ may be regarded as a measure on $S_{\infty}$. According to 3.3, there exists for each $V \in \mathfrak{V}$ a unique $D$-conformal density $\mathcal{M}_{V}=\left(\mu_{V, z}\right)$ such that $\mu_{V, z_{0}}=\mu_{V}^{0}$. We shall prove Proposition 4.2 by showing that the family $\left(\mathcal{M}_{V}\right)$ of $D$-conformal densities satisfies conditions (i) - (iv) of the proposition.

To prove (i) it is enough to show that $\mu_{W, z_{0}} \neq 0$. But as we observed in 4.7 , for each $s>D$ and each $z \in \mathbf{H}^{n}$, the measure $\mu_{W, z, s}$ has total mass $\Sigma^{\prime}(z, s)$; in particular, for each $j \geq 0$ the total mass of $\mu_{W, z_{0}, s_{j}}$ is $C_{j}$. Thus $\mu_{W, z_{0}}$ is the weak limit of a sequence of measures of total mass 1 , and therefore itself has total mass 1 .

To prove (ii) we observe that if $V=\coprod_{i=1}^{m} V_{i}$, where $V$ and the $V_{i}$ belong to $\mathfrak{V}$, then for each $s>D$ and each $z \in \mathbf{H}^{n}$ we have $\mu_{V, z, s}=\sum_{i=1}^{m} \mu_{V_{i}, z, s}$ by the definitions of $\mu_{V, z, s}$ and $\mu_{V_{i}, z, s}$. Hence for each $j \geq 0$ we have

$$
\frac{1}{C_{j}} \mu_{V, z, s}=\frac{1}{C_{j}} \sum_{i=1}^{m} \mu_{V_{i}, z, s}
$$

Using Lemma 4.11 we can take weak limits of both sides to obtain

$$
\mu_{V, z}=\sum_{i=1}^{m} \mu_{V_{i}, z}
$$


This proves (ii).

To prove (iii) we must show that if $V$ is a set in $\mathfrak{V}$ and $\gamma$ is a hyperbolic isometry such that $\gamma V \in \mathfrak{V}$, then for each $z \in \mathbf{H}^{n}$ we have $\gamma_{\infty}^{*} \mu_{\gamma V, \gamma z}=\mu_{V, z}$. If we think of $\mu_{\gamma V, \gamma z}$ and $\mu_{V, z}$ as measures on $\overline{\mathbf{H}}^{n}$, then $\gamma_{\infty}^{*} \mu_{\gamma V, \gamma z}$ may be rewritten as $\bar{\gamma}^{*} \mu_{\gamma V, \gamma z}$. For each $s>D$ we have

$$
\bar{\gamma}^{*} \mu_{\gamma V, \gamma z, s}=\sum_{w \in V} e^{-s \alpha(\operatorname{dist}(\gamma w, \gamma z))} \bar{\gamma}^{*}\left(\delta_{\gamma w}\right)=\sum_{w \in V} e^{-s \alpha(\operatorname{dist}(w, \gamma z))} \delta_{w}=\mu_{V, z, s} .
$$

Hence for each $j \geq 0$ we have

$$
\bar{\gamma}^{*}\left(\frac{1}{C_{j}} \mu_{\gamma V, \gamma z, s_{j}}\right)=\frac{1}{C_{j}} \mu_{V, z, s_{j}} .
$$

Using Lemma 4.11 we can take weak limits of both sides to obtain $\bar{\gamma}^{*} \mu_{\gamma V, \gamma z}=\mu_{V, z}$, as required.

To prove (iv) we consider an arbitrary set $V \in \mathfrak{V}$. For any $s>D$, it follows from the definition of the measure $\mu_{V, z_{0}, s}$ that the support of $\mu_{V, z_{0}, s}$ is exactly $V \cup \Lambda_{V}$, the closure of $V$ in $\overline{\mathbf{H}}^{n}$. Hence supp $\frac{1}{C_{j}} \mu_{V, z_{0}, s_{j}}=V \cup \Lambda_{V}$ for each $j \geq 0$. By Lemma 4.11 it follows that $\operatorname{supp} \mu_{V}^{0} \subset V \cup \Lambda_{V}$. But $\operatorname{supp} \mu_{V}^{0} \subset S_{\infty}$ by 4.10, and hence $\operatorname{supp} \mu_{V}^{0} \subset \Lambda_{V}$. This implies (iv).

\section{§5. The Paradoxical DeCOMPOSITION}

This section is devoted to the proof of the following two results.

5.1. Proposition. Let $\Gamma$ be a Kleinian group which is free on a generating set $\Xi$ with $k$ elements, where $2 \leq k<\infty$. Suppose that every $\Gamma$-invariant conformal density for $\mathbf{H}^{3}$ is a constant multiple of the area density. Then for any $z \in \mathbf{H}^{3}$ we have

$$
\max _{\xi \in \Xi} \operatorname{dist}(z, \xi \cdot z) \geq \frac{1}{2} \log ((k-1)(2 k-1)) .
$$

5.2. Proposition. Let $\xi$ and $\eta$ be two orientation-preserving isometries of $\mathbf{H}^{3}$. Suppose that the group $\Gamma$ generated by $\xi$ and $\eta$ is discrete and is free on the generators $\xi$ and $\eta$. Suppose in addition that every $\Gamma$-invariant conformal density for $\mathbf{H}^{3}$ is a constant multiple of the area density. Then for any $z \in \mathbf{H}^{3}$ we have

$$
\max (\operatorname{dist}(z, \xi \cdot z), \operatorname{dist}(z, \eta \cdot z)) \geq \log 3
$$

5.3. The key step in the proofs of Propositions 5.1 and 5.2 is the following lemma. When the hypotheses of 5.1 or 5.2 are saøisfied, the lemma will give the "paradoxical" decomposition of the normalized area measure on $S_{\infty}$ which was described in the introduction. 
Lemma. Let $\Gamma$ be a Kleinian group which is free on a generating set $\Xi$. Set $\Psi=\Xi \amalg \Xi^{-1} \subset$ $\Gamma$. Let $z_{0}$ be any point of $\mathbf{H}^{3}$. Then there exist a number $D \in[0,2]$, a $\Gamma$-invariant $D$ conformal density $\mathcal{M}=\left(\mu_{z}\right)$ for $\mathbf{H}^{3}$ and a family $\left(\nu_{\psi}\right)_{\psi \in \Psi}$ of Borel measures on $S_{\infty}$ such that

(i) $\mu_{z_{0}}\left(S_{\infty}\right)=1$;

(ii) $\mu_{z_{0}}=\sum_{\psi \in \Psi} \nu_{\psi}$; and

(iii) for each $\psi \in \Psi$ we have

$$
\int\left(\lambda_{\psi, z_{0}}\right)^{D} d \nu_{\psi^{-1}}=1-\int d \nu_{\psi}
$$

If $\Xi$ is a two-element set $\{\xi, \eta\}$ and if $z_{0} \in \ell(\xi, \eta)$ (see 1.8) then we also have

(iv)

$$
\int d \nu_{\xi^{-1}}=\int d \nu_{\xi} \quad \text { and } \quad \int d \nu_{\eta^{-1}}=\int d \nu_{\eta}
$$

Proof. Every element $\gamma$ of $\Gamma$ can be written uniquely as a reduced word $\psi_{1} \cdots \psi_{m}$, where $m \geq 0$, each $\psi_{i}$ is an element of $\Psi$, and $\psi_{i+1} \neq \psi_{i}^{-1}$ for $i=1, \ldots, m-1$. If $\gamma \neq 1$, i.e. if $m>0$, we shall call $\psi_{1}$ the initial letter of $\gamma$. For each $\psi \in \Psi$, let $J_{\psi}$ denote the set of non-trivial elements of $\Gamma$ that have initial letter $\psi$. Then $\Gamma$ is a finite disjoint union

$$
\{1\} \amalg \coprod_{\psi \in \Psi} J_{\psi}
$$

Since $\Gamma$ is discrete and torsion-free, it acts freely on $\mathbf{H}^{3}$. Hence the orbit $W=\Gamma z_{0}$ is a disjoint union

$$
W=\left\{z_{0}\right\} \amalg \coprod_{\psi \in \Psi} V_{\psi}
$$

where $V_{\psi}=\left\{\gamma z: \gamma \in J_{\psi}\right\}$. Let $\mathfrak{V}$ denote the finite collection of all subsets of $W$ which are sub-unions of the above disjoint union; that is, $W$ consists of all sets of the form $\coprod_{\psi \in \Psi^{\prime}} V_{\psi}$ or $\left\{z_{0}\right\} \amalg \coprod_{\psi \in \Psi^{\prime}} V_{\psi}$ for $\Psi^{\prime} \subset \Psi$. We apply Proposition 4.2 with these choices of $W$ and $\mathfrak{V}$. We take $D$ to be a number, and $\left(\mathcal{M}_{V}\right)_{V \in \mathfrak{V}}$ a family of conformal densities, for which conditions (i)-(iv) of 4.2 are satisfied. We write $\mathcal{M}_{V}=\left(\mu_{V, z}\right)_{z \in \mathbf{H}^{n}}$. We set $\mathcal{M}=\mathcal{M}_{W}$, and $\nu_{\psi}=\mu_{V_{\psi}, z_{0}}$ for each $\psi \in \Psi$. According to $4.2, \mathcal{M}$ is $\Gamma$-invariant. It follows from condition (i) of 4.2 and the definition of a conformal density that $\mu_{z_{0}}=\mu_{W, z_{0}} \neq 0$; hence after multiplying by a constant we may assume that $\mu_{z_{0}}$ has total mass 1 . This is conclusion (i) of the present lemma. We shall show that the other conclusions of the lemma also hold with these definitions of $\mathcal{M}$ and of the $\nu_{\psi}$.

By (5.3.1) above and condition (ii) of 4.2, we have

$$
\mu_{z_{0}}=\mu_{W, z_{0}}=\mu_{\left\{z_{0}\right\}, z_{0}}+\sum_{\psi \in \Psi} \mu_{V_{\psi}, z_{0}} .
$$


But $\mu_{\left\{z_{0}\right\}, z_{0}}=0$ by condition (iv) of 4.2 . Hence

$$
\mu_{z_{0}}=\sum_{\psi \in \Psi} \mu_{V_{\psi}, z_{0}}=\sum_{\psi \in \Psi} \nu_{\psi}
$$

which is conclusion (ii) of the lemma.

It follows immediately from the definition of the sets $J_{\psi}$ that for each $\psi \in \Psi$ we have

$$
\psi J_{\psi^{-1}}=\Gamma-J_{\psi}
$$

so that

$$
\psi V_{\psi^{-1}}=W-V_{\psi}
$$

Since $W-V_{\psi}=\left\{z_{0}\right\} \amalg \coprod \psi^{\prime} \neq \psi V_{\psi^{\prime}} \in \mathfrak{V}$, condition (iii) of 4.2 gives

$$
\mathcal{M}_{V_{\psi}-1}=\psi_{\infty}^{*}\left(\mathcal{M}_{W-V_{\psi}}\right) \text {. }
$$

On the other hand, by $4.2(\mathrm{ii})$ we have

$$
\mathcal{M}=\mathcal{M}_{W-V_{\psi}}+\mathcal{M}_{V_{\psi}}
$$

Thus (5.3.2) can be rewritten in the form $\mathcal{M}_{V_{\psi^{-1}}}=\psi_{\infty}^{*}\left(\mathcal{M}-\mathcal{M}_{V_{\psi}}\right)$; and in particular we have

$$
\mu_{V_{\psi}-1, \psi\left(z_{0}\right)}=\psi_{\infty}^{*}\left(\mu_{z_{0}}-\mu_{V_{\psi}, z_{0}}\right)=\psi_{\infty}^{*}\left(\mu_{z_{0}}-\nu_{\psi}\right)
$$

Since $\mathcal{M}_{V_{\psi^{-1}}}$ is a $D$-conformal density, we have

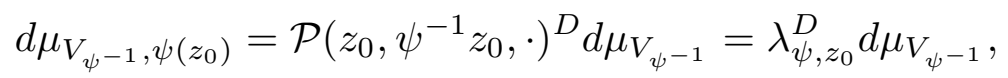

where the last equality follows from Proposition 2.4. Hence, equating the total masses of the two sides of (5.3.3), we obtain

$$
\int \lambda_{\psi, z_{0}}^{D} d \mu_{V_{\psi^{-1}}}=\int d\left(\psi_{\infty}^{*}\left(\mu_{z_{0}}-\nu_{\psi}\right)\right)=\int d\left(\mu_{z_{0}}-\nu_{\psi}\right)=1-\int d \nu_{\psi}
$$

and conclusion (iii) of the lemma is established.

It remains to show that conclusion (iv) holds if $\Xi=\{\xi, \eta\}$ and $z_{0} \in \ell(\xi, \eta)$. Since the involution $\tau=\tau_{\xi, \eta}$ satisfies $\tau \xi \tau=\xi^{-1}$ and $\tau \eta \tau=\eta^{-1}$, we have $\tau \Gamma \tau=\Gamma$; furthermore, $\tau J_{\xi} \tau=J_{\xi^{-1}}$ and $\tau J_{\eta} \tau=J_{\eta^{-1}}$. On the other hand, since $z_{0} \in \ell(\xi, \eta)$ we have $\tau z_{0}=z_{0}$. Hence $V_{\xi^{-1}}=\left\{\gamma z_{0}: \gamma \in J_{\xi^{-1}}\right\}=\left\{\tau \gamma \tau z_{0}: \gamma \in J_{\xi}\right\}=\tau V_{\xi}$. By condition (iii) of 4.2 it follows that $\tau_{\infty}^{*} \mathcal{M}_{V_{\xi}}=\mathcal{M}_{V_{\xi^{-1}}}$. In particular, $\tau_{\infty}^{*} \nu \xi=\nu_{\xi^{-1}}$. Taking total masses of both sides gives $\int d \nu_{\xi^{-1}}=\int d \nu_{\xi}$. The same argument shows that $\int d \nu_{\eta^{-1}}=\int d \nu_{\eta}$. This establishes (iv) and completes the proof of the lemma.

The proof of Propositions 5.1 and 5.2 will require combining Lemma 5.3.3 with the elementary considerations covered by the following two lemmas. 
5.4. Lemma. Let $\mu$ and $\mu_{0}$ be measures of finite total mass on a measurable space $(X, \mathcal{B})$. Suppose that $0 \leq \mu_{0} \leq \mu$. Let $C$ be a Borel set such that $\mu(C) \geq \mu_{0}(X)$. Let $f$ be a measurable, non-negative real-valued function on $X$ such that inf $f(C) \geq \sup f(X-C)$. Then

$$
\int_{X} f d \mu_{0} \leq \int_{C} f d \mu
$$

Proof. We write $\mu=\mu_{0}+\mu_{1}$, where $\mu_{1}$ is again a measure on $(X, \mathcal{B})$. We have

$$
\mu_{0}(X-C)=\mu_{0}(X)-\mu_{0}(C) \leq \mu(C)-\mu_{0}(C)=\mu_{1}(C),
$$

so that

$$
\int_{X-C} f d \mu_{0} \leq(\sup f(X-C)) \cdot \mu_{0}(X-C) \leq(\inf f(C)) \cdot \mu_{1}(C) \leq \int_{C} f d \mu_{1} .
$$

Hence

$$
\int_{X} f d \mu_{0}=\int_{C} f d \mu_{0}+\int_{X-C} f d \mu_{0} \leq \int_{C} f d \mu_{0}+\int_{C} f d \mu_{1}=\int_{C} f d \mu .
$$

5.5. Lemma. Let $a$ and $b$ be numbers such that $0 \leq a \leq \frac{1}{2}$ and $0 \leq b \leq 1$, let $\gamma$ be an isometry of $\mathbf{H}^{3}$ and let $z$ be a point in $\mathbf{H}^{3}$. Suppose that $\nu$ is a measure on $S_{\infty}$ such that

(i) $\nu \leq A_{z}$,

(ii) $\nu\left(S_{\infty}\right) \leq a$, and

Then

(iii) $\int_{S_{\infty}} \lambda_{\gamma, z}^{2} d \nu \geq b$.

$$
\operatorname{dist}(z, \gamma \cdot z) \geq \frac{1}{2} \log \frac{a(1-b)}{b(1-a)}
$$

Proof. We let $h$ denote the constant $\operatorname{dist}(z, \gamma \cdot z)$, and set $c=\cosh h$ and $s=\sinh h$. We let $\lambda$ denote the function $\lambda_{\gamma, z}$. We identify $\overline{\mathbf{H}}^{3}$ conformally with the unit ball in $\mathbf{R}^{3}$ in such a way that $z$ is the origin (so that $S_{\infty}$ has the round metric centered at $z$ ) and $\gamma^{-1} \cdot z$ is on the positive vertical axis.

According to 2.4, we have $\lambda(\zeta)=\mathcal{P}\left(z, \gamma^{-1} \cdot z, \zeta\right)$ for all $\zeta \in S_{\infty}$. Hence by (2.1.1), $\lambda$ is given by the formula

$$
\lambda(\zeta)=(c-s \cos \phi)^{-1}
$$

where $\phi=\phi(\zeta)$ is the angle between the positive vertical axis and the ray from the origin through $\zeta$; thus $\phi$ is the polar angle of $\zeta$ in spherical coordinates.

Set $A=A_{z}$. Since $S_{\infty}$ has the round metric centered at $z$, the measure $A$ is obtained by dividing the area measure on the unit sphere by the area $4 \pi$ of the sphere. In spherical coordinates $\theta$ and $\phi$ on the unit sphere we have $d A=(1 / 4 \pi) \sin \phi d \phi d \theta$. 
Set $\phi_{0}=\arccos (1-2 a)$, and let $C \subset S_{\infty}$ denote the spherical cap defined by the inequality $\phi \geq \phi_{0}$. Then we have

$$
A(C)=\frac{1}{4 \pi} \int_{0}^{2 \pi} \int_{0}^{\phi_{0}} \sin \phi d \phi d \theta=\frac{1}{2}\left(1-\cos \phi_{0}\right)=a .
$$

Thus by hypothesis (ii) we have $A(C) \geq \nu\left(S_{\infty}\right)$. Observe also that since $\lambda$ is given by the function $(c-s \cos \phi)^{-1}$, which is positive and monotone decreasing for $0 \leq \phi \leq \pi$, we have $\inf \lambda(C) \geq \sup \lambda(\Xi-C)$. Since we also have $\nu \leq A$ by hypothesis (i), we may apply Lemma 5.4 with $f=\lambda^{2}$ to obtain

$$
\begin{aligned}
\int_{S_{\infty}} \lambda^{2} d \nu & \leq \int_{C} \lambda^{2} d A=\frac{1}{4 \pi} \int_{0}^{2 \pi} \int_{0}^{\phi_{0}} \frac{\sin \phi}{(c-s \cos \phi)^{2}} d \phi d \theta \\
& =\frac{1}{2} \int_{0}^{\phi_{0}} \frac{\sin \phi}{(c-s \cos \phi)^{2}} d \phi=\frac{1}{2 s}\left(\frac{1}{c-s}-\frac{1}{c-\cos \phi_{0}}\right)
\end{aligned}
$$

where the last step follows from the substitution $u=c-s \cos \phi$. Recalling that $\cos \phi_{0}=$ $1-2 a$ and using hypothesis (iii), we find that

$$
b \leq \int_{S_{\infty}} \lambda^{2} d \nu \leq \frac{a}{(c-s)(c-s+2 a s)}
$$

After rewriting $c$ and $s$ respectively as $\frac{1}{2}\left(e^{h}+e^{-h}\right)$ and $\frac{1}{2}\left(e^{h}-e^{-h}\right)$, and simplifying, we conclude that

$$
e^{-2 h} \leq \frac{a(1-b)}{b(1-a)}
$$

Proof of Proposition 5.1. Suppose that $\Gamma$ and $\Xi$ satisfy the hypotheses of the proposition, and let $z_{0} \in \mathbf{H}^{n}$ be an arbitrary point.

We wish to show that

$$
\max _{\xi \in \Xi} \operatorname{dist}\left(z_{0}, \xi \cdot z_{0}\right) \geq \frac{1}{2} \log ((k-1)(2 k-1)) .
$$

In particular $\Gamma$ and $\Xi$ satisfy the satisfy the hypotheses of Lemma 5.3, and so there exist a number $D \in[0,2]$, a conformal density $\mathcal{M}=\left(\mu_{z}\right)$ and a family of Borel measures $\left(\nu_{\psi}\right)_{\psi \in \Psi}$ (where $\Psi=\Xi \amalg \Xi^{-1}$ ), for which conclusions (i)-(iii) of 5.3 hold. But the hypotheses of 5.1 imply that $\mathcal{M}$ is a constant multiple of the area density $\mathcal{A}$; hence $D=2$. Since $\mu_{z_{0}}$ has total mass 1 by $5.3(\mathrm{i})$, we must in fact have $\mathcal{M}=\mathcal{A}$.

By 5.3(ii) we have

$$
1=\sum_{\psi \in \Psi} \nu_{\psi}\left(S_{\infty}\right)=\sum_{\xi \in \Xi}\left(\nu_{\xi}\left(S_{\infty}\right)+\nu_{\xi^{-1}}\left(S_{\infty}\right)\right)
$$


Hence for some $\xi_{0} \in \Xi$ we have $\nu_{\xi_{0}}\left(S_{\infty}\right)+\nu_{\xi_{0}^{-1}}\left(S_{\infty}\right) \leq \frac{1}{k}$. It follows that for some $\psi_{0} \in\left\{\xi_{0}, \xi_{0}^{-1}\right\}$ we have $\nu_{\psi_{0}}\left(S_{\infty}\right) \leq \frac{1}{2 k}$.

We set $\lambda=\lambda_{\psi_{0}, z}$ and $\nu=\nu_{\psi_{0}}-1$. By 5.3(iii) we have

$$
\int_{S} \lambda^{2} d \nu=1-\nu_{\psi_{0}}\left(S_{\infty}\right) \geq 1-\left(\nu_{\xi_{0}}\left(S_{\infty}\right)+\nu_{\xi_{0}}-1\left(S_{\infty}\right)\right) \geq 1-\frac{1}{k} .
$$

Furthermore, it follows from 5.3(ii) that $\nu \leq \mu_{z_{0}}=A_{z_{0}}$. Hence we may apply Lemma 5.5 with $z=z_{0}, \gamma=\psi_{0}, a=\frac{1}{2 k}$ and $b=1-\frac{1}{k}$. This gives

$$
\operatorname{dist}\left(z, \psi_{0} \cdot z\right) \geq \frac{1}{2} \log ((k-1)(2 k-1)) .
$$

But since $\psi_{0}=\xi_{0}^{ \pm 1}$ we have $\operatorname{dist}\left(z, \psi_{0} \cdot z\right)=\operatorname{dist}\left(z, \xi_{0} \cdot z\right)$, and the conclusion follows.

Proof of Proposition 5.2. Suppose that $\Gamma, \xi$ and $\eta$ satisfy the hypotheses of the proposition. Let $z_{0} \in \mathbf{H}^{n}$ be any point; we wish to show that $\max \left(\operatorname{dist}\left(z_{0}, \xi \cdot z_{0}\right), \operatorname{dist}\left(z_{0}, \eta \cdot z_{0}\right)\right) \geq \log 3$. Set $\ell=\ell(\xi, \eta)$. It follows from Proposition 1.15 that

$$
\max \left(\operatorname{dist}\left(z_{0}, \xi \cdot z_{0}\right), \operatorname{dist}\left(z_{0}, \eta \cdot z_{0}\right)\right) \geq \max \left(\operatorname{dist}\left(z_{1}, \xi \cdot z_{1}\right), \operatorname{dist}\left(z_{1}, \eta \cdot z_{1}\right)\right),
$$

where $z_{1}$ denotes the orthogonal projection of $z_{0}$ onto $\ell$. Hence we may assume without loss of generality that $z_{0} \in \ell$.

In particular $\Gamma$ and $\Xi=\{\xi, \eta\}$ satisfy the hypotheses of Lemma 5.3, and so there exist a number $D \in[0,2]$, a conformal density $\mathcal{M}=\left(\mu_{z}\right)$ and a family of Borel measures $\left(\nu_{\psi}\right)_{\psi \in \Psi}$ (where $\Psi=\left\{\xi, \xi^{-1}, \eta, \eta^{-1}\right\}$ ), for which conclusions (i)-(iv) of 5.3 hold. But the hypotheses of 5.2 imply that $\mathcal{M}$ is a constant multiple of the area density $\mathcal{A}$; hence $D=2$. Since $\mu_{z_{0}}$ has total mass 1 by $5.3(\mathrm{i})$, we must in fact have $\mathcal{M}=\mathcal{A}$.

By conclusions (ii) and (iv) of 5.3 we have

$$
1=2 \nu_{\xi}\left(S_{\infty}\right)+2 \nu_{\eta}\left(S_{\infty}\right)
$$

so that either $\nu_{\xi}\left(S_{\infty}\right)$ or $\nu_{\eta}\left(S_{\infty}\right)$ is $\leq 1 / 4$. By symmetry we may assume that $\nu_{\xi}\left(S_{\infty}\right) \leq 1 / 4$. We set $\lambda=\lambda_{\xi^{-1}, z_{0}}$. By 5.3(iii) and (iv) we have

$$
\int_{S} \lambda^{2} d \nu_{\xi}=1-\nu_{\xi^{-1}}\left(S_{\infty}\right)=1-\nu_{\xi}\left(S_{\infty}\right) \geq 3 / 4
$$

Furthermore, it follows from 5.3(i) that $\nu_{\xi} \leq A$. Hence we may apply Lemma 5.5 with $\nu=\nu_{\xi}, \gamma=\psi_{0}, a=\frac{1}{4}$ and $b=\frac{3}{4}$. This gives

$$
\operatorname{dist}\left(z_{0}, \xi^{-1} \cdot z_{0}\right) \geq \log 3 .
$$

Since $\operatorname{dist}\left(z_{0}, \xi \cdot z_{0}\right)=\operatorname{dist}\left(z_{0}, \xi^{-1} \cdot z_{0}\right)$, the conclusion follows. 


\section{$\S 6$. BONAHON SURFACES AND SUPERHARMONIC FUNCTIONS}

The results of Section 5 depend on the hypothesis that every $\Gamma$-invariant conformal density is a constant multiple of the area density, where $\Gamma$ is the given free Kleinian group. According to Proposition 3.9, this is always true if every positive, $\Gamma$-invariant superharmonic function on $\mathbf{H}^{3}$ is constant. Thus in order to apply the results of Section 5 one needs a geometric condition that guarantees that the hyperbolic manifold $M(\Gamma)$ admits no non-constant positive superharmonic functions. In this section we describe such a condition. Very similar conditions have been used in Thurston [Th 8.12] and, later, in Sullivan [Su3], Bohanon [Bo] and Canary [Ca1].

For our applications we need only consider Kleinian groups $\Gamma$ which contain no parabolics and satisfy $M(\Gamma)=N(\Gamma)$. We will restrict attention to these groups whenever convenient. While the complications which arise from the existence of parabolics can be dealt with, it would unnecessarily complicate the exposition to do so here.

6.1. Let $\Sigma$ be a closed 2 -manifold, not necessarily connected. Let $u \in H_{2}(\Sigma ; \mathbf{Z} / 2)$ denote the fundamental class of $\Sigma$, i.e. the sum of the images under inclusion of the fundamental classes of the components of $\Sigma$. A map $f: \Sigma \rightarrow M$ will be said to be null-homologous if the class $f_{*}(u) \in H_{2}(M ; \mathbf{Z} / 2)$ is trivial. Suppose that $f: \Sigma \rightarrow M$ is a null-homologous map and that $P$ is a point of $M-f(P)$. We will say that $P$ is strictly enclosed by $f$ if the class $f_{*}(u) \in H_{2}(M-\{P\} ; \mathbf{Z} / 2)$ is non-trivial, where we are regarding $f$ as a map from $\Sigma$ to $M-\{P\}$. We will say that a point $P \in M$ is enclosed by the null-homologous map $f$ if either $P \in f(\Sigma)$ or $P$ is strictly enclosed by $f$. A compact set $K$ will be said to be enclosed by $f$ if every point of $K$ is enclosed by $f$.

Proposition. Let $\Gamma$ be a Kleinian group without parabolics such that $M(\Gamma)=N(\Gamma)$. Suppose that $f_{j}: \Sigma_{j} \rightarrow M$ is a sequence of maps from closed 2-manifolds into $M$ such that every compact subset of $M(\Gamma)$ is enclosed by some $f_{j}$. If there exists a constant $V$ such that the volume of $\operatorname{nbhd}_{1} f_{j}\left(\Sigma_{j}\right)$ is less than $V$ for all $j$ then every positive superharmonic function on $M$ is constant.

Proof. This statement is proved by an argument given in [Th, 8.12] and [Ca, Theorem 7.1]. In that argument it is assumed that $M$ has $k$ simply degenerate ends with neighborhoods homeomorphic to $S_{1} \times[0, \infty), \ldots, S_{k} \times[0, \infty)$. The argument is applied to a sequence of maps $f_{j}: \Sigma \rightarrow M$ where $\Sigma=\Sigma_{j}$ is the disjoint union of $S_{1}, \ldots, S_{k}$ and where the restriction of $f_{j}$ to $S_{i}$ is homotopic to the inclusion of $S_{j} \times\{0\}$ into $M$. The argument uses two properties of these maps. First that there is a bound on the volume of the sets $\operatorname{nbhd}_{1}\left(f_{j}\left(\Sigma_{j}\right)\right)$ and second that a flow line which exits an end of $M$ must pass through infinitely many of these sets. The second property follows immediately from our hypothesis that every compact set in $M$ is enclosed by one of the maps $f_{j}$.

The rest of this section is devoted to a description of a geometric construction, due to Bonahon, of maps that satisfy the hypotheses of Proposition 6.1. The construction is a simplification of Thurston's construction of "pleated surfaces."

6.2. Virtual triangulations. A mapping $\varphi$ of an affine $k$-simplex $\Delta^{k}$ into a topological space $X$ will be said to define a virtual k-simplex in $X$ if $\varphi$ is one-to-one on the interior of each face of $\Delta^{k}$. Two maps $\varphi: \Delta \rightarrow X$ and $\varphi^{\prime}: \Delta^{\prime} \rightarrow X$ will be said to define the same 
virtual simplex if there is an affine isomorphism $I: \Delta \rightarrow \Delta^{\prime}$ such that $\varphi \circ I=\psi$. By the interior of the virtual simplex (defined by) $\varphi: \Delta \rightarrow X$ we mean the set $\varphi($ int $\Delta$ ). The dimension of $\varphi$ is the dimension of $\Delta$. A virtual simplex $\varphi_{1}: \Delta_{1} \rightarrow X$ is called a face of a virtual simplex $\varphi$ if there is an affine isomorphism $J$ of $\Delta_{1}$ onto a face of $\Delta$ such that $\varphi \circ J=\varphi_{1}$.

Let $\Sigma$ be a topological 2-manifold-with-boundary. (We may have $\partial \Sigma=\emptyset$, and $\Sigma$ may be disconnected.) A virtual triangulation of $\Sigma$ is a collection $\Phi$ of virtual simplices in $\Sigma$ such that

(i) $\Phi$ contains the faces of each simplex in $\Phi$, and

(ii) as a set, $\Sigma$ is the disjoint union of the interiors of the virtual simplices in $\Phi$. (Note that two faces of a simplex may coincide.)

For any integer $i$, the $i$-skeleton of $\Sigma$ in a given virtual triangulation is defined to be the union of the interiors of all the virtual simplices of dimension $\leq i$; we denote it by $\operatorname{skel}_{i}(K)$. The virtual 0 -simplices of $\Sigma$ will be called vertices.

A virtual 1-simplex $\varphi$ in the 2-manifold-with-boundary $\Sigma$ may be regarded as a (nonoriented) path in $\Sigma$. If $\varphi$ is a homotopically trivial loop we shall say that it is degenerate. A virtual triangulation will be termed non-degenerate if it has no degenerate 1-simplices.

6.3. Piecewise hyperbolic structures. Let $\varphi: \Delta \rightarrow \Sigma$ be a virtual 2-simplex in a topological surface $\Sigma$. A homeomorphism $h$ of $\Delta=$ domain $\varphi$ onto a triangle in $\mathbf{H}^{2}$ which maps vertices onto vertices will be said to define a hyperbolic structure on $\varphi$. Two such homeomorphisms $h$ and $h^{\prime}$ will be said to define the same hyperbolic structure on $\varphi$ if $h^{\prime} h^{-1}$ is an isometry of hyperbolic triangles. If $\psi: \Delta_{1} \rightarrow \Sigma$ is a 1-dimensional face of $\varphi$, any hyperbolic structure on $\varphi$ induces a linear metric on $\Delta_{1}$.

A virtual 1-simplex in the interior of a virtually triangulated surface $\Sigma$ either occurs as a face of two distinct 2 -simplices or as two coincident faces of a single 2 -simplex. Thus if each 2-simplex in $\Sigma$ is given a hyperbolic structure then for each interior 1-simplex there are two linear metrics induced on $\Delta_{1}$. A piecewise-hyperbolic $(\mathrm{PH})$ structure on $\Sigma$ is a family of hyperbolic structures on the virtual 2-simplices of $\Sigma$ such that, for every interior 1-simplex of $\Sigma$, the two induced linear metrics on $\Delta_{1}$ agree. A PH-structure on $\Sigma$ determines, in a natural way, the structure of a (necessarily incomplete) hyperbolic 2-manifold with geodesic boundary on $\Sigma-\operatorname{skel}_{0}(\Sigma)$,

If $\varphi: \Delta \rightarrow \Sigma$ is a virtual 2-simplex and $w$ is a vertex of $\Delta$, we shall denote by $\theta_{w}(\varphi)$ the angle in the hyperbolic triangle $\Delta$ at the vertex $w$. For any vertex $x$ of $\Sigma$ we define the angle sum at $x$ in a given triangulation to be $\sum_{\varphi, w} \theta_{w}(\varphi)$, where $\varphi$ ranges over all virtual 2 -simplices of the given virtual triangulation, and $w$ ranges over all the vertices of $\operatorname{dom} \varphi$ which are mapped by $\varphi$ to $x$. (Note that a given virtual 2 -simplex $\varphi$ can contribute as many as three terms to this sum.) If $x$ is a point of $\Sigma$ which is not a vertex, we define the angle sum at $x$ to be $2 \pi$ if $x \in$ int $\Sigma$ and $\pi$ if $x \in \partial \Sigma$.

Let $x$ be any point of $\Sigma$ and let $a$ denote the angle sum at $x$. If $x \in$ int $\Sigma$ we set $\operatorname{excess}(x)=a-2 \pi$. If $x \in \partial \Sigma$ we set $\operatorname{excess}(x)=a-\pi$. We call $x$ a singular point if $\operatorname{excess}(x) \neq 0$. If $x \in \partial \Sigma$ and excess $(x)<0$, we call $x$ a corner. We shall denote by $\operatorname{sing} \Sigma$ the set of all singular points of $\Sigma$. It is clear that the natural hyperbolic structure on $\Sigma-\operatorname{skel}_{0}(\Sigma)$ admits a unique extension to a hyperbolic structure on $\Sigma-\operatorname{sing} \Sigma$.

Given a virtual 2-simplex $\varphi: \Delta \rightarrow \Sigma$ in the PH surface $\Sigma$, a path in $\varphi(\Delta)$ will be called 
geodesic if it has the form $\varphi \circ \alpha$ for some geodesic path $\alpha$ (parametrized proportionally to arc length) in $\Delta$. A path in $\Sigma$ will be termed piecewise geodesic (PG) if it is a composition $\gamma=\left(\varphi_{1} \circ \alpha_{1}\right) * \cdots *\left(\varphi_{n} \circ \alpha_{n}\right)$ of finitely many geodesic paths supported in 2-simplices of $\Sigma$. The length of $\gamma$ is the sum of the lengths of the $\alpha_{i}$. Any two points in the same component of $\Sigma$ can be joined by a PG path, and it is not hard to show that a metric on each component of $\Sigma$ can be constructed by defining $\operatorname{dist}(x, y)$ to be the infimum of the lengths of PG paths joining $x$ and $y$. It is also not difficult to verify that this metric agrees with the hyperbolic metric on the components of $\Sigma-\operatorname{sing} \Sigma$.

6.4. Ultrahyperbolic surfaces. We define an ultrahyperbolic structure on a virtually triangulated surface $\Sigma$ to be a PH structure such that excess $(x) \geq 0$ for every $x \in \operatorname{int} \Sigma$. The ultrahyperbolic surfaces form a rich class of geometric objects which are natural generalizations of hyperbolic surfaces. For example, by a generalization of the Gauss-Bonnet theorem which applies to $\mathrm{PH}$ surfaces (c.f. [Bo, Lemme 1.9]), each component of a surface with an ultrahyperbolic structure must have negative Euler characteristic. The fundamental geometric property of these surfaces which we will need here is the version of Bonahon's Bounded Diameter Lemma stated below.

Given an ultrahyperbolic surface $\Sigma$ and a point $P \in \Sigma$ we define $\operatorname{short}(P)$ to be the infinum of the lengths of homotopically non-trivial PG loops based at $P$. If $I$ is an interval contained in $(0, \infty)$ we define $\Sigma_{I}$ as in 1.5. In particular, for $\epsilon>0$, the $\epsilon$-thin part of $\Sigma$ is the subset $\Sigma_{(0, \epsilon]}$. Given a PG path $\gamma$ in $\Sigma$ we define the length of $\gamma$ modulo $\Sigma_{(0, \epsilon]}$ to be the length, i.e. the Lebesgue measure, of $\gamma^{-1}\left(\Sigma_{(\epsilon, \infty)}\right)$. If $P$ and $Q$ are points of $\Sigma$ then the distance from $P$ to $Q$ modulo $\Sigma_{(0, \epsilon]}$ is the infimum of the set of lengths modulo $\Sigma_{(0, \epsilon]}$ of PG paths from $P$ to $Q$. The diameter of $\Sigma$ modulo $\Sigma_{(0, \epsilon]}$ is the supremum over all $P, Q \in \Sigma$ of the distance modulo $\Sigma_{(0, \epsilon]}$ from $P$ to $Q$.

The following result is due to Bonahon [Bo, Lemme 1.10].

Proposition. For each positive integer $g$ and each positive number $\epsilon$ there exists a constant $D(g, \epsilon)$ such that if $\Sigma$ is a closed ultrahyperbolic surface of total genus $g$ then the diameter of $\sigma$ modulo $\Sigma_{(0, \epsilon]}$ is less than $D(g, \epsilon)$.

6.5. Hyperbolically simplicial surfaces. Let us consider a complete hyperbolic manifold $M=\mathbf{H}^{n} / \Gamma$. A map $f$ of a virtually triangulated (not necessarily connected) surface $\Sigma$ into $M$ will be called hyperbolically simplicial if for every virtual simplex $\varphi: \Delta \rightarrow \Sigma$, the map $f_{\varphi}=f \circ \varphi$ admits a continuous lift $\widetilde{f}_{\varphi}: \Delta \rightarrow \mathbf{H}^{n}$ such that $\widetilde{f}_{\varphi}(\Delta)$ is a hyperbolic simplex and either (i) $\operatorname{dim} \widetilde{f}_{\varphi}(\Delta)<\operatorname{dim} \Delta$ or (ii) $\tilde{f}_{\varphi}$ maps $\Delta$ homeomorphically onto $\tilde{f}_{\varphi}(\Delta)$. If (ii) holds for every simplex of $\Sigma$ we shall say that $f$ is non-degenerate. If $f$ is non-degenerate then for each virtual 2 -simplex $\varphi$ the map $\widetilde{f}_{\varphi}$ induces a hyperbolic structure on the 2 -simplex $\Delta$ which is obviously independent of the choice of lift. The linear metric induced on the domain of a 1-simplex $\psi$ by an adjacent 2 -simplex agrees with the linear metric determined by $\widetilde{f}_{\psi}$. Thus a non-degenerate hyperbolically simplicial map from $\Sigma$ to $M$ induces a $\mathrm{PH}$ structure on $\Sigma$. If we equip $\Sigma$ with the $\mathrm{PH}$ structure induced by the non-degenerate hyperbolically simplicial map $f$, then for every $\mathrm{PG}$ path $\gamma$ in $\Sigma$, the path $f \circ \gamma$ has the same length as $\gamma$. Hence if we give each component of $\Sigma$ the metric defined by its $\mathrm{PH}$ structure, the restriction of $f$ to each component of $\Sigma$ is distance-decreasing. 
By a hyperbolically simplicial surface in $M$ we mean a pair $(\Sigma, f)$, where $\Sigma$ is a closed, orientable surface equipped with a virtual triangulation, and $f: \Sigma \rightarrow M$ is a hyperbolically simplicial map. We shall say that $(\Sigma, f)$ is non-degenerate if the virtual triangulation of $\Sigma$ and the map $f$ are non-degenerate. The Euler characteristic and the total genus of a hyperbolically simplicial surface $(\Sigma, f)$ are defined respectively to be the Euler characteristic and the total genus of the surface $\Sigma$.

Suppose we are given a hyperbolically simplicial surface $(\Sigma, f)$. Then any small perturbation of $f \mid \operatorname{skel}_{0} \Sigma$ can be extended over $\Sigma$ to give a hyperbolically simplicial surface $\left(\Sigma, f^{\prime}\right)$ such that $f^{\prime}$ is homotopic to $f$. One first modifies $f$ in a neighborhood of the 0 -skeleton to make it agree with $f^{\prime}$ on the vertices. The restrictions to $\operatorname{skel}_{1} \Sigma$ and $\operatorname{skel}_{2} \Sigma$ can then be successively modified to make them geodesic. The map $f^{\prime}$ can be made arbitrarily close to $f$ in the uniform topology by choosing the initial perturbation to be sufficiently small.

We will say that a hyperbolically simplicial surface $(\Sigma, f)$ encloses a compact set $K \subset M$ provided that the map $f: \Sigma \rightarrow M$ is null-homologous and encloses $K$.

6.6. Bohanon surfaces. A continuous map $f$ of a topological 1-manifold $S$ into a hyperbolic 3-manifold $M$ will be called geodesic if for every component $C$ of $S$ there is a covering map $p: \mathbf{R} \rightarrow C$ such that $f \circ p$ is a geodesic. In the case where $S$ is a simple closed curve, i.e. is homeomorphic to $S^{1}$, a geodesic map $f: S \rightarrow M$ is a re-parametrization of a closed geodesic in $M$, and thus has a well-defined length.

A non-degenerate hyperbolically simplicial surface $(\Sigma, f)$ in $M$ will be called a Bonahon surface if there is a closed 1-manifold $S \subset \Sigma$ such that $\operatorname{skel}_{0}(\Sigma) \subset S \subset \operatorname{skel}_{1}(\Sigma)$ and $f \mid S$ is geodesic. The following observation is contained in [Bo, Lemme 1.8].

Proposition. If $(\Sigma, f)$ is a Bonahon surface in a complete hyperbolic manifold $M$, then the PH structure induced on $\Sigma$ by $f$ is ultrahyperbolic.

Proof. We must show that the angle sum at any vertex $x$ of $\Sigma$ is at least $2 \pi$. Let $e_{1}, \ldots, e_{n}$ be the distinct oriented virtual 1-simplices of $\Sigma$ having $x$ as initial point. Then $f$ maps each $e_{i}$ to a geodesic path $\gamma_{i}:[0,1] \rightarrow \Sigma$ with initial point $x$. Let $U$ denote the unit sphere in the tangent space to the three manifold $M$ at $f(x)$. Let $u_{i} \in U$ be the normalized tangent vector to $\gamma_{i}$ at 0 . Then the angle sum at $x$ is equal to the length of a piecewise-geodesic curve $\gamma$ in $U$ whose vertices are $u_{1}, \ldots, u_{n}$. Now according to the definition of a Bonahon surface, the vertex $x$ lies on a simple closed curve $C \subset \operatorname{skel}_{1}(\Sigma)$ such that $f \mid C$ is geodesic. This implies that the curve $\gamma$ passes through two antipodal points of $U$, and therefore has length $\geq 2 \pi$.

6.7. Neighborhoods of Bonahon surfaces. Let $(\Sigma, f)$ be a Bonahon surface in $M$, and let $\Sigma$ be given the PH structure induced by $f$. Let $\epsilon$ be a positive number. We shall say that $(\Sigma, f)$ is $\epsilon$-incompressible if for every homotopically non-trivial PG closed curve $\gamma: S^{1} \rightarrow \Sigma$ of length $<\epsilon$, the closed curve $f \circ \gamma$ is homotopically non-trivial in $M$.

The following proposition is proved in [Ca, Lemma 7.2]. (The pleated surface version is found in [Th, 8.12.1].)

Proposition. For each positive integer $g$ and each positive number $\epsilon$ there exists a constant $V(g, \epsilon)$ such that if $(f, \Sigma)$ is any $\epsilon$-incompressible Bonahon surface of genus $g$ in a complete hyperbolic 3-manifold $M$ then the volume of $\operatorname{nbhd}_{1}(f(\Sigma))$ is at most $V(g, \epsilon)$. 
6.8. The following Proposition, which is an immediate consequence of Propositions 6.1 and 6.7, gives a basic criterion for every positive superharmonic function on $M(\Gamma)$ to be constant.

Proposition. Let $\Gamma$ be a Kleinian group without parabolics such that $M(\Gamma)=N(\Gamma)$. Suppose that there exist positive integers $g_{1}, \ldots, g_{k}$ and a real number $\epsilon$ such that every compact subset of $M(\Gamma)$ is enclosed by a system $\left(\Sigma_{1}, f_{1}\right), \ldots,\left(\Sigma_{k}, f_{k}\right)$ of $\epsilon$-incompressible Bonahon surfaces such that the genus of $\Sigma_{i}$ is less than $g_{i}$. Then every positive superharmonic function on $M(\Gamma)$ is constant.

6.9. Topological tameness. A 3-manifold is said to be topologically tame if it is homeomorphic to the interior of a compact 3-manifold. A Kleinian group $\Gamma$ is topologically tame if $M(\Gamma)$ is topologically tame.

A Theorem due to R. Canary [Ca1] implies that if $\Gamma$ is a Kleinian group without parabolics such that $M(\Gamma)=N(\Gamma)$ and if $\Gamma$ is topologically tame then the hypotheses of Proposition 6.8 hold. In particular we have the following special case of [Ca, Theorem $7.2]$.

Proposition. Let $\Gamma$ be a Kleinian group without parabolics such that $M(\Gamma)=N(\Gamma)$. If $\Gamma$ is topologically tame then every positive super-harmonic function on $M(\Gamma)$ is constant.

In Section 8 we show, by quite different methods, that the hypotheses of Proposition 6.8 hold for most rank-2 free Kleinian groups which have no parabolics and lie on the boundary of the space of rank-2 free geometrically finite groups. The proof of our main theorem in Section 9 breaks into two cases which are handled respectively by the results of Section 8 and the proposition above.

6.10. We conclude this section with a result that will be needed in Section 8 .

Proposition. Let $M$ be a 3-manifold with an involution $T$ such that every connected component of Fix $T$ is homeomorphic to $\mathbf{R}$. Let $(\Sigma, f)$ be a null-homologous hyperbolically simplicial surface in $M$, and let $T_{\Sigma}$ be an orientation-preserving involution of $\Sigma$ such that $f \circ T_{\Sigma}=T \circ f$. Suppose that for some component $L$ of Fix $T$ there are exactly two fixed points $v_{+}$and $v_{-}$of $T_{\Sigma}$ which are mapped into $L$ by $f$; let $s$ denote the compact arc in $L$ bounded by $f\left(v_{+}\right)$and $f\left(v_{-}\right)$. Then $(\Sigma, f)$ encloses $s$.

Proof. It is enough to prove that every point of $s \cap(M-f(\Sigma))$ is strictly enclosed by $(\Sigma, f)$. Let $P$ be any point of $s \cap(M-f(\Sigma))$. By a small perturbation, as described in 6.5, one can approximate the map $f$ arbitrarily well, in the uniform topology on the space of maps of $\Sigma$ into $M$, by a hyperbolically simplicial map $f_{1}$ which is topologically transverse to $L$ and satisfies $f_{1} \circ T_{\Sigma}=T \circ f_{1}$. In particular, $f_{1}$ maps the finite set Fix $T_{\Sigma}$ into the 1-manifold Fix $T$. Hence if $f_{1}$ is close enough to $f$ then $f_{1}$ maps $v_{+}$and $v_{-}$into distinct components of $L-\{P\}$ and maps the remaining fixed points of $T_{\Sigma}$ into (Fix $\left.T\right)-L$. Thus the hypotheses of the theorem hold when $f$ is replaced by $f_{1}$. Furthermore, if $f_{1}$ is close enough to $f$ then $f_{1}$ maps $\Sigma$ into $M-\{P\}$ and is homotopic in $M-\{P\}$ to $f$. Hence $f_{1}$ strictly encloses $P$ if and only if $f$ does. Thus we may assume without loss of generality that $f$ is topologically transverse to $L$.

In this case the set $f^{-1}(L)$ is finite, and has even cardinality since $(\Sigma, f)$ is nullhomologous. Let $L_{+}$and $L_{-}$denote the components of $L-\{P\}$ containing $f\left(v_{+}\right)$and 
$f\left(v_{-}\right)$respectively. To say that $(\Sigma, f)$ encloses $P$ is equivalent to saying that $f^{-1}\left(L_{+}\right)$(and hence also $f^{-1}\left(L_{-}\right)$) has odd cardinality. But $f^{-1}\left(L_{+}\right)$is invariant under the involution $T_{\Sigma}$ (since $f \circ T_{\Sigma}=T \circ f$ ) and contains only one fixed point of $T_{\Sigma}$, namely $a$. Hence card $f^{-1}\left(L_{+}\right)$is indeed odd.

\section{$\S 7$. Girded surfaces AND GIRDED 3-MANifoldS}

In this section we introduce a special class of Bonahon surfaces which will be used in the proof of our main theorem.

7.1. Let $\epsilon$ be a positive number. By an $\epsilon$-girded surface in $M$ we mean a triple $(\Sigma, f, S)$, where $(\Sigma, f)$ is a non-degenerate hyperbolically simplicial surface in $M$ and $S \subset \Sigma$ is a closed 1-manifold, such that

(i) $\operatorname{skel}_{0}(\Sigma) \subset S \subset \operatorname{skel}_{1}(\Sigma)$;

(ii) each component of $\Sigma-S$ is homeomorphic to a sphere with three punctures;

(iii) $f \mid S$ is geodesic, and $f \mid C$ has length $<\epsilon / 3$ for each component $C$ of $S$; and

(iv) for each component $R$ of $\Sigma-S$, the map $f \mid R$ induces an injective homomorphism from $\pi_{1}(R)$ to $\pi_{1}(M)$.

The maps $f \mid C$, where $C$ ranges over the components of $S$, are re-parametrizations of closed geodesics. These closed geodesics will be said to be carried by $(\Sigma, f, S)$. It follows from condition (ii) above that $S$ has $3 g-3$ components, where $g$ is the genus of $\Sigma$. Hence if $k$ denotes the number of distinct closed geodesics carried by $\Sigma$, we have $1 \leq k \leq 3 g-3$. It follows from the definitions that if $(\Sigma, f, S)$ is an $\epsilon$-girded surface then $(\Sigma, f)$ is a Bonahon surface. Note also that since no component of $\Sigma$ is a sphere, condition (iv) of the above definition implies that the closed geodesics carried by $(\Sigma, f, S)$ are non-trivial; in particular the components of $S$ are homotopically non-trivial in $\Sigma$.

7.2. Proposition. Let $\epsilon$ be a Margulis number for $M$, and let $(\Sigma, f, S)$ be an $\epsilon / 3$-girded surface in $M$. Then $(\Sigma, f, S)$ is $\epsilon / 3$-incompressible.

Proof. Let $\gamma: S^{1} \rightarrow \Sigma$ be any homotopically non-trivial PG closed curve of length $<\epsilon / 3$. If $\gamma\left(S^{1}\right)$ is disjoint from $S$, then by 7.1(iv), $f \circ \gamma$ is homotopically non-trivial in $M$.

Now suppose that $\gamma\left(S^{1}\right)$ meets a component $C$ of $S$. Then $f\left(\gamma\left(S^{1}\right)\right)$ meets the set $f(C)$, which by 7.1(iii) is the support of a closed geodesic of length $\epsilon / 3$ in $M$. In particular $f\left(\gamma\left(S^{1}\right)\right)$ meets $M_{(0, \epsilon / 3]}$. But since $\gamma$ has length $<\epsilon / 3$ and $f$ is distance-decreasing, $f\left(\gamma\left(S^{1}\right)\right)$ has diameter $<\epsilon / 3$ and hence $f\left(\gamma\left(S^{1}\right)\right) \subset \operatorname{nbhd}_{\epsilon / 3}\left(M_{(0, \epsilon / 3]}\right) \subset M_{(0, \epsilon)}$. Since $\epsilon$ is a Margulis number for $M$, the component $\mathcal{T}$ of $M_{(0, \epsilon)}$ containing $f\left(\gamma\left(S^{1}\right)\right)$ is a Margulis tube, and the core of $\mathcal{T}$ is the only closed geodesic meeting $\mathcal{T}$. Hence if $S^{\prime} \supset C$ denotes the union of all components of $S$ that meet $\gamma\left(S^{1}\right)$, then $f\left(S^{\prime} \cup \gamma\left(S^{1}\right)\right) \subset \mathcal{T}$.

Let $Z$ denote the connected set $S^{\prime} \cup \gamma\left(S^{1}\right)$. Since $Z$ is a finite union of geodesic arcs, it is a subcomplex of $\Sigma$ in some triangulation. Hence there is a connected neighborhood $U$ of $Z$ in $\Sigma$ such that $Z$ carries $\pi_{1}(U)$. We may take $U$ to be a compact 2-manifold-with-boundary contained in $f^{-1}(\mathcal{T})$. Now we let $U^{\prime}$ denote the 2-manifold-with-boundary obtained by adjoining to $U$ all disk components of $\Sigma-U$, and we let $U^{\prime \prime}$ denote the complement in $U^{\prime}$ of a regular neighborhood of $S^{\prime}$ in int $U^{\prime}$. Then $\pi_{1}\left(U^{\prime}\right)$ is carried by $Z \subset f^{-1}(\mathcal{T})$, and hence $\left(f \mid U^{\prime}\right)_{\#}\left(\pi_{1}\left(U^{\prime}\right)\right) \subset \pi_{1}(M)$ is cyclic. On the other hand, it follows from 7.1(iv) and 
the construction of $U^{\prime \prime}$ that $f$ induces an injection from the fundamental group of each component of $U^{\prime \prime}$ into $\pi_{1}(M)$. Hence each component of $U^{\prime \prime}$ has cyclic fundamental group and must therefore be an annulus.

This implies that $U^{\prime}$ has Euler characteristic zero. Since $\Sigma$ has genus $>1, U^{\prime}$ must be an annulus. Since $C$ is a homotopically non-trivial simple closed curve in $U^{\prime}$, it is a topological core of $U^{\prime}$. But $\gamma$ is a homotopically non-trivial closed curve in $U^{\prime}$ and is therefore homotopic in $U^{\prime}$ to a power of $C$. As $f \mid C$ is a non-trivial closed geodesic, and as the complete hyperbolic manifold $M$ necessarily has torsion-free fundamental group, we conclude that $f \circ \gamma$ is homotopically non-trivial as required.

7.3. Proposition. Let $M$ be a hyperbolic 3-manifold and let $\epsilon$ be a Margulis number for $M$. Let $(\Sigma, f, S)$ be an $(\epsilon / 3)$-girded surface of total genus $g$ in $M$. Let $\alpha_{1}, \ldots, \alpha_{k}$ be the distinct geodesics carried by $(\Sigma, f, S)$, and let $\mathcal{T}_{i}$ denote the component of $M_{(0, \epsilon]}$ containing $\alpha_{i}$. Then $f(\Sigma) \subset \operatorname{nbhd}_{D}\left(\mathcal{T}_{1} \cup \cdots \cup \mathcal{T}_{k}\right)$, where $D=D(g, \epsilon / 3)$ in the notation of Proposition 6.4.

Proof. In the metric defined by the PH structure of $\Sigma$ induced by $f$, we have by Proposition 6.4 that the diameter of $\Sigma$ modulo $\Sigma_{(0, \epsilon / 3]}$ is less than $D=D(g, \epsilon / 3)$.

Thus $\Sigma=\operatorname{nbhd}_{D}\left(\Sigma_{(0, \epsilon / 3]}\right)$. Since $f$ is distance-decreasing it follows that $f(\Sigma) \subset$ $\operatorname{nbhd}_{D}\left(f\left(\Sigma_{(0, \epsilon / 3]}\right)\right)$. In order to prove the proposition, it therefore suffices to show that $f\left(\Sigma_{(0, \epsilon / 3]}\right) \subset \mathcal{T}_{1} \cup \cdots \cup \mathcal{T}_{k}$.

Let $x$ be any point of $\Sigma_{(0, \epsilon / 3]}$. Then there is a closed curve $\gamma: S^{1} \rightarrow \Sigma$ which passes through $x$, is homotopically non-trivial in $\Sigma$ and has length $<\epsilon / 3$. If $\gamma\left(S^{1}\right)$ intersects $S$ then $x$ is in the $\epsilon / 3$-neighborhood of $S$; since $f$ is distance-decreasing it follows that $f(x) \in \operatorname{nbhd}_{\epsilon / 3}\left(\alpha_{i}\right)$ for some $i$. Since the closed geodesic $\alpha_{i}$ has length $<\epsilon / 3$ it is clear that $\operatorname{nbhd}_{\epsilon / 3}\left(\alpha_{i}\right)$ is contained in $M_{(0, \epsilon]}$ and hence in $\mathcal{T}_{i}$; thus $f(x) \in \mathcal{T}_{i}$ in this case.

Finally, suppose that $\gamma\left(S^{1}\right)$ is disjoint from $S$. Then $\gamma$ is contained in some component $R$ of $\Sigma-S$. Since $\gamma$ is homotopically non-trivial in $\Sigma$, the graph $\mathcal{G}=\gamma\left(S^{1}\right)$ is non-contractible in $R$ (i.e. the inclusion $\mathcal{G} \rightarrow R$ is not homotopic to a constant). Hence $\mathcal{G}$ contains a simple closed curve $C^{\prime}$ which is non-contractible in $R$. By condition (ii) in the definition of an $\epsilon$-girded surface, $R$ is homeomorphic to a sphere with three punctures, and hence $C^{\prime}$ is parallel in $\Sigma$ to a component $C$ of $S$. Since $\gamma$ has length $<\epsilon / 3$, the curve $C^{\prime}$ has length $<\epsilon / 3$, and $x$ lies in the $\epsilon / 3$-neighborhood of $C^{\prime}$. Hence there is a loop $\gamma^{\prime}$ based at $x$ which is freely homotopic to the simple closed curve $C$ and has length $<\epsilon$. Now $f \circ \gamma^{\prime}$ has length $<\epsilon$ and is freely homotopic to $\alpha_{i}$. Hence $f\left(\gamma^{\prime}\left(S^{1}\right)\right) \subset \mathcal{T}_{i}$. In particular $f(x) \in \mathcal{T}_{i}$.

7.4. Girded manifolds. An $\epsilon$-girded surface is said to enclose a set if its underlying hyperbolically simplicial surface is null-homologous and encloses the set. Let $M$ be a complete hyperbolic 3-manifold. We shall say that $M$ is girded if there exist an integer $g$ and a point $P_{0} \in M$ such that for every $\epsilon>0$ there is an $\epsilon$-girded surface of total genus at most $g$ which encloses $P_{0}$. A Kleinian group $\Gamma$ will be termed girded if the hyperbolic 3-manifold $M(\Gamma)$ is girded.

Proposition. If a complete hyperbolic 3-manifold $M$ is girded then every positive superharmonic function on $M$ is constant.

Proof. Let $M$ be a complete hyperbolic 3-manifold and let $\epsilon$ be a Margulis constant for $M$. 
We will show that every compact set in $M$ is enclosed by an $\epsilon / 3$ - girded surface of total genus at most $g$. Any $\epsilon / 3$-girded surface is $\epsilon / 3$-incompressible by Proposition 7.2 and is by definition a Bonahon surface. The proposition then follows immediately from Proposition 6.8 .

Let $K$ be a compact set in $M$. By enlarging $K$ if necessary we may assume that $K$ is connected and that $P_{0} \in K$. Set $D=D(g, \epsilon / 3)$, in the notation of Proposition 6.4. Since $\operatorname{nbhd}_{D}(K)$ is compact there are only finitely many components of $M_{(0, \epsilon]}$ which meet $\operatorname{nbhd}_{D}(K)$. Each of these is a tube around a geodesic of length less than $\epsilon$. Let $\epsilon^{\prime}<\epsilon / 3$ be a positive number which is less than the length of the core geodesic of any of the components of $M_{(0, \epsilon]}$ which meets $\operatorname{nbhd}_{D}(K)$. Thus if $\alpha$ is a geodesic of length less than $\epsilon^{\prime}$ then the component $\mathcal{T}$ of $M_{(0, \epsilon]}$ which contains $\alpha$ is disjoint from $\operatorname{nbhd}_{D}(K)$; equivalently, $\operatorname{nbhd}_{D}(\mathcal{T})$ is disjoint from $K$.

Now it follows from Proposition 7.3 that any $\epsilon^{\prime}$-girded surface is disjoint from $K$. Let $(\Sigma, f, S)$ be an $\epsilon^{\prime}$-girded surface of total genus at most $g$ that encloses the point $P_{0}$. Since the set of points of $M$ which are enclosed by $(\Sigma, f, S)$ is open and closed in $M-f(\Sigma)$ and since $K$ is connected and contains $P_{0}$ it follows that $(\Sigma, f, S)$ encloses $K$. An $\epsilon^{\prime}$-girded surface is also $\epsilon / 3$-girded. Thus we have constructed an $\epsilon / 3$-girded surface of total genus at most $g$ that encloses $K$, as desired.

\section{$\S 8$. GiRded GROUPS ARE DENSE}

8.1. We shall let $F$ denote the abstract free group on two generators $x$ and $y$. We fix an identification of the group of orientation-preserving isometries of $\mathbf{H}^{3}$ with $\mathrm{PSL}_{2}(\mathbf{C})$. The representations of $F$ in $\mathrm{PSL}_{2}(\mathbf{C})$ are in 1-1 correspondence with points of the complex affine variety $V=\mathrm{PSL}_{2}(\mathbf{C}) \times \mathrm{PSL}_{2}(\mathbf{C})$; the representation $\rho=\rho_{\xi, \eta}$ corresponding to a point $(\xi, \eta)$ of $V$ is defined by $\rho(x)=\xi, \rho(y)=\eta$. We shall always understand $V$ to be endowed with the complex topology. We shall denote by $\mathfrak{D}$ the set of all points $(\xi, \eta) \in V$ such that $\rho_{\xi, \eta}$ is a faithful representation with discrete image, i.e. such that the group $\langle\xi, \eta\rangle$ generated by $\xi$ and $\eta$ is a Kleinian group which is free of rank 2. According to [Ch], $\mathfrak{D}$ is a closed subset of $V$. We shall denote by $\mathfrak{G} \mathfrak{F}$ the set of all points $(\xi, \eta) \in \mathfrak{D}$ such that $\langle\xi, \eta\rangle$ is a geometrically finite group (1.7) without parabolic elements. It follows from [Mar, Theorem 8.1] that $\mathfrak{G} \mathfrak{F}$ is an open subset of $V$. We shall denote by $\mathfrak{B}$ the frontier of $\mathfrak{G} \mathfrak{F}$ in $V$; thus $\mathfrak{B}=\overline{\mathfrak{G} \mathfrak{F}}-\mathfrak{G} \mathfrak{F}$.

8.2. The purpose of this section is to prove the following result.

Theorem. There is a dense $G_{\delta}$-set $\mathfrak{C} \subset \mathfrak{B}$ such that for every $(\xi, \eta) \in \mathfrak{C}$, the Kleinian group $\langle\xi, \eta\rangle$ is girded.

8.3. Note that for any $(\xi, \eta) \in \mathfrak{B}$, the hyperbolic 3 -manifold $M(\langle\xi, \eta\rangle)=\mathbf{H}^{3} /\langle\xi, \eta\rangle$ satisfies $H_{2}(M(\langle\xi, \eta\rangle) ; \mathbf{Z} / 2) \cong H_{2}(F ; \mathbf{Z} / 2)=0$ since $F$ is free. Thus every hyperbolically simplicial surface in $M(\langle\xi, \eta\rangle)$ is null-homologous. In order to prove that $\langle\xi, \eta\rangle$ is girded for a given $(\xi, \eta) \in \mathfrak{B}$, it suffices to show that for every $\epsilon>0$, every compact subset of $M(\langle\xi, \eta\rangle)$ is enclosed by some connected $\epsilon$-girded surface of genus 2 .

8.4. Since the details of the of the proof of Theorem 8.2 are rather involved, we begin with a rough sketch of the argument. There are two main issues. First one must produce, for a 
generic point $(\xi, \eta) \in \mathfrak{B}$ and an arbitrary $\epsilon>0$, a connected $\epsilon$-girded surface of genus 2 in the hyperbolic 3-manifold $M=M(\langle\xi, \eta\rangle)$. Second one must choose this girded surface to enclose a prescribed point in $M$.

To deal with the first issue we observe that an $\epsilon$-girded surface of genus 2 determines three closed geodesics of length $<\epsilon$ in $M$; since these geodesics are defined by simple closed curves in the domain surface, their homotopy classes are of a restricted type. Conversely, if one can realize a suitable triple of homotopy classes by a triple of geodesics of length $<\epsilon$, one can construct an $\epsilon$-girded genus 2 surface in $M$. That such a triple of geodesics exists for a generic $(\xi, \eta)$ will be deduced from a recent result due to Curt McMullen, asserting the density of "maximal cusps" in $\mathfrak{B}$ (see Theorem 8.9 below).

To deal with the second issue, we choose our $\epsilon$-girded surfaces to be equivariant with respect to suitable involutions of $M$ and of the domain surface. We then apply Proposition 6.10 to conclude that our girded surfaces enclose appropriately defined "reference points" in $M$. The existence of the appropriate involution of $M$ follows from the results presented in 1.8-1.15. This stage of the argument depends strongly on the hypothesis that $F$ is a 2 -generator group.

8.5. For the rest of the section we fix, arbitrarily, a base point $w_{0} \in \mathbf{H}^{3}$. We shall let $p_{\xi, \eta}$ denote the covering projection from $\mathbf{H}^{3}$ to $M(\langle\xi, \eta\rangle)$.

For any $(\xi, \eta) \in \mathfrak{D}$ the group $\langle\xi, \eta\rangle$ is free of rank 2. In particular $\langle\xi, \eta\rangle$ is not solvable, and hence $\xi$ and $\eta$ have no common fixed point. It follows by 1.8 that each $(\xi, \eta) \in \mathfrak{D}$ determines a line $\ell(\xi, \eta)$ in $\mathbf{H}^{3}$. We shall write $\tau_{\xi, \eta}=\tau_{\ell(\xi, \eta)}$; by 1.8 , the involution $\tau=\tau_{\xi, \eta}$ satisfies $\tau \xi \tau=\xi^{-1}$ and $\tau \eta \tau=\eta^{-1}$. Since $\tau_{\xi, \eta}$ normalizes $\langle\xi, \eta\rangle$, it induces an involution $T_{\xi, \eta}$ of $M(\langle\xi, \eta\rangle)$.

We shall denote by $\widehat{F}$ the group defined by the presentation

$$
\left\langle x, y, t \mid t^{2}=1, t x t=x^{-1}, t y t=y^{-1}\right\rangle .
$$

We may regard $\widehat{F}$ as a semi-direct product of the free group $F=\langle x, y\rangle$ by the cyclic group $\left\langle t \mid t^{2}=1\right\rangle$. Thus $F$ may be identified with an index-2 subgroup of $\widehat{F}$. On the other hand, if we rewrite the presentation for $\widehat{F}$ in terms of the generators $t_{1}=t, t_{2}=t x$ and $t_{3}=t y$, we obtain $\left\langle t_{1}, t_{2}, t_{3} \mid t_{1}^{2}=t_{2}^{2}=t_{3}^{2}=1\right\rangle$, so that $\widehat{F}$ is a free product of three groups of order 2. It follows (for example by the Kurosh subgroup theorem [K, p. 34]) that every element of finite order in $\widehat{F}$ is conjugate to one of the $t_{j}$.

For every point $(\xi, \eta) \in \mathfrak{D}$ the representation $\rho_{\xi, \eta}: F \rightarrow \mathrm{PSL}_{2}(\mathbf{C})$ extends to a representation $\widehat{\rho}=\widehat{\rho}_{\xi, \eta}: F \rightarrow \mathrm{PSL}_{2}(\mathbf{C})$ defined by $\widehat{\rho}(x)=\xi$, $\widehat{\rho}(y)=\eta, \widehat{\rho}(t)=\tau_{\xi, \eta}$. The index of $\rho_{\xi, \eta}(F)=\langle\xi, \eta\rangle$ in $\widehat{F}_{\xi, \eta}=\widehat{\rho}_{\xi, \eta}(\widehat{F})=\left\langle\xi, \eta, \tau_{\xi, \eta}\right\rangle$ is exactly 2 ; indeed, the index is at most 2 since $\widehat{F}$ has index 2 in $F$, and we cannot have $F_{\xi, \eta}=\widehat{F}_{\xi, \eta}$ since $F_{\xi, \eta}$ is free and $\widehat{F}_{\xi, \eta}$ contains an element of order 2. It follows that $\widehat{\rho}$ is a faithful representation with discrete image.

Thus $\widehat{F}_{\xi, \eta}$ is a Kleinian group having exactly three conjugacy classes of elements of finite order. Hence the set $\mathcal{L}(\xi, \eta)$ of fixed points of elements of $\widehat{F}_{\xi, \eta}$ is the union of a locally finite disjoint family of lines in $\mathbf{H}^{3}$, each of which is the fixed point set of a unique element of $\widehat{F}_{\xi, \eta}$; and this family consists of exactly three $\widehat{F}_{\xi, \eta}$-orbits of lines. Hence Fix $T_{\xi, \eta}=p_{\xi, \eta}(\mathcal{L}(\xi, \eta))$ is a disjoint union of three geodesics in $M(\langle\xi, \eta\rangle)$. 
Explicitly, the three conjugacy classes of elements of finite order in $\widehat{F}_{\xi, \eta}$ are represented by the elements $\tau_{\xi, \eta, j}=\widehat{\rho}_{\xi, \eta}\left(t_{j}\right)$ for $j=1,2,3$. We have $\tau_{\xi, \eta, 1}=\tau_{\xi, \eta}, \tau_{\xi, \eta, 2}=\tau_{\xi, \eta} \xi$ and $\tau_{\xi, \eta, 3}=\tau_{\xi, \eta} \eta$. We set $\ell_{j}(\xi, \eta)=\operatorname{Fix} \tau_{\xi, \eta, j}$. We have $\ell_{1}(\xi, \eta)=\ell(\xi, \eta)$. The components of Fix $T_{\xi, \eta}$ are $p_{\xi, \eta}\left(\ell_{j}(\xi, \eta)\right)$ for $j=1,2,3$. We shall write $L_{j}(\xi, \eta)=p_{\xi, \eta}\left(\ell_{j}(\xi, \eta)\right)$.

8.6. Proposition. For any $(\xi, \eta) \in \mathfrak{D}$ and for $j=1,2,3$, the line $\ell_{j}(\xi, \eta)$ is mapped homeomorphically onto $L_{j}(\xi, \eta)$ by $p_{\xi, \eta}$.

Proof. Since $L_{j}(\xi, \eta)$ is a component of Fix $T_{\xi, \eta}$, it is a closed subset of $M(\langle\xi, \eta\rangle)$. Hence if the lemma were false, $L_{j}(\xi, \eta)$ would be a closed geodesic, and $\ell_{j}(\xi, \eta)$ would be the axis of a loxodromic element $\gamma$ of $F$. Let us set $\ell=\ell_{j}(\xi, \eta)$ and $\tau=\tau_{\xi, \eta, j}$. Then $\tau$ fixes $\ell$ (pointwise) and hence so does the commutator $[\tau, \gamma] \in \widehat{F}$. Since $\widehat{F}$ is discrete it follows that $[\tau, \gamma]$ has finite order. But the commutator subgroup of $\widehat{F} \cong \mathbf{Z}_{2} * \mathbf{Z}_{2} * \mathbf{Z}_{2}$ is torsion-free, and hence $\gamma$ must commute with $\tau$. But the centralizer of $\tau$ in $\widehat{F}$ has order 2 (for example by the Kurosh subgroup theorem) and $\gamma$ has infinite order; this is a contradiction.

8.7. We let $\widetilde{P}_{j}(\xi, \eta)$ denote the point of $\ell_{j}(\xi, \eta)$ which is closest to the base point $w_{0}$, and we set $P_{j}(\xi, \eta)=p_{\xi, \eta}(\widetilde{P}(\xi, \eta)) \in L_{j}(\xi, \eta)$. The points $P_{j}(\xi, \eta), j=1,2,3$, will be called the reference points of $M(\langle\xi, \eta\rangle)$. Thus $M(\langle\xi, \eta\rangle)$ contains exactly three reference points, and there is one reference point in each component of $\mathrm{Fix} T_{\xi, \eta}$.

8.8. We shall let $\mathfrak{A}$ denote the set of all points $(\xi, \eta) \in \mathfrak{D}$ such that the group $\langle\xi, \eta\rangle$ contains no parabolics. The following result, which will be needed for the proof of Theorem 8.2, was announced in [Ber1]. A version of Bers's $\lambda$-lemma can be used to complete the proof: see [McM, Corollary 1.5].

Proposition. The set $\mathfrak{A} \cap \mathfrak{B}$ is a dense $G_{\delta}$ in $\mathfrak{B}$.

8.9. The following result, due to Curt McMullen, is the deepest ingredient in the proof of Theorem 8.2. McMullen has informed us that the theorem below is proved by a generalization of the techniques of $[\mathrm{McM}]$.

Theorem. There is a dense subset $\mathfrak{E}$ of $\mathfrak{B}$ such that for every $(\xi, \eta) \in \mathfrak{E}$, the free Kleinian group $\langle\xi, \eta\rangle$ is geometrically finite and has three distinct conjugacy classes of cuspidal subgroups (1.5).

8.10. The proof of Theorem 8.2 will be proved by combining the results stated above with the following result.

Proposition. Let $(\alpha, \beta)$ be a point of $\mathfrak{D}$ such that $\langle\alpha, \beta\rangle$ is geometrically finite and contains three distinct conjugacy classes of cuspidal subgroups. Let $\epsilon$ be any positive number. Then $(\alpha, \beta)$ has a neighborhood $U=U_{\epsilon}(\alpha, \beta)$ in $\mathfrak{B}$ such that for every $(\xi, \eta) \in$ $\mathfrak{A} \cap U$, there is a connected $\epsilon$-girded surface of genus 2 in $M(\langle\xi, \eta\rangle)$ which encloses at least one of the reference points of $M(\langle\xi, \eta\rangle)$.

8.11. Before proving Proposition 8.10, let us give the

Proof that Proposition 8.10 implies Theorem 8.2. According to Theorem 8.9 there is a dense set $\mathfrak{E} \subset \mathfrak{B}$ such that for every $(\alpha, \beta) \in \mathfrak{E}$, the group $\langle\alpha, \beta\rangle$ is geometrically finite and has three non-conjugate maximal cuspidal subgroups. For each $\epsilon>0$ and each $(\alpha, \beta) \in \mathfrak{E}$, 
Proposition 8.10 gives a neighborhood $U=U_{\epsilon}(\alpha, \beta)$ of $(\alpha, \beta)$ in $\mathfrak{B}$. For every $\epsilon>0$ the set

$$
W_{\epsilon}=\bigcup_{(\alpha, \beta) \in \mathfrak{E}} U_{\epsilon}(\alpha, \beta)
$$

is open and dense in $\mathfrak{B}$. On the other hand, by Proposition $8.8, \mathfrak{A} \cap \mathfrak{B}$ is a dense $G_{\delta}$ in $\mathfrak{B}$. Hence the set

$$
\mathfrak{C}=\mathfrak{A} \cap \bigcap_{0<n \in \mathbf{Z}} W_{1 / n}
$$

is a dense $G_{\delta}$ in $\mathfrak{B}$.

To complete the proof it suffices to show that for every $(\xi, \eta) \in \mathfrak{C}$ the group $\langle\xi, \eta\rangle$ is girded. If $(\xi, \eta) \in \mathfrak{C}$ then for every positive integer $n$ there is a point $\left(\alpha_{n}, \beta_{n}\right) \in \mathfrak{E}$ such that $(\xi, \eta) \in \mathfrak{A} \cap U_{1 / n}\left(\alpha_{n}, \beta_{n}\right)$. According to the defining property of $U_{\epsilon}\left(\alpha_{n}, \beta_{n}\right)$ (see Proposition 8.10) this means that for every $n>0$ there is a reference point $z_{j_{n}}$ of $M(\langle\xi, \eta\rangle)$ which is enclosed by a connected $(1 / n)$-girded surface of genus 2 in $M(\langle\xi, \eta\rangle)$.

Since $M(\langle\xi, \eta\rangle)$ has only three reference points, it follows that there is a single reference point $z$ of $M$ which is enclosed by a connected $(1 / n)$-girded surface of genus 2 for arbitrarily large values of $n$; it follows at once that $M(\langle\xi, \eta\rangle)$ is girded.

8.12. The rest of this section is devoted to the proof of Proposition 8.10. We suppose that we are given a point $(\alpha, \beta)$ of $\mathfrak{D}$ such that the free Kleinian group $\langle\alpha, \beta\rangle$ is geometrically finite and contains three distinct conjugacy classes of cuspidal subgroups. We set $\widehat{\rho}_{0}=\widehat{\rho}_{\alpha, \beta}$, $p_{0}=p_{\alpha, \beta}: \mathbf{H}^{3} \rightarrow M(\langle\alpha, \beta\rangle)$, and $T_{0}=T_{\alpha, \beta}$.

8.13. Lemma. For each component $L$ of Fix $T_{0}$ we have $N(\langle\alpha, \beta\rangle) \cap L \neq \emptyset$. Furthermore, there is a constant $\eta>0$ such that for every $\epsilon<\eta$ and every component $L$ of Fix $T_{0}$ we have $N_{[\epsilon, \infty)}(\langle\alpha, \beta\rangle) \cap L \neq \emptyset$.

Proof. To prove the first assertion we choose a component $\ell$ of $p_{0}^{-1}(L)$. Then $\ell$ is a component of $\mathcal{L}(\alpha, \beta)$, and hence by 8.5 we have $\ell=$ Fix $\tau$ for some order-2 element $\tau$ of $\widehat{\rho}_{0}(\widehat{F})$. Recall from 1.4 that the set $\widetilde{N}(\langle\alpha, \beta\rangle)=p_{0}^{-1}(N(\langle\alpha, \beta\rangle))=\operatorname{nbhd}_{1}\left(\operatorname{hull}\left(\Lambda_{\langle\alpha, \beta\rangle}\right)\right)$ is $\langle\alpha, \beta\rangle$-invariant and convex. Since $\tau$ normalizes $\langle\alpha, \beta\rangle$ (see 8.5), $\widetilde{N}(\langle\alpha, \beta\rangle)$ is $\tau$-invariant.

It follows that for any point $z \in \widetilde{N}(\langle\alpha, \beta\rangle)$, the midpoint of the line segment joining $z$ to $\tau z$ lies in $N(\langle\alpha, \beta\rangle) \cap L$, and the first assertion is proved.

It follows from the first assertion that for any component $L$ of Fix $T_{0}$ there is a number $\eta_{L}>0$ such that $N_{\left[\eta_{L}, \infty\right)}(\langle\alpha, \beta\rangle) \cap L \neq \emptyset$. The second assertion now follows if we set $\eta=\min _{L} \eta_{L}$, where $L$ ranges over the three components of Fix $T_{0}$.

8.14. Let $\epsilon_{0}$ be a 3-dimensional Margulis constant such that conclusions (i) and (ii) of Proposition 1.7 hold with $\Gamma=\langle\alpha, \beta\rangle$. According to 1.7 we may take $\epsilon_{0}$ to be arbitrarily small. In particular we may suppose $\epsilon_{0}$ to be chosen so that $N_{\left[\epsilon_{0}, \infty\right)}(\langle\alpha, \beta\rangle) \cap L \neq \emptyset$ for every component $L$ of Fix $T_{0}$.

By conclusion (i) of 1.7, $J=N_{\left[\epsilon_{0}, \infty\right)}(\langle\alpha, \beta\rangle)$ is a compact, orientable, irreducible 3manifold-with-boundary. Furthermore, $J$ is a deformation retract of $M(\langle\alpha, \beta\rangle)$; in particular $J$ is connected and $\pi_{1}(J)$ is a free group of rank 2. According to [He, Theorem 5.2], any compact, connected, orientable, irreducible 3-manifold-with-boundary whose fundamental group is free of rank 2 is a genus- 2 handlebody. 
We set $\Sigma=\partial J$. Then $\Sigma$ is a closed orientable surface of genus 2 . Since the fundamental group of a handlebody is carried by its boundary, and since $J$ is a deformation retract of $M(\langle\alpha, \beta\rangle)$, the inclusion homomorphism $\pi_{1}(\Sigma) \rightarrow \pi_{1}(M(\langle\alpha, \beta\rangle))$ is surjective.

Hence $\widetilde{\Sigma}=p_{0}^{-1}(\Sigma)$ is a connected covering surface of $\Sigma$. Clearly $\widetilde{\Sigma}$ is invariant under the action of $\widehat{F}$ on $\mathbf{H}^{3}$ defined by the representation $\rho_{0}$. Restricting this action, we obtain a natural action of $\widehat{F}$ on $\widetilde{\Sigma}$.

Since the group $\langle\alpha, \beta\rangle$ contains three conjugacy classes of cuspidal subgroups, the set $M_{\left(0, \epsilon_{0}\right]}^{c}(\langle\alpha, \beta\rangle)$ has three components. Each component of $M_{\left(0, \epsilon_{0}\right]}^{c}(\langle\alpha, \beta\rangle)$ has the form $C / \Pi$, where $\Pi$ is a cuspidal subgroup of $\Gamma$ and $C$ is a horoball stabilized by $\Pi$. Since $\langle\alpha, \beta\rangle$ is free, each of these groups $\Pi$ is cyclic. Thus $M_{\left(0, \epsilon_{0}\right]}(\langle\alpha, \beta\rangle)$ has the homotopy type of a disjoint union of three circles. Hence if we set $\mathcal{A}=N_{\left\{\epsilon_{0}\right\}}(\langle\alpha, \beta\rangle)$, it follows from conclusion (ii) of 1.7 that $\mathcal{A}$ is a disjoint union of three annuli in $\Sigma$, all homotopically non-trivial in $M(\langle\alpha, \beta\rangle)$.

We set $\widetilde{\mathcal{A}}=p_{0}^{-1}(\mathcal{A}) \subset \widetilde{\Sigma} \subset \mathbf{H}^{3}$. Each component $\widetilde{A}$ of $\widetilde{\mathcal{A}}$ is contained in a unique horoball in $\mathbf{H}^{3}$, which we denote by $C(\widetilde{A})$. We denote the base point of $C(\widetilde{A})$ in $S_{\infty}$ by $\zeta(\widetilde{A})$. The stabilizer of $\widetilde{A}$ in $\langle\xi, \eta\rangle$ is an infinite cyclic group consisting of parabolic elements, and this group leaves $C(\widetilde{A})$ invariant and fixes $\zeta(\widetilde{A})$.

Since the three annuli comprising $\mathcal{A}$ correspond to different conjugacy classes of (cuspidal) maximal infinite cyclic subgroups of $\Gamma$, their cores are non-contractible and (freely) non-homotopic simple closed curves in $M(\langle\alpha, \beta\rangle)$. In particular they are non-contractible and non-homotopic in $\Sigma$. Hence each component of $\overline{\Sigma-\mathcal{A}}$ is a planar surface with three boundary curves, and at least two of the components of $\mathcal{A}$ are non-separating annuli in $\Sigma$.

It is apparent from the definitions of $J$ and $\mathcal{A}$ that they are invariant under any selfisometry of $M(\langle\alpha, \beta\rangle)$. Thus in particular $J$ is invariant under $T_{0}$, so that $T_{0}$ induces an involution $T_{\Sigma}$ of $\Sigma$; and $\mathcal{A}$ is invariant under $T_{\Sigma}$. (We shall sharpen this assertion in Lemma 8.16 below.)

Observe also that since $T_{0}$ is induced by an orientation-preserving isometry of $\mathbf{H}^{3}$, it is itself orientation-preserving. It follows that $T_{0} \mid J$ is orientation-preserving, and therefore so is $T_{\Sigma}$.

8.15. Lemma. For every component $L$ of $\operatorname{Fix} T_{0}$ we have $\operatorname{card}(L \cap \Sigma)=2$.

Proof. Recall from 8.14 that we chose $\epsilon_{0}$ in such a way that $J \cap L=N_{\left[\epsilon_{0}, \infty\right)}(\langle\alpha, \beta\rangle) \cap L \neq \emptyset$ for every component $L$ of Fix $T_{0}$. Since $J \cap L$ is a union of components of the fixed set of the involution $T_{0} \mid J$ of the compact 3-manifold-with-boundary $J$, it is a properly embedded 1-manifold-with-boundary in $J$; it has no closed components since $L$ is homeomorphic to $\mathbf{R}$ by 8.6. Thus for each component $L$ of Fix $T_{0}$, the set $\Sigma \cap L=\partial(J \cap L)$ has non-zero, finite, even cardinality. On the other hand, the sum of the cardinalities of $\Sigma \cap L$ as $L$ ranges over the three components of $\operatorname{Fix} T_{0}$ is equal to card Fix $T_{\Sigma}$, the number of fixed points of an orientation-preserving involution of a closed connected surface, which by the Hurwitz branching formula is at most 6 . Hence we must have card $(\Sigma \cap L)=2$ for each component $L$ of Fix $T_{0}$.

8.16. Lemma. Each component of $\mathcal{A}$ is invariant under $T_{\Sigma}$.

Proof. If the assertion is false then two components $A_{1}$ and $A_{2}$ are interchanged by $T_{\Sigma}$. 
Since $\mathcal{A}$ has at least two non-separating components, $A_{1}$ and $A_{2}$ cannot both separate $\Sigma$; since they are interchanged by the homeomorphism $T_{\Sigma}$, they are both non-separating. Hence $\Sigma^{\prime}=\overline{\Sigma-\left(A_{1} \cup A_{2}\right)}$ is connected. Since neither $A_{1}$ nor $A_{2}$ can contain a fixed point of $T_{\Sigma}$, and since $T_{\Sigma}$ has six fixed points by Lemma 8.15, $T_{\Sigma^{\prime}}=T_{\Sigma} \mid \Sigma^{\prime}$ is an orientation-preserving involution, with six fixed points, of a connected surface having nonempty boundary and Euler characteristic -2. It then follows from the Hurwitz branching formula that $\Sigma^{\prime} / T_{\Sigma^{\prime}}$ is a connected surface having non-empty boundary and Euler characteristic 2 . But no such surface exists.

8.17. It follows from Lemma 8.16 that for each component $A$ of $\mathcal{A}$ we may choose a $T_{\Sigma^{-}}$ invariant core curve $S_{A}$. Having made such a choice, we set $S=\bigcup_{A} S_{A}$, where $A$ ranges over the components of $\mathcal{A}$. It follows from 8.14 that the $S_{A}$ are homotopically non-trivial curves in $M(\langle\alpha, \beta\rangle)$, that at least two of the $S_{A}$ are non-separating curves in $\Sigma$, and that each component of $\Sigma-S$ is a three-punctured sphere. We set $S=p_{0}^{-1}(S) \subset \widetilde{\Sigma}$.

Lemma. For each component $R$ of $\Sigma-S$, the inclusion homomorphism from $\pi_{1}(R)$ to $\pi_{1}(M(\langle\alpha, \beta\rangle))$ is injective.

Proof. Since $J$ is a deformation retract of $M(\langle\alpha, \beta\rangle)$ by 8.14 , it suffices to show that the inclusion homomorphism $\pi_{1}(R) \rightarrow \pi_{1}(J)$ is injective. If it is not, it follows from the loop theorem [He, Theorem 4.2] that some non-trivial element of $\operatorname{ker}\left(\pi_{1}(R) \rightarrow \pi_{1}(J)\right)$ is represented by a simple closed curve in $R$. Since $R$ is a three-punctured sphere, every simple closed curve in $R$ is parallel to a component of $\partial R \subset S$. But every component of $S$ is homotopically non-trivial in $M(\langle\alpha, \beta\rangle)$ and hence in $J$.

8.18. Lemma. Every non-separating component of $S$ meets $\operatorname{Fix} T_{\Sigma}$ in two points. If $C$ is a separating component of $S$ then $C$ is disjoint from Fix $T_{\Sigma}$, and each component of $\Sigma-C$ is $T_{\Sigma}$-invariant.

Proof. It follows from Lemmas 8.15 and 8.16 that $T_{\Sigma}$ is an orientation-preserving involution of $\Sigma$ with six fixed points and that each component of $S$ is invariant under $T_{\Sigma}$. The Hurwitz branching formula implies that the orbit surface $\Psi=\Sigma / T_{\Sigma}$ is a sphere. Let $q: \Sigma \rightarrow \Psi$ denote the quotient map.

If $C$ is any component of $S$, either $C$ contains two fixed points of $T_{\Sigma}$ and $T_{\Sigma}$ reverses the orientation of $C$, or $C$ contains no fixed point of $T_{\Sigma}$ and $T_{\Sigma}$ preserves the orientation of $C$. If $C$ separates $\Sigma$ then since the other components of $S$ are $T_{\Sigma}$-invariant, each component of $\Sigma-C$ must be $T_{\Sigma}$-invariant; hence $T_{\Sigma}$ preserves the orientation of $C$. Conversely, if $T_{\Sigma}$ preserves the orientation of $C$ then $q(C)$ is a simple closed curve and therefore separates $\Psi$. Hence $C=q^{-1}(q(C))$ separates $\Sigma$.

8.19. Lemma. There is a $T_{\Sigma}$-invariant virtual triangulation of $\Sigma$ such that

(i) $\operatorname{skel}_{0}(\Sigma) \subset S$,

(ii) $S \cup \operatorname{Fix} T_{\Sigma} \subset \operatorname{skel}_{1}(\Sigma)$, and

(iii) no virtual 2-simplex has more than one edge supported in $S$.

Proof. Let us label the components of $S$ as $C_{1}, C_{2}$ and $C_{3}$, where $C_{1}$ and $C_{2}$ are nonseparating curves in $\Sigma$, and $C_{3}$ may or may not separate $\Sigma$. 
Consider the case in which $C_{3}$ is non-separating. Then $\Sigma-S$ has two components $Y_{1}$ and $Y_{2}$, each of which is the interior of a compact planar sub-surface of $\Sigma$ with three boundary curves. Since the $C_{i}$ are non-separating, $T_{\Sigma}$ reverses the orientation of $C_{i}$; hence $T_{\Sigma}$ interchanges $Y_{1}$ and $Y_{2}$. By Lemma 8.18, each $C_{i}$ contains two fixed points of $T_{\Sigma}$. Thus Fix $T_{\Sigma}$ is a subset of $\partial \overline{Y_{1}}$ containing exactly two points of each component of $\partial \overline{Y_{1}}$. It follows (see the figure) that $\overline{Y_{1}}$ has a virtual triangulation with Fix $T_{\Sigma}$ as its 0 -skeleton, and in which no virtual 2-simplex has more than one edge supported in $\partial \overline{Y_{1}}$.

The induced triangulation of $\partial \overline{Y_{1}}$ is automatically $T_{\Sigma}$-invariant, and hence the virtual triangulation of $\overline{Y_{1}}$ extends to a unique $T_{\Sigma}$-invariant virtual triangulation of $\Sigma$; this extended triangulation clearly has the required properties.

Now consider the case in which $C_{3}$ separates $\Sigma$. For $i=1,2$, let $J_{i}$ denote the component of $\Sigma-C_{3}$ containing $C_{i}$. As in the proof of Lemma 8.18 we observe that $\Psi=\Sigma / T_{\Sigma}$ is a sphere and we let $q: \Sigma \rightarrow \Psi$ denote the quotient map. Since $J_{i}$ is $T_{\Sigma}$-invariant by Lemma $8.16, J_{i}$ is a 2 -fold branched cover of the disk $q\left(J_{i}\right) \subset \Psi$. But $J_{i}$ is a genus- 1 surface with connected boundary, and it therefore follows from the Hurwitz branching formula that $J_{i}$ contains exactly three fixed points of $T_{\Sigma}$. Hence there is a unique fixed point $u_{i}$ of $T_{\Sigma}$ lying in $J_{i}-C_{i}$. Now let $v$ be any point of $C_{3}$. For $i=1,2$, there is a $T_{\Sigma}$-invariant, properly embedded arc $\alpha_{i}$ in $J_{i}$ which has $v$ and $T_{\Sigma}(v)$ as endpoints and $u_{i}$ as an interior point: we may in fact define $\alpha_{i}=q^{-1}\left(a_{i}\right)$, where $a_{i}$ is an arc in $q\left(J_{i}\right)$ joining $q(v)$ to $q\left(u_{i}\right)$. If $\alpha_{i}$ separates $J_{i}$, then since $T_{\Sigma}$ clearly reverses the orientation of $\alpha_{i}$, it must interchange the two components of $J_{i}-\alpha_{i}$; this implies that $J_{i}$ has even genus, a contradiction. Hence $\alpha_{i}$ is a non-separating arc in $J_{i}$.

It follows that $C_{3}^{\prime}=\alpha_{1} \cup \alpha_{2}$ is a non-separating simple closed curve in $\Sigma$. Furthermore, since $C_{3}^{\prime}$ intersects each $J_{i}$ in an arc, it cannot be parallel to $C_{1}$ or $C_{2}$. Hence if we set 
$S^{\prime}=C_{1} \cup C_{2} \cup C_{3}^{\prime}$, then $\Sigma-S^{\prime}$ has two components $Y_{1}$ and $Y_{2}$, each of which is the interior of a compact planar sub-surface of $\Sigma$ with three boundary curves. Since the components of $S^{\prime}$ are non-separating, $T_{\Sigma}$ reverses the orientation of each component of $S^{\prime}$; hence $T_{\Sigma}$ interchanges $Y_{1}$ and $Y_{2}$. The set

$$
K=\left(\left(\operatorname{Fix} T_{\Sigma}\right) \cap\left(C_{1} \cup C_{2}\right)\right) \cup\left\{v, T_{\Sigma}(v)\right\} \subset \partial \overline{Y_{1}}
$$

contains exactly two points of each component of $\partial \overline{Y_{1}}$. It follows that $\overline{Y_{1}}$ has a virtual triangulation with $K$ as its 0-skeleton, and in which no virtual 2-simplex has more than one edge supported in $\partial \overline{Y_{1}}$. Note also that the arc $a=C_{3} \cap \overline{Y_{1}}$ is non-boundary parallel in $\overline{Y_{1}}$; for otherwise $a$ would be parallel to one of the $\alpha_{i}$, and this $\alpha_{i}$ would separate $J_{i}, a$ contradiction. It follows that we may choose the virtual triangulation of $\overline{Y_{1}}$ in such a way that $a$ is contained in the 1-skeleton. As in the previous case, the induced triangulation of $\partial \overline{Y_{1}}$ is $T_{\Sigma}$-invariant so that the virtual triangulation of $\overline{Y_{1}}$ extends to a unique $T_{\Sigma}$-invariant virtual triangulation of $\Sigma$; and again the extended triangulation clearly has the required properties.

8.20. For the rest of this section we fix a virtual triangulation of $\Sigma$ having the properties stated in Lemma 8.19. Since the virtual triangulation of $\Sigma$ is $T_{\Sigma}$-invariant, it induces an $\widehat{F}$-invariant virtual triangulation of $\widetilde{\Sigma}$. We shall always understand $\widetilde{\Sigma}$ to be equipped with this virtual triangulation.

Let $(\xi, \eta)$ be any point in $\mathfrak{D}$, and let $\widetilde{f}$ be a map of $\widetilde{\Sigma}$ into $\mathbf{H}^{3}$. We shall say that $\widetilde{f}$ is $(\xi, \eta)$-good if (i) $\widetilde{f}$ is hyperbolically simplicial, (ii) $\widetilde{f} \mid \widetilde{S}$ is geodesic and (iii) $\widetilde{f}$ is $\widehat{F}$ equivariant with respect to the standard action of $\widehat{F}$ on $\widetilde{\Sigma}$ and the action on $\mathbf{H}^{3}$ associated to $(\xi, \eta)$. Any $(\xi, \eta)$-good map $\widetilde{f}: \widetilde{\Sigma} \rightarrow \mathbf{H}^{3}$ induces a hyperbolically simplicial map $f: \Sigma \rightarrow M(\langle\xi, \eta\rangle)$, and $f \mid S$ is geodesic. The $\widehat{F}$-equivariance of $\widetilde{f}$ implies that $f$ satisfies $T_{\xi, \eta} \circ f=f \circ T_{\Sigma}$. If $\widetilde{f}$ is non-degenerate (as a hyperbolically simplicial map) then so is $f$.

8.21. Lemma. For every $(\xi, \eta) \in \mathfrak{A}$ there exists a $(\xi, \eta)$-good map $\widetilde{f}=\widetilde{f}_{\xi, \eta}: \widetilde{\Sigma} \rightarrow \mathbf{H}^{3}$.

Proof. It follows from Lemma 8.19 that $\operatorname{skel}_{0}(\widetilde{\Sigma}) \subset \widetilde{S} \subset \operatorname{skel}_{1}(\widetilde{\Sigma})$. The first step in the construction of $\widetilde{f}$ is to construct a geodesic, $\widehat{F}$-equivariant map $\widetilde{f}_{S}: \widetilde{S} \rightarrow \mathbf{H}^{3}$.

Let us denote the components of $S$ by $C_{1}, C_{2}$ and $C_{3}$. For $i=1,2,3$, we choose a component $\widetilde{S}_{i}$ of $p_{0}^{-1}\left(C_{i}\right)$. We let $G_{i}$ and $\widehat{G}_{i}$ denote the stabilizers of $\widetilde{S}_{i}$ in $F$ and $\widehat{F}$ respectively. Then $G_{i}$ is infinite cyclic, and it has index 2 in $\widehat{G}_{i}$ since $C_{i}$ is $T_{\Sigma}$-invariant. Hence $\widehat{G}_{i}$ is either infinite cyclic or infinite dihedral. If $\gamma_{i}$ denotes a generator of $G_{i}$ then $\gamma_{i}$ has infinite order in $F$, and $\gamma_{i}$ is not parabolic since $(\xi, \eta) \in \mathfrak{A}$. Hence $\gamma_{i}$ has a unique axis $Z_{i} \subset \mathbf{H}^{3}$. Since $\widehat{G}_{i}$ normalizes $G_{i}$, the line $Z_{i}$ is $\widehat{G}_{i}$-invariant.

Up to equivariant homeomorphism, each of the groups $\mathbf{Z}$ and $D_{\infty}$ has a unique properly discontinuous action on $\mathbf{R}$. Hence there is a $\widehat{G}_{i}$-equivariant homeomorphism $\widetilde{f}_{i}: \widetilde{S}_{i} \rightarrow Z_{i}$. Using the maps $\widetilde{f}_{i}$ we define $\widetilde{f}_{S}$ as follows. Given any point $u \in \widetilde{S}$ there exist an index $i \in\{1,2,3\}$ and an element $\gamma$ of $\widehat{F}$ such that $\gamma \cdot u \in \widetilde{S}_{i}$; the index $i$ is uniquely determined by $u$, but the element $\gamma$ is not. We wish to define $\widetilde{f}_{S}(u)=\gamma^{-1} \cdot \widetilde{f}_{i}(\gamma \cdot u)$. If $\gamma_{1}$ is another element of $\widehat{F}$ such that $\gamma_{1} \cdot u \in \widetilde{S}_{i}$, then $\delta=\gamma_{1} \gamma^{-1}$ belongs to $\widehat{G}_{i}$, and by the $\widehat{G}_{i}$-equivariance of $\widetilde{f}_{i}$ we have

$$
\gamma_{1}^{-1} \cdot \widetilde{f}_{i}\left(\gamma_{1} \cdot u\right)=\gamma^{-1} \delta^{-1} \cdot \widetilde{f}_{i}(\delta \gamma \cdot u)=\gamma^{-1} \cdot \widetilde{f}_{i}(\gamma \cdot u)
$$


Thus $f_{S}$ is well-defined. It follows readily from the definition that it is $\widehat{F}$-equivariant. The next step is to extend $\widetilde{f}_{\mathcal{S}}$ to an $\widehat{F}$-equivariant map of $\operatorname{skel}_{1}(\widetilde{\Sigma})$ into $\mathbf{H}^{3}$. Let $\varphi: \Delta \rightarrow \widetilde{\Sigma}$ be a virtual 1-simplex in the virtual triangulation of $\widetilde{\Sigma}$ which is not carried by $\widetilde{S}$. Let $f_{\varphi}: \Delta \rightarrow \mathbf{H}^{3}$ denote the map which agrees with $\widetilde{f}_{\mathcal{S}} \circ(\varphi \mid \partial \Delta)$ on the boundary of $\Delta$, and is geodesic in the sense that if we identify $\Delta$ with an interval in $\mathbf{R}$ by any affine homeomorphism then $f_{\varphi}$ becomes a geodesic path (parametrized proportionally to arc length). (In particular, if $\widetilde{f}_{S} \circ(\varphi \mid \partial \Delta)$ maps the two boundary points of $\Delta$ to the same point, then $f_{\varphi}$ is constant.) There is a unique map $\widetilde{f}^{(1)}: \operatorname{skel}_{1}(\widetilde{\Sigma}) \rightarrow \mathbf{H}^{3}$ such that $\widetilde{f}^{(1)} \mid \widetilde{S}=\widetilde{f}_{S}$ and such that for every virtual 1-simplex $\varphi$ in the virtual triangulation of $\widetilde{\Sigma}$ which is not carried by $\widetilde{S}$ we have $\widetilde{f}^{(1)} \circ \varphi=f_{\varphi}$. Since $\widetilde{f}_{S}$ is $\widehat{F}$-equivariant, so is $\widetilde{f}^{(1)}$.

To extend $\widetilde{f}^{(1)}$ to $\widehat{\Sigma}$ we may use the following construction. Let $\varphi: \Delta \rightarrow \widetilde{\Sigma}$ be any 2 -simplex. Let $m$ denote the baycenter of $\Delta$. Let $\delta$ denote the convex hull of $\widetilde{f}^{(1)}\left(\varphi\left(\Delta^{(0)}\right)\right.$, where $\Delta^{(0)}$ denotes the set of vertices of $\Delta$. Then $\delta$ is either a hyperbolic triangle, a hyperbolic line segment or a point. If $\delta$ is a hyperbolic triangle we let $\mu$ denote its centroid $[F$, p. 125]. If $\delta$ is a line segment we define $\mu$ to be its midpoint, and if $\delta$ consists of a single point we define $\mu$ to be this point. Now we define a map $f_{\varphi}: \Delta \rightarrow \delta$ by stipulating that $f_{\varphi} \mid \partial \Delta=f^{(1)} \circ(\varphi \mid \partial \Delta)$, that $f(m)=\mu$, and that for each affine segment $\sigma \subset \Delta$ joining $m$ to a point in $\partial \Delta$, the map $f_{\varphi} \mid \sigma$ be geodesic (in the above sense). Now there is a unique map $\widetilde{f}: \widetilde{\Sigma} \rightarrow \mathbf{H}^{3}$ such that $\widetilde{f} \mid \operatorname{skel}_{1}(\Sigma)=\widetilde{f}^{(1)}$ and such that for every virtual 2-simplex $\varphi$ in the virtual triangulation of $\widetilde{\Sigma}$ we have $\widetilde{f}^{(1)} \circ \varphi=f_{\varphi}$. Since $\widetilde{f}^{(1)}$ is $\widehat{F}$-equivariant, so is $\widetilde{f}$. It is clear from the construction that $\widetilde{f}$ is hyperbolically simplicial and that $\widetilde{f} \mid \widetilde{S}$ is geodesic.

8.22 Lemma. Let $\left(\xi^{i}, \eta^{i}\right)_{i \geq 1}$ be a sequence of points in $\mathfrak{A}$ which converges in $\mathfrak{D}$ to $(\alpha, \beta)$. For each $i \geq 1$ let $\widetilde{f}^{i}: \widetilde{\Sigma} \rightarrow \mathbf{H}^{3}$ be a $\left(\xi^{i}, \eta^{i}\right)$-good map. Let $\widetilde{C}$ be any component of $\widetilde{S}$ and let $\widetilde{A}$ denote the unique component of $\widetilde{\mathcal{A}}$ containing $\widetilde{C}$. Then for any point $z \in \widetilde{S}$ we have $\lim _{i \rightarrow \infty} \widetilde{f}^{i}(z)=\zeta(\widetilde{A})$ in $\overline{\mathbf{H}^{3}}$. Furthermore, if $z$ and $z^{\prime}$ are distinct points of $\widetilde{C}$ and $U$ is any neighborhood of $\zeta(\widetilde{A})$ in $\overline{\mathbf{H}}$, then we have $\widetilde{f}^{i}(z) \neq \widetilde{f}^{i}\left(z^{\prime}\right)$ for every $i$, and for sufficiently large $i$ the line joining $\widetilde{f}^{i}(z)$ to $\widetilde{f}^{i}\left(z^{\prime}\right)$ is contained in $U$.

(The definition of $\zeta(\widetilde{A})$ was given in 8.14.)

Proof. Let $F_{\widetilde{C}}$ denote the stabilizer of $\widetilde{C}$ under the action of $F$ on $\mathbf{H}^{3}$ defined by $\rho_{0}$. By 8.14 and 8.17, $F_{\widetilde{C}}$ is cyclic and is generated by a parabolic element $h$, and $\rho_{0}(h)$ fixes $\zeta=\zeta(\widetilde{A})$. For each $i \geq 1$, since $\widetilde{f}^{i}$ is a $(\xi, \eta)$-good map, $\widetilde{f}^{i}(\widetilde{C})$ is a geodesic which is invariant under $\rho_{\xi^{i}, \eta^{i}}(h)$. Thus $\widetilde{f}^{i}(\widetilde{C})$ is the axis of the isometry $\rho_{\xi} i_{\eta} i(h)$ (which is loxodromic since $\left.\left(\xi^{i}, \eta^{i}\right) \in \mathfrak{D}\right)$. As $i \rightarrow \infty$ we have $\rho_{\xi^{i}, \eta^{i}}(h) \rightarrow \rho_{0}(h)$. Hence if $U$ is any neighborhood of the fixed point $\zeta$ of $\rho_{0}(h)$ in $\overline{\mathbf{H}}$, the endpoints of the line $\widetilde{f}^{i}(\widetilde{C})$ lie in $U$ for all sufficiently large $i$. Hence for large $i$ we have $\widetilde{f}^{i}(\widetilde{C}) \subset U$. This implies the first assertion of the lemma.

To prove the second assertion we observe that since the geodesic map $\tilde{f}^{i} \mid \widetilde{C}$ is $F_{\widetilde{C}^{-}}$ equivariant, it is non-constant and is therefore a homeomorphism. This shows that for every $i$ we have $\widetilde{f}^{i}(z) \neq \widetilde{f}^{i}\left(z^{\prime}\right)$, and that $\widetilde{f}^{i}(\widetilde{C})$ is the unique line joining $\widetilde{f}^{i}(z)$ to $\widetilde{f}^{i}\left(z^{\prime}\right)$. 
Since we have shown that $\tilde{f}^{i}(\widetilde{C}) \subset U$ for large $i$, the second assertion follows.

8.23. Lemma. Let $\left(\xi^{i}, \eta^{i}\right)_{i \geq 1}$ be a sequence of points in $\mathfrak{A}$ which converges in $\mathfrak{D}$ to $(\alpha, \beta)$. For each $i \geq 1$ let $\tilde{f}^{i}: \Sigma \rightarrow \mathbf{H}^{3}$ be a $\left(\xi^{i}, \eta^{i}\right)$-good map, and let $f^{i}: \Sigma \rightarrow M\left(\left\langle\xi^{i}, \eta^{i}\right\rangle\right)$ be the map induced by $\tilde{f}^{i}$. Let $\epsilon$ be any positive number. Then for all sufficiently large $i$ the triple $\left(\Sigma, f^{i}, S\right)$ is an $\epsilon$-girded surface.

Proof. We must show that for sufficiently large $i$ the hyperbolically simplicial surface $\left(\Sigma, f^{i}\right)$ is non-degenerate and conditions (i) - (iv) of the definition 7.1 of an $\epsilon$-girded surface hold. Conditions (i) and (ii) are properties of the virtually triangulated surface $\Sigma$ and the 1-manifold $S \subset \Sigma$ that have been established in 8.17 and 8.19. We claim that condition (iv) holds for every $i$. Indeed, the isomorphisms $\rho_{0}: F \rightarrow\langle\alpha, \beta\rangle$ and $\rho_{\left(\xi^{i}, \eta^{i}\right)}: F \rightarrow\left\langle\xi^{i}, \eta^{i}\right\rangle$ give rise to an isomorphism (well-defined modulo inner automorphisms) between $\langle\alpha, \beta\rangle=\pi_{1}(M(\langle\alpha, \beta\rangle))$ and $\left\langle\xi^{i}, \eta^{i}\right\rangle=\pi_{1}\left(M\left(\left\langle\xi^{i}, \eta^{i}\right\rangle\right)\right)$. If we identify $\pi_{1}(M(\langle\alpha, \beta\rangle))$ and $\pi_{1}\left(M\left(\left\langle\xi^{i}, \eta^{i}\right\rangle\right)\right)$ via this isomorphism, then the equivariance condition in the definition of a good map implies that $f_{\#}: \pi_{1}(\Sigma) \rightarrow \pi_{1}\left(M\left(\left\langle\xi^{i}, \eta\right\rangle^{i}\right)\right)$ is identified (modulo inner automorphisms) with the inclusion homomorphism $\pi_{1}(\Sigma) \rightarrow \pi_{1}(M(\langle\alpha, \beta\rangle)$. Our claim therefore follows from Lemma 8.17.

Next we show that condition (iii) in the definition of an $\epsilon$-girded surface holds for large $i$. It follows from the definition of a $(\xi, \eta)$-good map that for every $i$ the map $f^{i} \mid S$ is geodesic. Now let $S$ be any component of $S$. Then $S$ is a core curve of some component $A$ of $\mathcal{A}$. Choose any component $\widetilde{A}$ of $p_{0}^{-1}(A)$, and let $F_{\widetilde{A}}$ denote the stabilizer of $\widetilde{A}$ in $F$. Then $F_{\widetilde{A}}$ is also the stabilizer of the component $\widetilde{C}$ of $\widetilde{S}$ contained in $\widetilde{A}$. By $8.14, F_{\widetilde{A}}$ is cyclic and is generated by an element $h$ such that $\rho_{0}(h)$ is parabolic. Since $\left(\xi^{i}, \eta^{i}\right) \rightarrow(\alpha, \beta)$ as $i \rightarrow \infty$, it follows that trace $\rho_{\xi^{i}, \eta^{i}}(h) \rightarrow$ trace $\rho_{0}(h)= \pm 2$. Hence the translation length of $\rho_{\xi^{i}, \eta^{i}}(h)$ approaches 0 as $i \rightarrow \infty$. But the closed geodesic defined by the map $f^{i} \mid S$ is $\widetilde{C} / F_{\widetilde{A}}$. Hence the length of $f^{i} \mid S$ approaches 0 as $i \rightarrow \infty$, and is in particular $<\epsilon$ for large $i$.

It remains to show that $\left(\Sigma, f^{i}\right)$ is non-degenerate for large $i$. Recall from the definition (6.5) that for every virtual simplex $\varphi: \Delta \rightarrow \Sigma$ and every $i$, the map $f_{\varphi}^{i}=f^{i} \circ \varphi$ admits a continuous lift $\tilde{f}_{\varphi}^{i}: \Delta \rightarrow \mathbf{H}^{n}$ such that $\tilde{f}_{\varphi}^{i}(\Delta)$ is a hyperbolic simplex and either (i) $\operatorname{dim} \widetilde{f}_{\varphi}^{i}(\Delta)<\operatorname{dim} \Delta$ or (ii) $\tilde{f}_{\varphi}^{i}$ maps $\Delta$ homeomorphically onto $\widetilde{f}_{\varphi}^{i}(\Delta)$. To say that $\left(\Sigma, f^{i}\right)$ is non-degenerate for a given $i$ means that (ii) holds for every virtual simplex $\Delta$ of $\Sigma$. It is clearly enough to show this for the case of a virtual 2 -simplex. Since $\Delta$ has only finitely many simplices, it suffices to show that for each virtual 2 -simplex $\varphi$ there is an integer $i_{\varphi}$ such that (ii) holds for every $i \geq i_{\varphi}$.

Let $\widetilde{\varphi}$ be a virtual 2 -simplex in $\widetilde{\Sigma}$ that covers $\varphi$. Let $v_{j}(j=1,2,3)$ denote the vertices of $\widetilde{\varphi}$. If (i) holds for a given $i$ then the points $\widetilde{f}^{i}\left(v_{1}\right), \widetilde{f}^{i}\left(v_{2}\right)$ and $\tilde{f}^{i}\left(v_{3}\right)$ are collinear in $\mathbf{H}^{3}$. Thus we need only show that the $\widetilde{f}^{i}\left(v_{j}\right)$ are non-collinear for large $i$. According to conclusion (i) of Lemma 8.19, each $v_{j}$ lies in some component of $\widetilde{S}$. It follows from conclusion (iii) of Lemma 8.19 that at most two of the $v_{j}$ can lie in any given component of $\widetilde{S}$.

Consider first the case in which all the $v_{j}$ lie in three distinct components of $\widetilde{S}$. Let $\widetilde{C}_{j}$ denote the component of $\widetilde{S}$ containing $v_{j}$, and let $\widetilde{A}_{j}$ denote the component of $\widetilde{\mathcal{A}}$ 
containing $\widetilde{C}_{j}$. Then the horospheres $C\left(\widetilde{A}_{1}\right), C\left(\widetilde{A}_{2}\right)$ and $C\left(\widetilde{A}_{3}\right)$ are distinct components of $p_{0}^{-1}\left(M_{\left\{\epsilon_{0}\right\}}(\langle\alpha, \beta\rangle)\right)$. This implies that the base points $\zeta\left(\widetilde{A}_{j}\right)$ of the $C\left(\widetilde{A}_{j}\right)$ are three mutually distinct points in $S_{\infty}$. But by Lemma 8.22 we have for $j=1,2,3$ that $\tilde{f}^{i}\left(v_{j}\right) \rightarrow$ $\zeta\left(\widetilde{A}_{j}\right)$ as $i \rightarrow \infty$. It follows that the three points $\tilde{f}^{i}\left(v_{j}\right), j=1,2,3$, are non-collinear when $i$ is large.

There remains the case in which two of the $v_{j}$, say $v_{1}$ and $v_{2}$, lie in the same component $\widetilde{C}$ of $\widetilde{S}$, and the third, say $v_{3}$, lies in a component $\widetilde{C}^{\prime} \neq \widetilde{C}$ of $\widetilde{S}$. Let $\widetilde{A}$ and $\widetilde{A}^{\prime}$ denote the components of $\widetilde{\mathcal{A}}$ containing $\widetilde{C}$ and $\widetilde{C}^{\prime}$ respectively. Then $\zeta(\widetilde{A})$ and $\zeta\left(\widetilde{A}^{\prime}\right)$ are distinct points of $S_{\infty}$ and hence have disjoint neighborhoods $U$ and $U^{\prime}$ in $\overline{\mathbf{H}}$. By the first assertion of Lemma 8.22 we have $\tilde{f}^{i}\left(v_{3}\right) \in U^{\prime}$ for large $i$. On the other hand, the second assertion of Lemma 8.22 implies that $\widetilde{f}^{i}\left(v_{1}\right) \neq \widetilde{f}^{i}\left(v_{2}\right)$ for all $i$, and that for large $i$ the line joining $\widetilde{f}^{i}\left(v_{1}\right)$ to $\tilde{f}^{i}\left(v_{2}\right)$ is contained in $U$. Since $U \cap U^{\prime}=\emptyset$ it follows that the $\tilde{f}^{i}\left(v_{j}\right)$ are non-collinear for large $i$.

8.24. Lemma. Let $(\xi, \eta)$ be any point of $\mathfrak{A}$, let $\widetilde{f}$ be any $(\xi, \eta)$-good map and let $f: \Sigma \rightarrow$ $M(\langle\xi, \eta\rangle)$ be the map induced by $\widetilde{f}$. Let $L$ be any component of Fix $T_{\xi, \eta}$. Then Fix $T_{\Sigma} \subset \Sigma$ contains exactly two points, say $P_{+}$and $P_{-}$, which are mapped into $L$ by $f$. Furthermore, the arc in $L$ with endpoints $f\left(P_{+}\right)$and $f\left(P_{-}\right)$is enclosed by the singular surface $(\Sigma, f)$.

Proof. To prove the first assertion we use the notation of 8.5: thus the components of Fix $T_{\xi, \eta}$ are $L_{j}(\xi, \eta)$ for $j=1,2,3$. We must show that for each $j$ there are exactly two of the six fixed points of Fix $T_{\Sigma}$ that are mapped by $f$ into $L_{j}(\xi, \eta)$. The equivariance condition in the definition of a good map shows that $\widetilde{f}\left(\ell_{j}(\alpha, \beta)\right)=\widetilde{f}\left(\operatorname{Fix} \tau_{\alpha, \beta, j}\right) \subset \operatorname{Fix} \tau_{\xi, \eta, j}=\ell_{j}(\xi, \eta)$. Hence $f\left(L_{j}(\alpha, \beta)\right) \subset L_{j}(\xi, \eta)$. In particular $f$ maps $L_{j}(\alpha, \beta) \cap \Sigma$ into $L_{j}(\xi, \eta)$. But $L_{j}(\alpha, \beta) \cap \Sigma$ consists of fixed points of $T_{0}$, and has cardinality 2 by Lemma 8.15. Thus the first assertion is proved. Since $T_{(\xi, \eta)} \circ f=f \circ T_{\Sigma}$ by 8.20 , the second assertion follows from the first by virtue of Proposition 6.10.

Proof of Proposition 8.2. It suffices to prove that if $\epsilon$ is a positive number, and $\left(\xi^{i}, \eta^{i}\right)$ is a sequence of points in $\mathfrak{A}$ which converges to $(\alpha, \beta)$, then for every sufficiently large $i$ there exists an $\epsilon$-girded surface in $M(\langle\xi, \eta\rangle)$ which encloses at least one of the reference points of $M(\langle\xi, \eta\rangle)$. According to Lemma 8.21, for each $i$ there exists a $\left(\xi^{i}, \eta^{i}\right)$-good map $\widetilde{f}^{i}: \widetilde{\Sigma} \rightarrow \mathbf{H}^{3}$. Let $f^{i}: \Sigma \rightarrow M(\langle\alpha, \beta\rangle)$ denote the map induced by $\widetilde{f}^{i}$. By Lemma 8.23 , $\left(\Sigma, f^{i}, S\right)$ is an $\epsilon$-girded surface for every sufficiently large $i$. Thus in order to prove the proposition it suffices to show that for every sufficiently large $i$ the hyperbolically simplicial surface $\left(\Sigma, f^{i}\right)$ encloses some reference point of $M\left(\left\langle\xi^{i}, \eta^{i}\right\rangle\right)$.

Recall from 8.17 that $S$ has at least two non-separating components; by Lemma 8.18, each such component contains at least two fixed points of $T_{\Sigma}$. Thus card $\left(S \cap \operatorname{Fix} T_{\Sigma}\right) \geq 4$. On the other hand, we have Fix $T_{\Sigma} \subset$ Fix $T_{0}$, and $T_{0}$ has three components. Hence there must be two points $P_{+}, P_{-} \in S \cap \operatorname{Fix} T_{\Sigma}$ that lie in the same component $L$ of $\operatorname{Fix} T_{0}$. By Lemma 8.15 we must have $L \cap \widetilde{\Sigma}=\left\{P_{+}, P_{-}\right\}$. In the notation of 8.5 , we have $L=L_{j_{0}}(\xi, \eta)$ for some $j_{0} \in\{1,2,3\}$. Let us set $\ell=\ell_{j_{0}}(\alpha, \beta)$, so that $L=p_{0}(\ell)$. Then $\widetilde{\Sigma}$ meets $\ell$ in two points $\widetilde{P}_{+} \in p_{0}^{-1}\left(P_{+}\right)$and $\widetilde{P}_{-} \in p_{0}^{-1}\left(P_{+}\right)$.

By 8.5, $\ell$ is the fixed line of $\tau_{\alpha, \beta, j_{0}}=\widehat{\rho}_{0}\left(t_{j_{0}}\right)$. For each $i \geq 1$, we let $\ell^{i}$ denote the fixed 
line of $\tau_{\xi^{i}, \eta^{i}, j_{0}}=\widehat{\rho}_{\xi^{i}, \eta^{i}}\left(t_{j_{0}}\right)$, and set $L^{i}=p_{\xi^{i}, \eta^{i}}\left(\ell^{i}\right)$. Since $\widetilde{f}^{i}$ is $\widehat{F}$-equivariant for every $i$, we have $\widetilde{f}^{i}\left(\widetilde{P}_{-}\right), \widetilde{f}^{i}\left(\widetilde{P}_{+}^{i}\right) \in \ell^{i}$. Hence $f^{i}\left(P_{-}\right), f^{i}\left(P_{+}\right) \in L^{i}$. If $\alpha^{i}$ denotes the arc in $L^{i}$ with endpoints $f^{i}\left(P_{-}\right), f^{i}\left(P_{+}\right)$, it follows from Lemma 8.24 that $\left(\Sigma, f^{i}\right)$ encloses $\alpha^{i}$. We shall complete the proof of the proposition by showing that $\alpha^{i}$ contains a reference point of $M\left(\left\langle\xi^{i}, \eta^{i}\right\rangle\right)$ for sufficiently large $i$.

We have $\widetilde{P}_{-}, \widetilde{P}_{+} \in \widetilde{S} \subset \widetilde{\mathcal{A}}$. Let $\widetilde{C}_{+} \subset \widetilde{A}_{+}$and $\widetilde{C}_{-} \subset \widetilde{A}_{-}$denote the components of $\widetilde{S}$ and $\widetilde{\mathcal{A}}$ containing $\widetilde{P}_{+}$and $\widetilde{P}_{-}$respectively. Since $\widetilde{\mathcal{A}}=p_{0}^{-1}\left(N_{\left\{\epsilon_{0}\right\}}(\langle\alpha, \beta\rangle)\right)$ is invariant under the action of $\widehat{F}$ on $\mathbf{H}^{3}$ defined by the representation $\widehat{\rho}_{0}$, and since the involution $\tau_{\alpha, \beta, j_{0}}$ fixes $\widetilde{P}_{-}$and $\widetilde{P}_{+}$, the sets $\widetilde{A}_{-}$and $\widetilde{A}_{+}$are invariant under $\tau_{\alpha, \beta, j_{0}}$. Hence $\zeta\left(\widetilde{A}_{ \pm}\right)$, which is the base point of the unique horoball $C\left(\widetilde{A}_{ \pm}\right)$containing $\widetilde{A}_{ \pm}$, is fixed by $\tau_{\alpha, \beta, j_{0}}$. It follows that each of the points $\zeta\left(\widetilde{A}_{-}\right)$and $\zeta\left(\widetilde{A}_{+}\right)$is an endpoint of the line $\ell$. We shall show that $\zeta\left(\widetilde{A}_{-}\right) \neq \zeta\left(\widetilde{A}_{+}\right)$, so that $\zeta\left(\widetilde{A}_{-}\right)$and $\zeta\left(\widetilde{A}_{+}\right)$are the two endpoints of $\ell$.

Since the horospheres $C\left(\widetilde{A}_{-}\right)$and $C\left(\widetilde{A}_{+}\right)$contain the endpoints of $\ell$, they meet $\ell$ orthogonally; hence each of these horospheres meets $\ell$ in at most a single point. Since $P_{-} \neq P_{+}$, it follows that $C\left(\widetilde{A}_{-}\right) \neq C\left(\widetilde{A}_{+}\right)$. Thus the horospheres $C\left(\widetilde{A}_{-}\right)$and $C\left(\widetilde{A}_{+}\right)$are distinct components of $p_{0}^{-1}\left(M_{\left\{\epsilon_{0}\right\}}(\langle\alpha, \beta\rangle)\right)$. This implies that the base points $\zeta\left(\widetilde{A}_{-}\right)$and $\zeta\left(\widetilde{A}_{+}\right)$ of $C\left(\widetilde{A}_{-}\right)$and $C\left(\widetilde{A}_{+}\right)$are distinct. Thus $\zeta\left(\widetilde{A}_{-}\right)$and $\zeta\left(\widetilde{A}_{+}\right)$are indeed the two endpoints of $\ell$. For each $i \geq 1$, let $\widetilde{P}^{i}$ denote the point of $\ell^{i}$ that is closest to $w_{0}$. By definition, $P^{i}=p_{\xi} i_{\eta} i\left(\widetilde{P}^{i}\right)$ is a reference point of $M\left(\left\langle\xi_{i}, \eta_{i}\right\rangle\right)$. Let $\widetilde{P}$ denote the point of $\ell$ that is closest to $w_{0}$. By 1.9 we have $\ell^{i} \rightarrow \ell$ as $i \rightarrow \infty$, so that $\widetilde{P}^{i} \rightarrow \widetilde{P}$. On the other hand, since $\widetilde{P}_{+} \in \widetilde{S}_{+}$and $\widetilde{P}_{-} \in \widetilde{S}_{-}$, Lemma 8.22 gives that $\widetilde{f}^{i}\left(\widetilde{P}_{+}\right) \rightarrow \zeta\left(\widetilde{A}_{+}\right)$and $\widetilde{f}^{i}\left(\widetilde{P}_{-}\right) \rightarrow \zeta\left(\widetilde{A}_{-}\right)$ in $\overline{\mathbf{H}}$ as $i \rightarrow \infty$. Thus we have a sequence of triples of points $\left(\widetilde{f}^{i}\left(\widetilde{P}_{-}\right), \widetilde{P}^{i}, \widetilde{f}^{i}\left(\widetilde{P}_{+}\right)\right)_{i \geq 1}$; the points in the $i$-th triple all lie in the line $\ell_{i}$; and in the limit triple $\left(\zeta\left(\widetilde{A}_{-}\right), \widetilde{P}, \zeta\left(\widetilde{A}_{+}\right)\right)$, the point $\widetilde{P}$ lies on the line $\ell \subset \mathbf{H}^{3}$, whereas $\zeta\left(\widetilde{A}_{-}\right)$and $\zeta\left(\widetilde{A}_{+}\right)$are the endpoints of $\ell$. It follows that for sufficiently large $i$ the point $\widetilde{P}^{i}$ lies between $\widetilde{f}^{i}\left(\widetilde{P}_{-}\right)$and $\left.\widetilde{f}^{i}\left(\widetilde{P}_{+}\right)\right)$.

By Proposition 8.6 this means that for sufficiently large $i$ the point $P^{i}$ lies between $f^{i}\left(P_{-}\right)$and $\left.f^{i}\left(P_{+}\right)\right)$, so that $P^{i} \in \alpha^{i}$ for large $i$, as required.

\section{§9. Estimates FOR GEOMETRICALly Finite GROUPS}

9.1. The following theorem is the main result of this paper.

Theorem. Let $\xi$ and $\eta$ be non-commuting isometries of $\mathbf{H}^{3}$. Suppose that $\xi$ and $\eta$ generate a torsion-free discrete group which is topologically tame, is not co-compact and contains no parabolics. Then for any $z \in \mathbf{H}^{3}$ we have

$$
\max (\operatorname{dist}(z, \xi \cdot z), \operatorname{dist}(z, \eta \cdot z)) \geq \log 3
$$

The following two results are used in the proof of Theorem 9.1, and put the statement in perspective.

9.2. Proposition. Let $\xi$ and $\eta$ be non-commuting isometries of $\mathbf{H}^{3}$ that generate a torsion-free discrete group which is not co-compact and contains no parabolics. Then $\langle\xi, \eta\rangle$ is a free group on the generators $\xi$ and $\eta$. 
Proof. Set $\Gamma=\langle\xi, \eta\rangle$. It follows from the hypotheses that the space $M=\mathbf{H}^{3} / \Gamma$ is a complete, non-compact, orientable hyperbolic 3-manifold without cusps. In particular, the universal cover of $M$ is homeomorphic to $\mathbf{R}^{3}$, so that $M$ is irreducible; and $\Gamma=\pi_{1}(M)$ has no free abelian subgroup of rank 2 .

It follows from [BaS, Proposition 3 and Theorem $\mathrm{A}$ ] that if $M$ is any non-compact, irreducible, orientable 3-manifold such that $\pi_{1}(M)$ has no free abelian subgroup of rank 2, then $\pi_{1}(M)$ is 2-free in the sense that each of its 2-generator subgroups is free of some rank $\leq 2$. (This fact is also implicit in [Tuc] and [JaS, Sect. VI.4].) Thus $\Gamma$ is 2-free; since $\Gamma$ is itself generated by two non-commuting elements, it is a free group of rank 2 . Hence by $[\mathrm{K}, \mathrm{p} .59], \Gamma$ is in fact free on the given generators $\xi$ and $\eta$.

9.3. Proposition. Suppose that $\xi$ and $\eta$ satisfy the hypotheses of Theorem 9.1. Then either the group $\langle\xi, \eta\rangle$ is geometrically finite, or we have $M(\langle\xi, \eta\rangle)=N(\langle\xi, \eta\rangle)$.

Proof. Since $\Gamma=\langle\xi, \eta\rangle$ is not co-compact, it follows from Proposition 9.2 that $\Gamma$ is a free group. In particular, $H_{2}(\Gamma ; \mathbf{Z})=0$.

According to 1.4 , the set $N=N(\Gamma) \subset M(\Gamma)$ is a 3-manifold-with-boundary and a deformation retract of $M(\Gamma)$. Thus $N$ is aspherical and $\pi_{1}(N) \cong \Gamma$, and therefore $H_{2}(N ; \mathbf{Z}) \cong H_{2}(\Gamma ; \mathbf{Z})=0$. If $\partial N$ has a compact component, it follows that $N$ is compact (and has connected boundary); by the definition (1.7) this implies that $\Gamma$ is geometrically finite. On the other hand, if $\partial N=\emptyset$ then $N=M(\Gamma)$. Hence to prove the proposition it suffices to show that $\partial N$ is compact.

Let $\Omega$ denote the set of discontinuity of $\Gamma$. The surface $\Omega / \Gamma$ inherits a conformal structure from $\Omega$. According to the Ahlfors finiteness theorem $[\mathrm{A}], \Omega / \Gamma$ is a hyperbolic Riemann surface which has finite area in the Poincaré metric. Since $\Gamma$ contains no parabolics, it also follows from Ahlfors's theorem that $\Omega / \Gamma$ has no cusps. Hence $\Omega / \Gamma$ is compact. We shall prove that $\partial N$ is compact by showing that there is a continuous map of $\Omega / \Gamma$ onto $\partial N$. For this purpose it suffices to produce a $\Gamma$-equivariant surjection $\Theta: \Omega \rightarrow \partial \widetilde{N}$, where $\widetilde{N}=\operatorname{nbhd}_{1}\left(\operatorname{hull}\left(\Lambda_{\Gamma}\right)\right)$ is the pre-image of $N$ in $\mathbf{H}^{3}$. Recall from 1.4 that $\widetilde{N}$ is convex.

For any point $\zeta \in \Omega$ let $C_{\zeta}$ denote the smallest horoball based at $\zeta$ which meets the set $\widetilde{N}$. Then $C_{\zeta}$ meets $\widetilde{N}$ in a single point; for if $z$ and $z^{\prime}$ were distinct points of $\widetilde{N} \cap C_{\zeta}$ then, since a horoball is convex, the open line segment in $\mathbf{H}^{3}$ joining $z$ to $z^{\prime}$ would be contained in $\widetilde{N} \cap$ int $C_{\zeta}$, and the minimality of $C_{\zeta}$ would be contradicted. We define $\Theta(\zeta)$ to be the point of intersection of $C_{\zeta}$ with $\widetilde{N}$. It is clear that $\Theta(\zeta) \in \partial \widetilde{N} \cap \partial C_{\zeta}$ for each $\zeta \in \Omega$.

The function $\Theta: \Omega \rightarrow \partial \widetilde{N}$ is obviously $\Gamma$-equivariant. To see that it is surjective, we observe that since $\widetilde{N}$ is closed and convex, every point $z \in \partial \widetilde{N}$ lies on the boundary of a hyperbolic half-space $Q \subset \mathbf{H}^{3}$ with $\widetilde{N} \cap \operatorname{int} Q=\emptyset$. (One deduces the existence of such a "supporting half-space" for a closed convex set in $\mathbf{H}^{3}$ from the corresponding fact in Euclidean space by using the projective model as in 1.2.) Now let $C$ denote the unique horoball which is contained in $Q$ and tangent to the plane $\partial Q$, and let $\zeta$ denote the base point of $C$. It is clear that $\zeta \in \Omega$ and that $\Theta(\zeta)=z$. Thus $\Theta$ is surjective.

It remains to show that $\Theta$ is continuous. For this purpose we identify $\mathbf{H}^{3}$ with the upper half-space model $\mathbf{R}^{2} \times(0, \infty)$ by an isometry that maps some point of $\Lambda_{\Gamma}$ to $\infty$. This guarantees that every vertical ray in $\mathbf{R}^{2} \times(0, \infty)$ whose lowest point is in $N$ is entirely contained in $N$. Now let $\left(\zeta_{i}\right)$ be a convergent sequence in $\Omega$ with $\zeta_{\infty}=\lim \zeta_{i} \in \Omega$. For 
$1 \leq i \leq \infty$ we set $z_{i}=\Theta\left(\zeta_{i}\right)$ and $C_{i}=C_{\zeta_{i}}$. The closure $\overline{C_{i}}$ of $C_{i}$ in $\mathbf{R}^{2} \times[0, \infty)$ is a Euclidean ball of some radius $r_{i}$, tangent to $\mathbf{R}^{2} \times\{0\}$ at $\zeta_{i}$, and $\overline{C_{i}} \cap N=\left\{z_{i}\right\}$. Since the vertical ray with lowest point $z_{i}$ is contained in $N$, the point $z_{i}$ must lie on the closed upper hemisphere $U_{i}$ of the sphere $\partial \overline{C_{i}}$.

We claim that $r_{i} \rightarrow r_{\infty}$ as $i \rightarrow \infty$. If this is false then after passing to a subsequence we can assume that $r_{i} \rightarrow r$ for some $r \neq r_{\infty}$ in $[0, \infty]$. If $r>r_{\infty}$ then for large enough $i$ we have $U_{\infty} \subset \operatorname{int} C_{i}$, and in particular $z_{\infty} \in \operatorname{int} C_{i}$. This is impossible since $\overline{C_{i}} \cap N=$ $\left\{z_{i}\right\} \subset \partial \overline{C_{i}}$. Similarly, if $r<r_{\infty}$ then for large $i$ we have $U_{i} \subset \operatorname{int} C_{\infty}$, and again we obtain a contradiction. This proves the claim.

Now suppose that $\left(z_{i}\right)$ does not converge to $z_{\infty}$. Then after passing to a subsequence we may assume that $z_{i} \rightarrow w$ for some point $z \in N-\left\{z_{\infty}\right\}$. Since $z_{i} \in \overline{C_{i}}$ for $1 \leq i<\infty$, and since $\zeta_{i} \rightarrow \zeta$ and $r_{i} \rightarrow r$, we have $z \in \overline{C_{\infty}}$. This is impossible since $\overline{C_{\infty}} \cap N=\left\{z_{\infty}\right\}$. Hence we must have $z_{i} \rightarrow z_{\infty}$. This shows that $\Theta$ is continuous and completes the proof.

9.4. By Proposition 9.3, the proof of Theorem 9.1 breaks up into two disjoint cases, according to whether the group $\Gamma=\langle\xi, \eta\rangle$-which is free by 9.2 - satisfies $N(\Gamma)=M(\Gamma)$ or is geometrically finite. The first case is covered by the following result.

Lemma. Let $\xi$ and $\eta$ be non-commuting isometries of $\mathbf{H}^{3}$ that freely generate a rank-2 free Kleinian group $\Gamma$ which is topologically tame, contains no parabolics and satisfies $N(\Gamma)=M(\Gamma)$. Then for any $z \in \mathbf{H}^{3}$ we have

$$
\max (\operatorname{dist}(z, \xi \cdot z), \operatorname{dist}(z, \eta \cdot z)) \geq \log 3
$$

Proof. According to Proposition 6.9 every positive superharmonic function on the manifold $M(\Gamma)$ is constant. Thus by Proposition 3.9, every $\Gamma$-invariant conformal density is a constant multiple of the area density. By Proposition 5.2 we therefore have max ( $\operatorname{dist}(z, \xi$. $z), \operatorname{dist}(z, \eta \cdot z)) \geq \log 3$ for every $z \in \mathbf{H}^{3}$.

9.5. The following result covers the remaining case of Theorem 9.1.

Lemma. Let $\xi$ and $\eta$ be non-commuting elements in $\mathrm{PSL}_{2}(\mathbf{C})$ that freely generate a rank2 free Kleinian group which is geometrically finite and contains no parabolics. Then for any $z \in \mathbf{H}^{3}$ we have

$$
\max (\operatorname{dist}(z, \xi \cdot z), \operatorname{dist}(z, \eta \cdot z)) \geq \log 3
$$

Proof. We use the notation of Section 8. Let a point $z \in \mathbf{H}^{3}$ be given. Let us define a continuous non-negative-valued function $f$ on $V$ by

$$
f(\xi, \eta)=\max (\operatorname{dist}(z, \xi \cdot z), \operatorname{dist}(z, \eta \cdot z))
$$

The proposition asserts that $f(\xi, \eta) \geq \log 3$ for all $(\xi, \eta) \in \mathfrak{G} \mathfrak{F}$; or, equivalently, that $f(\xi, \eta) \geq \log 3$ for all $(\xi, \eta) \in \overline{\mathfrak{G} \mathfrak{F}}$.

Every local minimum of the function $f$ on $V$ occurs at a point $(\xi, \eta)$ such that either $\xi$ or $\eta$ fixes $z$. Indeed, if $(\xi, \eta)$ is a point of $V$ where $\xi(z) \neq z$ and $\eta(z) \neq z$, then there exist a sequence $\left(z_{i}\right)$ of points on the open hyperbolic line segment between $z$ and $\xi(z)$ which 
converges to $\xi(z)$, and a sequence $\left(w_{i}\right)$ of points on the open segment between $z$ and $\eta(z)$ which converges to $\eta(z)$. If we choose sequences $\left(\alpha_{i}\right)$ and $\left(\beta_{i}\right)$ of hyperbolic isometries converging to the identity, such that $\alpha_{i}(z)=z_{i}$ and $\beta_{i}(z)=w_{i}$, then $\left(\alpha_{i} \xi, \beta_{i} \eta\right)$ converges to $(\xi, \eta)$ as $i \rightarrow \infty$, and $f\left(\alpha_{i} \xi, \beta_{i} \eta\right)<f(\xi, \eta)$ for all $i$. Hence $(\xi, \eta)$ is not a local minimum of $f$.

For any point $(\xi, \eta) \in \mathfrak{D}$, the elements $\xi$ and $\eta$ of $\operatorname{PSL}_{2}(\mathbf{C})$ have infinite order in the discrete group $\langle\xi, \eta\rangle$, and hence have no fixed points in $\mathbf{H}^{3}$. It follows that $f$ has no local minimum on the open subset $\mathfrak{G} \mathfrak{F}$ of $V$.

On the other hand, $f$ is a proper function on $V$; indeed, for any $B \geq 0$, the set $f^{-1}([0, B])$ consists of all pairs $(\xi, \eta)$ such that $\xi$ and $\eta$ each move the point $z$ a distance at most $B$, and this set is clearly compact. Hence $f$ must take a minimum value on the closed set $\overline{\mathfrak{G} \mathfrak{F}}$. Since it has no local minimum on the open set $\mathfrak{G} \mathfrak{F}$, it must take its minimum value on $\mathfrak{B}$. Hence we need only show that $f(\xi, \eta) \geq \log 3$ for all $(\xi, \eta) \in \mathfrak{B}$.

Let $\mathfrak{C} \subset \mathfrak{B}$ denote the dense $G_{\delta}$ given by Theorem 8.2. Since $f$ is continuous and $\mathfrak{C}$ is dense in $\mathfrak{B}$, we need only prove that $f(\xi, \eta) \geq \log 3$ for all $(\xi, \eta) \in \mathfrak{C}$. But for any $(\xi, \eta) \in \mathfrak{C}$, the Kleinian group $\langle\xi, \eta\rangle$ is girded; hence by Proposition 7.4 , every positive superharmonic function on the manifold $M(\Gamma)$ is constant. Thus according to Proposition 3.9 , every $\langle\xi, \eta\rangle$-invariant conformal density is a constant multiple of the area density. By Proposition 5.2 we therefore have

$$
f(\xi, \eta)=\max (\operatorname{dist}(z, \xi \cdot z), \operatorname{dist}(z, \eta \cdot z)) \geq \log 3
$$

Proof of Theorem 9.1. The theorem follows immediately from Propositions 9.2 and 9.3 and Lemmas 9.4 and 9.5.

\section{§10. Applications to ClOSED hyperbolic MANifolds}

10.1. The following result is an immediate consequence of Theorem 9.1 and the definition of a Margulis number given in Section 1.

Proposition. Let $M=\mathbf{H}^{3} / \Gamma$ be a closed, orientable hyperbolic 3-manifold such that every 2-generator subgroup of $\Gamma=\pi_{1}(M)$ has non-empty set of discontinuity in $S_{\infty}$. Then $\log 3$ is a Margulis number for $M$.

10.2. In this section we shall prove the

Proposition. Let $M$ be a closed, orientable hyperbolic 3-manifold such that the rank of $H_{1}(M ; \mathbf{Q})$ is at least 3. Then every 2-generator subgroup of $\pi_{1}(M)$ is non-co-compact and topologically tame.

10.3. By combining Propositions 10.1 and 10.2 one immediately obtains the following result.

Theorem. Let $M$ be a closed, orientable hyperbolic 3-manifold such that the rank of $H_{1}(M ; \mathbf{Q})$ is at least 3. Then $\log 3$ is a Margulis number for $M$. 
10.4. Corollary. Let $M$ be a closed, orientable hyperbolic 3-manifold such that the rank of $H_{1}(M ; \mathbf{Q})$ is at least 3. Then $M$ contains an isometric copy of a ball of radius $\frac{1}{2} \log 3$ in $\mathbf{H}^{3}$. Hence the volume of $M$ is greater than 0.92 .

Proof. By Theorem 10.3, the number $\epsilon=\log 3$ is a Margulis number for $M$. Thus $M_{(0, \epsilon]}$ is a disjoint union of tubes in $M$, and in particular $M_{(\epsilon, \infty]} \neq \emptyset$. It follows from the definition of $M_{(\epsilon, \infty]}$ that the $(\epsilon / 2)$-neighborhood of any point of $M_{(\epsilon, \infty]}$ is isometric to a ball of radius $\epsilon / 2$ in $\mathbf{H}^{3}$. This proves the first assertion.

To deduce the second assertion from the first, we use an observation due to Meyerhoff [Me]. Using a result due to Böröczky [Bör], Meyerhoff shows that if a hyperbolic manifold $M$ contains a hyperbolic ball $B(r)$ of a given radius $r$ then the volume of $M$ is at least $2 \operatorname{Vol}(T) /(6 \alpha-\pi)$, where $\alpha=\frac{1}{2} \operatorname{arcsec}(2+\operatorname{sech} 2 r)$, and $T$ is a regular tetrahedron in $\mathbf{H}^{3}$ whose edges have length $2 r$. (This is equivalent to the inequality $\operatorname{Vol}(M) \geq \operatorname{Vol}(B(r)) / d(r)$ given on p. 277 of [Me]: the factor $\sinh (2 r)-2 r$ in the definition of $d(r)$ on p. 275 of [Me] is equal by [F, p. 209] to $\frac{1}{2} \operatorname{Vol}(B(r))$.) Taking $r=\frac{1}{2} \log 3$, and using Lobachevsky functions as in $[F$, Sect. IX.4] to compute the volume of a regular hyperbolic tetrahedron with edges of length $\log 3$, one obtains the estimate $\operatorname{Vol} M>0.92$.

Proof of Proposition 10.2. Let $F$ be any 2-generator subgroup of $\Gamma=\pi_{1}(M)$. Since $H_{1}(M ; \mathbf{Q})$ has rank at least 3, there is an epimorphism $\eta: \pi_{1}(M) \rightarrow \mathbf{Z}$ such that $\eta(F)=$ $\{0\}$. In particular, $F$ has infinite index in $\Gamma$ and is therefore non-co-compact. It remains to show that $F$ is topologically tame.

It follows from [He, Lemma 6.5] that $\eta$ is induced by some map $\phi: M \rightarrow S^{1}$ such that $\phi$ is transverse to $1 \in S^{1}$ and the surface $\phi^{-1}(1)$ is incompressible; that is, for each component $\Sigma$ of $\phi^{-1}(1)$, we have $\pi_{1}(\Sigma) \neq\{1\}$, and the inclusion homomorphism $\pi_{1}(\Sigma) \rightarrow \pi_{1}(M)$ is injective.

Let $\left(\widetilde{M}_{1}, p_{1}\right)$ denote the covering space of $M$ corresponding to the subgroup $\Gamma_{1}=\operatorname{ker} \eta$ of $\Gamma$. Then $\phi$ is covered by a map $\widetilde{\phi}$ from $\widetilde{M}_{1}$ to the universal cover $\mathbf{R}$ of $S^{1}$. Let $\mathcal{G}$ be a graph whose fundamental group is free of $\operatorname{rank} 2$, and let $\psi: \mathcal{G} \rightarrow M$ be a basepoint-preserving map such that $\psi_{\#}\left(\pi_{1}(\mathcal{G})\right)=F$. Since $F \subset \operatorname{ker} \eta$, the map $\psi$ admits a lift $\widetilde{\psi}: \mathcal{G} \rightarrow \widetilde{M}_{1}$. Since $\mathcal{G}$ is compact, we have $\widetilde{\psi}(\mathcal{G}) \subset \widetilde{\phi}^{-1}([-D, D])$ for some positive integer $D$. The component of $\widetilde{\phi}^{-1}([-D, D])$ containing $\widetilde{\psi}(\mathcal{G})$ is a compact 3-manifold-with-boundary $K_{1}$ with $\partial K_{1} \subset p_{1}^{-1}\left(\phi^{-1}(1)\right)$; hence $\partial K_{1}$ is incompressible in $\widetilde{M}_{1}$. We define $\Gamma_{2} \leq \Gamma_{1} \leq \Gamma$ to be the image of the inclusion homomorphism $\pi_{1}\left(K_{1}\right) \rightarrow \pi_{1}\left(\widetilde{M}_{1}\right)$, so that $F \leq \Gamma_{2}$.

Let $\left(\widetilde{M}_{2}, p_{2}\right)$ denote the covering space of $\widetilde{M}_{1}$ corresponding to the subgroup $\Gamma_{2}$. Then $\widetilde{M}_{2}$ contains a submanifold $K_{2}$ which is mapped homeomorphically onto $K_{1}$ by $p_{2}$. Thus if we denote the boundary components of $K_{2}$ by $\widetilde{\Sigma}^{1}, \ldots, \widetilde{\Sigma}^{k}$, each $\widetilde{\Sigma}^{j}$ is mapped homeomorphically by $p_{2}$ onto a component $\Sigma^{j}$ of $\partial K$. It is clear that $K_{2}$ is a compact core of $\widetilde{M}_{2}$ in the sense that the inclusion homomorphism $\pi_{1}\left(K_{2}\right) \rightarrow \pi_{1}\left(\widetilde{M}_{2}\right)$ is an isomorphism. It is also clear that the $\widetilde{\Sigma}^{j}$ are incompressible.

Thus $\widetilde{M}_{2}$ is a hyperbolic 3-manifold having a compact core whose boundary is incompressible. It follows from the main theorem of [Bo] that any such hyperbolic manifold is topologically tame. On the other hand, $\Gamma_{2}$ is contained in $\Gamma_{1}$ and therefore has infinite index in $\Gamma$; hence $\widetilde{M}_{2}$ has infinite volume. According to [Ca, Proposition 3.2], any cover- 
ing space, with finitely generated fundamental group, of an infinite-volume topologically tame hyperbolic 3-manifold is itself topologically tame. Since $F \leq \Gamma_{2}$, it follows that $F$ is topologically tame, as required.

A stronger version of Proposition 10.2 can be established: namely that under the same hypothesis, every 2-generator subgroup of $\pi_{1}(M)$ is in fact geometrically finite. This can be proved by combining results of [Th. Chapter 9] and [ScS]; see also [Ca2].

\section{REFERENCES}

[A] L. Ahlfors, Finitely generated Kleinian groups, Amer J. Math. 86 (1964), 413-429.

[BaS $]$ G. Baumslag and P. Shalen, Groups whose 3-generator subgroups are free, Bull. Austral. Math. Soc. 40 (1989), $163-174$.

[Be] A. F. Beardon, The geometry of discrete groups, Springer-Verlag, c. 1983.

[Bo] F. Bonahon, Bouts des variétés hyperboliques de dimension 3, Ann. of Math. 124 (1986), 71-158.

[Bör] K. Böröczky, Packing of spheres in spaces of constant curvature, Acta Math. Acad. Sci. Hungar. 32 (1978), 243- 261.

[Ca1] R. Canary, Ends of hyperbolic 3-manifolds, Pre-print.

[Ca2] R. Canary, Covering theorems for hyperbolic 3-manifolds, to appear.

[Ch] V. Chuckrow, On Schottky groups with applications to Kleinian groups, Ann. of Math 88 (1968), $47-61$.

[Co] H. S. M.Coxeter, Non-Euclidean Geometry, University of Toronto Press, 1947.

[CuS $]$ M. Culler and P. B. Shalen, Varieties of group representations and splittings of 3-manifolds, Ann. of Math 117 (1983), 109-146.

[F] W. Fenchel, Elementary Geometry in Hyperbolic Space, de Gruyter Studies in Mathematics, vol. 11, Walter de Gruyter, Berlin \& New York, 1989.

[GeM] F. Gehring and G. Martin, Inequalities for Möbius transformations and discrete groups, preprint.

[He] J. Hempel, 3-manifolds (1976), Princeton Univ. Press.

[JaS] W. H. Jaco and P. B. Shalen, Seifert fibered spaces in 3-manifolds, Memoirs Amer. Math. Soc. 21 (1979), no. 220.

[Joh] K. Johannson, Homotopy equivalences of 3-manifolds with boundaries, Lecture Notes in Mathematics, vol. 761, Springer-Verlag 1979.

[Jør] T. Jørgensen, Closed geodesics on Riemann surfaces, Proc. Amer. Math. Soc. 72 (1978), 140-142.

[K] A. G. Kurosh, Theory of groups, volume II, Chelsea, 1960.

[Mal] A. Malcev, On isomorphic matrix representations of infinite groups, Rec. Math. [ Math. Sbornik], N. S. 8 (50) (1940), 405-422.

[Mar] A. Marden, The geometry of finitely generated Kleinian groups, Ann. of Math. 99 (1974), 383462.

$[\mathrm{McM}]$ C. T. McMullen, Cusps are dense, Ann. of Math. (to appear).

[Me] R. Meyerhoff, Sphere-packing and volume in hyperbolic 3-space, Comm. Math. Helv. 61 (1986), 271-278.

[N] P. J. Nicholls, The ergodic theory of discrete groups, London Math. Soc. Lecture Noties Series, vol. 143, Cambridge Univ. Press, 1989.

[P] S. J. Patterson, Analytic and geometric aspects of hyperbolic space, Warwick and Durham, 1984 (D. B. A. Epstein ed.), London Math. Soc. Lecture Note Series, Cambridge University Press, 1987.

[ScS] G. P. Scott and G. A. Swarup, Geometric finiteness of certain Kleinian groups, Proc. Amer. Math. Soc. 109 (1990), 765-768.

[ShW] P. B. Shalen and P. Wagreich, Growth, volumes, and free subgroups of 3-manifold groups, Trans. Amer. Math. Soc. (to appear).

[Su1] D. Sullivan, The density at infinity of a discrete group of hyperbolic motions, Publ. Math. I.H.E.S. 50 (1979), 419-450. 
[Su2] - On the ergodic theory at infinity of an arbitrary discrete group of hyperbolic motions, Riemann surfaces and related topics: Proceedings of the 1978 Stony Brook Conference, Ann. of Math. Studies, vol. 97, Princeton Univ. Press, 1980, pp. 465-496.

[Su3] - Growth of positive harmonic functions and Kleinian group limit sets of zero planar measure and Hausdorff dimension 2, Geometry Symposium Utrecht 1980, Lecture Notes in Mathematics 894, Springer-Verlag 1981, pp. 127-144.

[Su4] - Quasiconformal homeomorphisms and dynamics II: Structural stability implies hyperbolicity for Kleinian groups, Acta Math. 155 (1984), 243-260.

[Th] W. Thurston, The geometry and topology of 3-manifolds, Photocopied notes, Princeton University, 1978.

[Tuc] T. Tucker, On Kleinian groups and 3-manifolds of Euler characteristic zero, Unpublished.

[Wag] S. Wagon, The Banach-Tarski paradox, Cambridge Univ. Press, 1985.

[Wal] F. Waldhausen, On irreducible 3-manifolds which are sufficiently large, Ann. of Math. 87 (1968), 56 - 88 . 